\title{
Coding and Non-Coding RNA in Age-Associated MEMORY IMPAIRMENT AND ALZHEIMER's DiseASE
}

\author{
PhD Thesis
}

in partial fulfillment of the requirements

for the degree "Doctor rerum naturalium"

in the Neuroscience Program

at the Georg-August-University Göttingen, Faculty of Biology

submitted by

Pooja Rao

born in

Madras / India

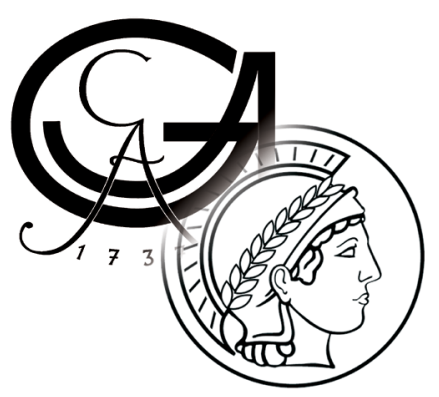

Göttingen 2013 



\section{PhD Thesis Committee:}

1. Prof. Dr. André Fischer: Deutsches Zentrum für Neurodegenerative Erkrankungen (DZNE), University Medical Center, Göttingen (Reviewer)

2. Dr. Wolfgang Fischle: Max Planck Institute for Biophysical Chemistry, Göttingen (Reviewer)

3. Dr. Judith Stegmüller: Max Planck Institute for Experimental Medicine, Göttingen

\section{Examination Committee:}

Members of the PhD thesis committee and

- $\quad$ Prof. Dr. Michael Hörner: Johann Friedrich Blumenbach Institute for Zoology and Anthropology and IMPRS Neurosciences, Georg-August-Universität, Göttingen

- Dr. Ira Milosevic: European Neuroscience Institute Göttingen (ENI-G), University Medical Center and Max-Planck Society, Göttingen

- $\quad$ Prof. Dr. Mikael Simons: Max-Planck-Institut for Experimental Medicine, and University Medical Center ,Göttingen

Date of Oral Examination: $\quad 15.01 .2014$ 



\section{Declaration}

I herewith declare that I have prepared the dissertation 'Coding and non-coding RNA in age-associated memory impairment and Alzheimer's disease' entirely by myself with no other aids or sources than quoted.

Göttingen, 09.12.13

Pooja Rao 



\section{Table of contents}

Acknowledgements 11

List of Figures $\quad 13$

$\begin{array}{ll}\text { List of abbreviations } & 15\end{array}$

1. Introduction

1.1 Learning, memory and age-associated memory impairment (AAMI) 19

1.1.1 The hippocampus in learning and memory and AAMI 19

1.1.2 Synaptic plasticity and transcription $\quad 21$

1.1.3 Brain gene expression changes in aging and Alzheimer's disease 22

1.1.4 MicroRNAs in learning and memory 23

1.1.5 Mouse models in the study of learning and memory 25

1.1.6 Genome-wide gene expression profiling as a method to study disease pathophysiology 26

1.2 Molecular Mechanisms of Alzheimer's disease (AD) 29

1.2.1 Alzheimer's disease: Causes and hypotheses 29

1.2.2 Age as a risk factor for AD — Parallels and contrasts between AAMI and AD 30

1.2.3 Need for new methods to study Alzheimer's disease 31

1.2.4 Current state of biomarkers in AD 32

1.2.5 Extracellular vesicles $\quad 34$

1.2.6 Exosomes and extracellular vesicles in CNS physiology and Pathology 34

1.3 Objectives

1.3.1 RNA in age-related memory impairment 41

1.3.2 RNA in Alzheimer's disease $\quad 41$

2. Methods and Materials

2.1 Animals and behavior experiments 45

2.1.1 Animal care $\quad 45$

2.1.2 Water maze Task $\quad 45$

2.1.3 Fear Conditioning Task $\quad 45$

2.1.4 Novel object recognition test 45

2.1.5 Rotarod test $\quad 45$

2.1.6 APPPS1-21 mice $\quad 46$

2.2 Cell culture and Exosomes $\quad 47$

$\begin{array}{ll}\text { 2.2.1 Primary neuron culture } & 47\end{array}$

2.2.4 Exosome Isolation $\quad 48$

2.3 Cerebrospinal fluid and plasma from patients 48

2.4 RNA Isolation and profiling $\quad 49$

2.4.1 RNA isolation from exosomes, tissues and cultured cells 49

2.4.2 cDNA synthesis and qPCR 49

2.5 Bioinformatics and statistical analysis $\quad 50$

2.5.1 Primary and secondary analysis of next-generation sequencing data 50 

2.5.2 Statistical and functional analysis of next-generation sequencing data

3. Results

3.1 Age-associated memory impairment 55

3.1.1 Aging is associated with increased variability in water maze task performance 55

3.1.2 No increase in variability on other learning tasks, visual or motor skills 55

3.1.3 Individual performance on behavior tests is consistent 58

3.1.4 Gene expression in aging mice $\quad 61$

3.1.5 Gene sets that correlate with memory performance 63

3.1.6 Over- and under-expressed pathways in high-performing aged mice 66

3.1.7 MicroRNA expression in aging mice $\quad 72$

3.2 Alzheimer's disease $\quad 74$

3.2.1 Gene and microRNA expression in the APP-PS1 mouse model $\quad 74$

$\begin{array}{ll}\text { 3.2.2 RNA isolation from human cerebrospinal fluid } & 76\end{array}$

3.2.3 The RNA contents of the CSF exosome $\quad 78$

3.2.4 Exosomal RNA versus cellular RNA $\quad 82$

3.2.5 Sequencing RNA from small volumes of CSF $\quad 85$

3.2.6 CSF RNA in Alzheimer's disease $\quad 85$

4. Discussion

4.1 Gene expression changes in age-associated memory impairment 95

4.1.1 Heterogeneity in aging $\quad 95$

4.1.2 Distinguishing aging individuals with age-associated memory impairment 96

4.1.3 Comparison with previous rodent studies on age-associated memory impairment 97

4.1.4 Ribosomes, translation and RNA-binding proteins in learning and memory 99

4.1.5 Glutamate receptors, excitotoxicity and memory impairment 99

4.2 RNA in the study of Alzheimer's disease $\quad 100$

4.2.1 Gene and microRNA expression in mouse models of brain amyloidosis $\quad 100$

4.2.2 Feasibility of RNA isolation from CSF 103

4.2.3 Choice of methods for RNA isolation from CSF 103

4.2.4 RNA species in CSF exosomes $\quad 104$

4.2.5 Exosomal RNA as an indicator of intracellular changes 106

$\begin{array}{ll}\text { 4.2.6 Potential for use of CSF RNA as a biomarker for AD } & 107\end{array}$

4.3 Conclusions

4.3.1 RNA in age-associated memory impairment 111

4.3.2 CSF exosomal RNA in the study of AD 111

$\begin{array}{ll}\text { References } & 115\end{array}$

$\begin{array}{ll}\text { Appendix 1: Notes on Gene set enrichment analysis } & 137\end{array}$

Appendix 2: Notes on the Random Forest machine learning algorithm and the Gini Index 139 



\section{Acknowledgements}

I would like to thank Andre for guiding me during this work, enabling my introduction to the wonderful world of next generation sequencing and for allowing me a great degree of freedom while being an inspiring mentor.

For their companionship during the past 3 years, I would like to thank all the members of the Fischer lab, past and present. In particular, I would like to acknowledge Susanne and Anna-Lena for help with the experiments, Torsten and Roberto for being great office-mates, Eva and Rashi for advice during the final phases, and Roman for helpful comments on the thesis.

I would like to thank Anja Schneider and the members of the Schneider lab for all the exosomes, which were critical to this work. Equally important was bioinformatics expertise and guidance, for which I would like to thank Stefan, Jerzy and the other members of the Bonn lab.

I would also like to thank Michael and Sandra for making it all possible through the IMPRS Neurosicence program and the members of my thesis committee for critical comments and guidance.

Last but not least, I would like to thank my friends and family for accompanying me on the journey. 



\section{List of Figures}

Figure 1.1 :Timeline showing the currently available biomarkers for Alzheimer's disease with pathological changes in the brain and clinical disease stages.

Figure 1.2 : Scheme demonstrating cellular secretion of exosomes and membrane-derived vesicles. 35

Figure 3.1.1 :Aging is associated with increased variability in water maze task performance ..............57

Figure 3.1.2 No increase in variability on other learning tasks, visual or motor skills...........................57

Figure 3.1.3.1 Individual performance on the water maze task is consistent....................................59

Figure 3.1.3.2 Individual performance on the fear conditioning task is consistent ...............................61

Figure 3.1.3.3 No correlations exist between performance on different memory tasks........................66

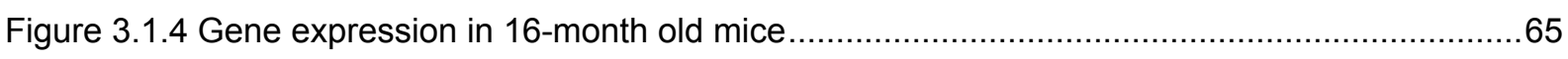

Figure 3.1.5 Gene sets that correlate with memory performance ................................................65

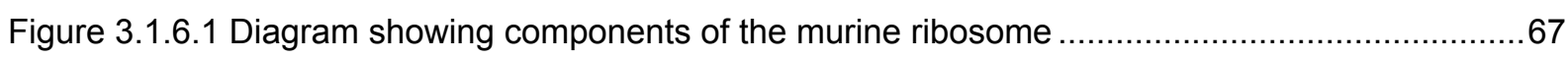

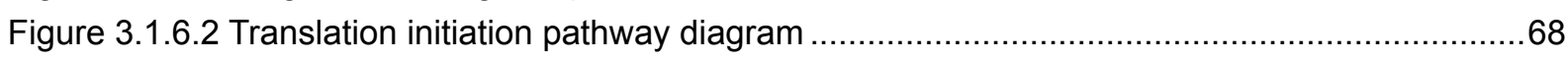

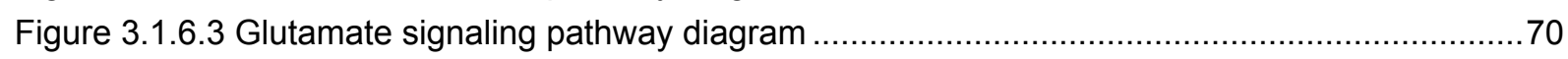

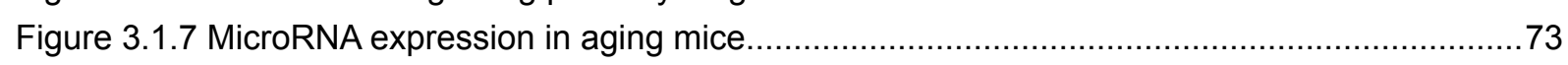

Figure 3.2.1 microRNA expression in the APP-PS1 mouse model .................................................75

Figure 3.2.2 RNA isolation from human cerebrospinal fluid .......................................................

Figure 3.2.3.1 Exosomal RNA bioanalyzer profile .................................................................

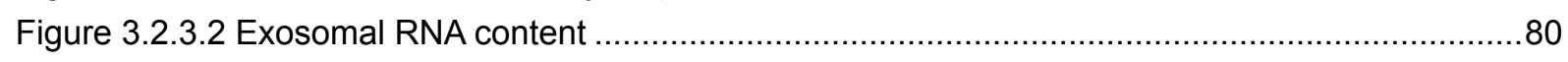

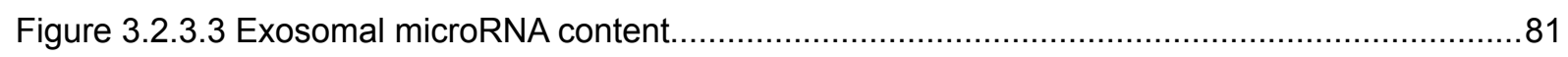

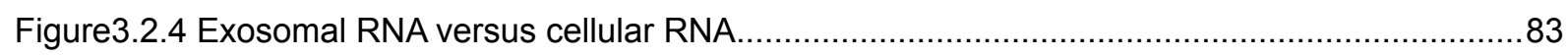

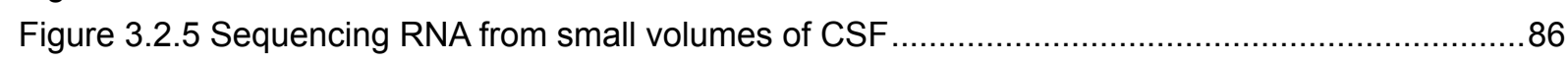

Figure 3.2.6.1 Differential expression and unsupervised clustering of CSF exosomal RNA from AD

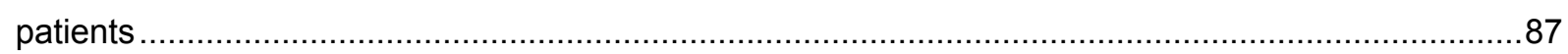

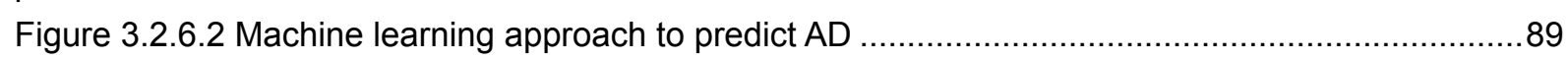

Figure 4.2.1 Comparison between human and mouse studies of $A D$..............................................102 



\section{List of Abbreviations}

\begin{tabular}{|c|c|}
\hline Abbreviation & Long form \\
\hline 3'UTR & 3' untranslated region \\
\hline A-Beta / Aß & Amyloid beta \\
\hline AAMI & Age-associated memory Impairment \\
\hline $\mathrm{ACC}$ & Anterior Cingulate Cortex \\
\hline AD & Alzheimer's disease \\
\hline AMPA & a-Amino-3-hydroxy-5-methyl-4-isoxazolepropionic acid \\
\hline ApoE4 & Apolipoprotien E4 allele \\
\hline APP & Amyloid precursor protein \\
\hline BLAST & basic local alignment search tool \\
\hline $\mathrm{BrDU}$ & 5-bromo-2'-deoxyuridine \\
\hline $\mathrm{CA}$ & Cornu Ammonis \\
\hline CAMK2 alpha & Calmodulin-dependent kinase 2 alpha \\
\hline cDNA & Complementary DNA \\
\hline CNS & Central Nervous System \\
\hline CREB & cAMP response element-binding protein \\
\hline DG & Dentate gyrus \\
\hline DNA & DeoxyRibonucleic Acid \\
\hline DNMT & DNA Methyl Transferase \\
\hline EDTA & Ethylenediaminetetraacetic acid \\
\hline EEG & Electroencephalography \\
\hline EV & Extracellular vesicle \\
\hline fMRI & functional Magnetic Resonance Imaging \\
\hline GluR-1 & AMPA receptor subunit 1 \\
\hline GO & Gene Ontology \\
\hline GSEA & Gene set enrichment analysis \\
\hline HBSS & Hank's buffered saline solution \\
\hline IEG & Intermediate early gene \\
\hline IPA & Ingenuity pathway analysis \\
\hline KEGG & Kyoto Encyclopedia of Genes and Genomes \\
\hline LTD & Long-term depression \\
\hline LTP & Long-term Potentiation \\
\hline MEM & Modified Eagle's medium \\
\hline mGluR & Metabotropic glutamate receptor \\
\hline miRNA & microRNA \\
\hline mRNA & messenger RNA \\
\hline mTOR & mammalian Target of Rapamycin \\
\hline MTT & 3-(4,5-Dimethylthiazol-2-yl)-2,5-Diphenyltetrazolium Bromide \\
\hline NGS & Next-Generation Sequencing \\
\hline NMDA & N-Methyl-D-aspartic acid \\
\hline $\mathrm{nt}$ & nucleotides \\
\hline PCR & polymerase chain reaction \\
\hline PD & Parkinson's disease \\
\hline PFC & Prefrontal Cortex \\
\hline PSD95 & postsynaptic density protein 95 \\
\hline qPCR & quantitiative PCR or real-time PCR \\
\hline RBP & RNA-binding protein \\
\hline REST & RE1 silencing transcription factor \\
\hline RNA & Ribonucleic Acid \\
\hline TAM & Tool for analysis of microRNAs \\
\hline TMS & Trans-cranial magnetic stimulation \\
\hline tPA & tissue plasminogen activator \\
\hline UPL & Universal Probe Library \\
\hline
\end{tabular}



1. Introduction 



\subsection{Learning, memory and age-associated memory impairment (AAMI)}

The complex process of memory acquisition and recall in the brain has been studied for decades, but we are still far from completely understanding it. With aging, the ability to learn and remember tends to decline, in a phenomenon called age-associated memory impairment. Alzheimer's disease, for which age is the biggest risk factor, is characterized by a more severe memory loss and neurodegeneration. Just as in humans, aging rodents too show a decline in the ability to learn and remember, and the hippocampus plays an important role in the neurobiology of these deficits. The following sections deal with the cellular, molecular and behavioral aspects of memory formation and impairment.

\subsubsection{The hippocampus in learning and memory and AAMI}

The hippocampal formation, a part of the 3-layered archicortex and widely held to be important in memory, information consolidation and spatial navigation is present in all mammals. Counterparts of the hippocampus are however found in fish, reptiles and birds where they mediate spatial cognition in ways similar to those in mammals [1]. Thus the mechanisms involved in hippocampus-dependent spatial learning should have evolved relatively early. The neocortical areas, which occupy the largest proportion of the human brain by volume evolved later, and are responsible for higher functions such as thought, language and sensorimotor functions [2].

The prefrontal cortex (PFC), which is greatly expanded in humans and non-human primates controls executive functions, personality, and social behavior [3]. The dorsal PFC has extensive connections with brain areas involved in [4] cognitive and attention-related processes and has been shown to be involved in working memory [5]. Primate studies have shown that the role of the PFC in memory or short term memory is largely that of selective attention rather than memory storage [6]. The prefrontal cortex does not exist in mice, but the anterior cingulate cortex (ACC) has been studied extensively in rodent cognition and plays an important role in learning and memory. In humans, the ACC is involved in error detection [7] and rewardbased decision making [5] but studies linking it with memory are scarce. In contrast, the ACC has been shown to be involved in consolidation and long-term storage of object and location memory $[8,9]$ as well as in contextual fear memory [10] in mice.

The hippocampus has been established as a key player in short-term memory as well as long-term memory. The initial basis for investigating its role in memory was the famous study on the 'Patient H.M.' in whom surgical destruction of the hippocampus led to an inability to form or recall short-term memories while older events were well-preserved [11]. In rodents and non-human primates, a series of ablation experiments demonstrated its role in spatial learning [12], associative learning and recognition [13] and in the consolidation of fear memory [14].

The cellular structure and circuits in the hippocampus make it a particularly favorable area for the electrophysiological study of LTP, and innumerable studies have focused on the rodent hippocampus in an attempt to elucidate the molecular mechanisms involved in neuronal excitation, LTP and synaptic plasticity (reviewed in $[15,16]$ ). The murine hippocampal formation is also anatomically distinct and can be isolated 
for molecular studies with relative ease, facilitating precise and reproducible proteome or RNA profiling. The seahorse-shaped structure, located in the medial temporal lobe of the human brain and an analogous area in the murine brain, shows very similar circuitry in both species. It is composed of the subfields dentate gyrus (DG) and cornu ammonis (CA), which is in turn further divided into the CA1, CA2, CA3. All subfields consist of several strata, organized depthwise. The hippocampal pyramidal neurons are large, and the stratum pyramidale in the CA regions, packed with pyramidal cell bodies is clearly distinguishable by microscopy. Similarly, the stratum granulosum of the dentate gyrus contains the cell bodies of the granule cells. The main input to the hippocampus is from the entorhinal cortex, transmitted via the perforant path to the dentate gyrus and CA3. The axons of the granule cells of the dentate gyrus - termed mossy fibers - terminate on the pyramidal cells of the CA1, which in turn send their axons to the subiculum and entorhinal cortex. Thus, the hippocampus forms an anatomical circuit loop, but doubtless also contains other, smaller pathways of information flow. In various species, hippocampal connections to the amygdala [17], the septum, basal ganglia, hyptothalamus, ventral tegmental area [18] prefrontal cortex [19]and the cerebellum [20] have been demonstrated.

The cortico-hippocampal connections and the perforant path are very well characterized, and play a critical role in memory formation [21]. The Schaffer collaterals, the axons of CA3 pyramidal cells have also been important to the current understanding of synaptic plasticity. The synapse formed by the CA3 pyramidal axons at the CA1 has been used as a model synapse in several studies of LTP and its place in learning and memory has been studied extensively [22]. In mice, the dorsal and ventral regions of the hippocampus have been shown to exert distinct functions. The dorsal hippocampus is shown to be involved in spatial memory [23] while the ventral hippocampus is more closely connected to the amygdala and plays a role in fear and stress responses [24].

Another important reason for studying the molecular physiology and pathology of the hippocampus is its role in Alzheimer's disease (AD). The hippocampal formation and its connections are affected early and extensively in $A D$. The hippocampus is a focal point of the pattern of plaques, neurofibrillary pathology and neuronal loss that characterize AD [25-27]. The gradual loss of memory abilities that constitute the earliest clinical signs of $A D$ highlight the link between memory, $A D$ and the hippocampus.

Interestingly, the dentate gyrus of the hippocampus is also one of the few sites where adult neurogenesis or the formation of new neurons and their incorporation into circuits in the adult brain has been established $[28,29]$. These adult-born neurons are now being increasingly shown to contribute to learning and memory $[30,31]$. The later-evolving cortical areas characterize human behavior, distinguish it from that of other mammals and clearly play a role in learning and the formation and storage of memory. However, the hippocampus still holds an important place in learning and memory. The earliest pathological signs of Alzheimer's disease, whose initial symptoms consist of memory decline occur in the hippocampus [25].

For the above reasons, the behavioral, and especially the molecular analysis described in this thesis is focused largely on the hippocampus. 


\subsubsection{Synaptic plasticity and transcription}

\subsubsection{Synaptic plasticity}

The concept of synaptic plasticity is the foundation of our understanding of the neuronal mechanisms of learning and memory. Synaptic plasticity is the ability of neurons to respond to changes in activity by strengthening certain synaptic connections while weakening others [32]. These changes in the neuronal connectivity are thought to underlie learning in response to environmental stimuli [15].

Synaptic plasticity may be short-term, mediated by an increase in the amount of neurotransmitter available at the presynape, or long-term, which is more commonly studied in the context of learning and memory. The best known mechanisms of long term synaptic plasticity are long-term potentiation (LTP) and long term depression (LTD). LTP is the potentiation or enhancement of postsynaptic response induced by certain presynaptic stimulation patterns [33, 34]. LTD is also activity-dependent, but is characterized by a reduction in the efficiency of synaptic transmission at the involved synapses [35].

An important characteristic of LTP is its persistence, lasting from several minutes to several months [36]. LTP consists of 2 phases, early LTP and late LTP. Early LTP is the facilitation of transmission by activating postsynaptic receptors through phosphorylation, and increased presynaptic neurotransmitter release, mediated through postsynaptic feedback to the presynapse. The critical event in late LTP is new protein synthesis, which enables structural synaptic changes and the formation of new synapses. It has been shown using protein synthesis inhibitors that blocking this step prevents the development of long-term memory [37]. Experiments using RNA synthesis inhibitors have demonstrated that new RNA transcription is also essential for the maintenance of LTP [38, 39]. Late LTP can be further divided into 2 phases - the first can be mediated purely by new protein synthesis in the absence of RNA transcription, and the second requires both [40].

The biochemical signaling cascades in the induction of LTP involve several mediators, among them are the extracellular signal-regulated kinase (ERK) family of mitogen-activated protein kinases.

ERK is persistently activated during LTP and cause a number of downstream events. Another important event is the activation of CAMP response element-binding protein (CREB). The activation of this transcription factor plays a key role in initiating the RNA transcription necessary for LTP and is crucial to memory formation in many different organisms [41].

Initial research on protein synthesis in LTP focused on translation occurring centrally and regulated via universal mechanisms. Mechanisms such as the synaptic tagging and capture hypothesis [42] were developed to describe how overall increases in protein synthesis could lead to the specific activation and strengthening of selected synapses.

Subsequently, decades after the discovery that ribosomal particles are seen in dendrites [43], it became clear that in neurons, activity-dependent protein synthesis occurs locally after transport of the relevant mRNA to the dendrite [44]. This mechanism enables synapses to exert control on protein synthesis, and allows for more rapid availability of new proteins at the synapse [45]. There is also evidence to suggest that 
dendritic protein synthesis is important in memory consolidation; when dendritic targeting of the plasticityrelevant CaMKII-alpha mRNA was blocked, reduction in LTP was observed along with impairments in spatial and associative memory.

\subsubsection{Immediate early genes}

The first wave of cellular transcriptional response to stimuli is by the activation of a set of genes called immediate early genes (IEGs). First described in viruses [46], the IEG response occurs in a variety of cell types such as lymphocytes [47], hepatocytes and cardiac cells [48] as well as in neurons [49]. Most IEGs are transcription factors like $c-F o s, c-J u n$ and $c-M y c$, and Egr1 but the list also includes non-transcriptionfactor genes such a tissue plasminogen activator (tPA) [50] and $\operatorname{Arc}$ [51].

IEGs are known to play an important role in LTP, and are important mediators of learning and memory. The role of IEGs in neuronal plasticity was first revealed after the discovery that LTP-inducing NMDA receptor stimulation also caused the upregulation of the IEG Zif268 [49]. Zif268 was later shown to be essential for late LTP and the maintenence of long-term memory [52]. Increase in expression of other IEGs such as c-Fos during acquisition and early phases of consolidation of memory has been also shown to be a part of learning behavior in a number of vertebrates [53]. A subset of these IEGs are seen to be induced again during reconsolidation of memory [54].

There are also other critical mediators of the neuronal transcriptional response to a learning stimulus. One of these is CREB. CRE-dependent gene expression is pivotal to the learning-induced gene expression program. In mice the CRE- response is seen in the specific brain areas responsible for the type of learning being undertaken. For example, hippocampus-dependent learning such as associative learning or contextual conditioning is accompanied by increases in CRE-dependent gene expression in CA1 and CA3 areas of the hippocampus while the in amygdala-dependent learning paradigms such as auditory-cue fear conditioning the increase is specific to the amygdala [55]. Thus a specific neuronal transcriptional response is an integral part of learning and memory encoding.

\subsubsection{Brain gene expression changes in aging and Alzheimer's disease}

Large-scale transcriptome studies of the brain have enabled us to begin to understand several aging and cognition-related phenotypes. Aging brings about a change in the transcriptional response of the brain. In mice, profiling these patterns in various brain regions revealed that the extent to which aging affects different brain regions varies widely, with prominent effects in both the cortex and the hippocampus [56]. Similar studies in humans show that the in the sixth and seventh decades, there start to be more and more changes in gene expression patterns in various cortical regions [57]. In the human frontal cortex, aging is accompanied by lowered expression of several genes that play a role in synaptic plasticity in the human prefrontal cortex and the increased expression of genes involved in the response to oxidative stress, inflammation and lipid metabolism. These changes start after age 40 [58].

Another study of the human prefrontal cortex provided evidence that those genes which decrease with aging are likely to be of neuronal origin, while those which increase are more likely to be of glial origin [59]. A study 
comparing age-associated transcriptional changes across and 4 human brain regions in different cohorts suggested an overlap between age-related changes and those associated with neurodegenerative disease [60]. Further evidence that the transcriptional program that drives aging is a large component of the one that drives neurodegenerative diseases comes from a microarray analysis of temporal lobe gene expression in $A D$ patients [61], and a systems biology study comparing the gene networks affected in Alzheimer's disease and aging [62]. The former study found a large overlap between the genes differentially expressed in aging and Alzheimer's disease and the latter showed that both processes shared gene expression derangements in mitochondrial processes and synaptic plasticity.

Interestingly, there is evidence that these baseline changes are relatively small in comparison to the transcriptional changes that occur during a response to stimulus. The gene expression changes which accompany aging in the murine hippocampus have been studied by microarray, with the results that at baseline, there are few differences in gene expression pattern between young mice that perform well on behavioral tasks and older mice that are impaired. There is however, a large impairment in the upregulation of gene transcription in old animals in response to a learning-relevant stimulus. Young mice show a robust learning-associated transcriptional response (2229 genes), which is greatly attenuated in aged, learningimpaired animals (6 genes) [63]. Similarly, in the rat DG gene expression changes are seen with aging (around 1000 genes) and the aging-associated changes relate mainly to neurogenesis [64]. This large stimulus-induced transcriptional response appears to be a key event in memory formation, because treatment with histone deacetylase inhibitors that increase the expression of some of these genes was able to reinstate associative memory in the aged mice [63]

\subsubsection{MicroRNAs in learning and memory}

MicroRNAs are a class of small (22-26 nt) regulatory RNAs that act post-transcriptionally by binding to complementary mRNAs. They can directly block mRNA translation to protein or induce mRNA degradation by targeting it to a degradation complex called the RNA-induced silencing complex [65]. Since the relatively recent discovery of this class of non-coding RNAs [66] they have been studied widely and their role in a host of physiological and pathological processes such as embryonic development [67], hereditary disease [68], various kinds of cancer [69], and synapse formation in the nervous system [70] has been established. The latest version of the microRNA database contains more than 2500 human microRNAs, and the process of new microRNA discovery and annotation is still ongoing [71].

After microRNAs started attracting interest as key regulators of translation, there have been several investigations into their role in CNS processes. Most of these studies have involved the selection of a single microRNA or a handful of microRNAs based on a screening procedure. The neuronal function of these microRNAs and their effect on target mRNAs has then been demonstrated by experimental manipulation through microRNA mimics or inhibitors in various model systems.

One of the first genome-scale studies of microRNAs in synaptic plasticity was carried out in the Aplysia synapse [72]. The authors showed that miR-124, a highly conserved brain-enriched microRNA regulates transcription factor CREB, which is responsible for the gene expression changes underlying the induction of long-term potentiation. In mice the expression of CREB was also later shown to be regulated by the brain-specific microRNA miR-134. The deacetylase sirtuin1 was shown to be upstream of miR-134 and to 
regulate the expression of CREB through this microRNA [73]. MiR-34b, another brain-enriched microRNA [72] was also shown to target CREB, albeit in acute myeloid leukemia cells [74]. Thus more than one microRNA has been demonstrated via overexpression or inhibition to act on the same target. However, in the physiological situation it is not always clear if the microRNAs act alone, in concert with one another, or if one or both of them are critical.

CREB also acts upstream of microRNAs. The transcription of miR-132 is upregulated via CREB in an activity-dependent manner in hippocampal neurons in culture [75]. miR-132 has since shown to play a role in dendritic spine formation [76]. Mir-138, another brain-enriched microRNA, was also shown to play a role in dendritic spine formation and maturation [77]. A bioinformatic study of plasticity-related transcription factors and miRNAs has suggested that CREB is one hub of a regulatory network that comprises several microRNAs and the RE1 silencing transcription factor (REST). The analysis reveals that microRNAs and REST form a double negative feedback loop, and CREB acts as a positive regulator [78]. Such a circuit was later shown to regulate miR-9-2 during neuronal differentiation in vitro [79].

Certain microRNAs are known to be concentrated at the synapse and it has been suggested that they play a role in the regulation of local translation [70]. A large number of microRNAs have been shown by immunoprecipitation to be accociated with polyribosome complexes in neurons [80], and many of these are known to be synapse enriched [70]. Moreover, proteins such as FMR1, Dicer and Argonaute which are tightly coupled with microRNA biogenesis and action have been found in granules at the synapse $[81,82]$. MicroRNAs also have role in the neurons beyond the direct regulation of synaptic function and plasticity. The microRNA cluster 17-92 and miR-9 have been shown to be critical for axonal outgrowth and development [83, 84].

\section{Mir 34 family}

One family of microRNAs that is particularly interesting in the context of learning, age-associated memory impairment and $A D$ is the miR-34a/b/c family. MiR-34c in enriched in the murine hippocampus, and a large of overlap is seen when its targets are matched against a list of learning-related genes. Its levels are raised in human AD, as also in aged mice in the APP-PS1 mouse model of amyloid-induced learning impairment. Moreover, inhibiting miR-34c rescues memory impairment in mice [85]. MiR-34a is known to be raised in peripheral blood cells from AD patients [86]. This miRNA has also been reported as a marker of 'brain aging', with levels increasing concomitantly in the brain and in peripheral blood with aging [87]. Interestingly, miR-34a is relatively decreased in the brains of long-lived mice subjected to caloric restriction [88]. The underlying mechanism could be an evolutionarily conserved one, since miR-34 has also been implicated in age-associated neurodegenerative changes in Drosophila [89].

\section{MicroRNAs-mRNAs regulatory network}

Though most studies so far have focused on single microRNAs and their targets, most microRNAs have several predicted targets. Conversely, most genes have more than one microRNA binding site. Mouse knockouts of Dicer, the enzyme critical for miRNA processing, have demonstrated that microRNA species 
as a whole are essential to the survival of cells and organisms [90, 91]. However in many cases knocking out individual microRNAs leads to no or very minor deficits. In C. elegans, where microRNAs were first discovered, a large scale deletion study revealed that the large majority of individual knockouts do not show an overt phenotype under basal condition [92]. In mice, two or more microRNAs must often be knocked out together before a phenotype can be distinguished, showing that they are functionally redundant [93, 94]. Mice with the miR-34 family deleted showed that the knockout has no effect on p53 responses, although p53 has been demonstrated several times in vitro to target miR-34 [95].

MicroRNAs are highly evolutionarily conserved, yet individual microRNAs appear to be functionally dispensable, with a few possible exceptions [96]. Quantititative proteomic approaches have shown that individual microRNAs regulate the expression of hundreds of proteins at at time, but each only to a small degree [97, 98]. For these and other reasons, a new theory of microRNA function suggests that most microRNAs exert a buffering effect rather than acting individually as direct and essential regulatory elements. The more traditional 'tuning' model of microRNA function suggests that microRNAs act to reset the expression of their target genes to a predetermined level through simple repression or through a feedback loop. By contrast the 'buffering' model suggests that microRNAs act to reduce fluctuation in gene expression [96]. The fact that microRNAs preferentially target genes that are have stable expression levels provides additional evidence for the buffering mechanism [99].

For this reason, in order to gain an understanding of underlying cellular mechanisms in disease, a genomewide approach to detect changes in the set of microRNAs seems appropriate.

\subsubsection{Mouse models in the study of learning and memory}

A wide variety of model systems have been used for studying brain function including cultured cells, invertebrates such Drosophila, Aplysia, and C.elegans, as well as higher organisms like mice, rats, and primates. Each of these have a unique set of advantages and disadvantages. Rodents are especially popular models because they are small and easy to breed while maintaining a high degree of functional homology to humans at molecular, cellular and organ-system levels. Particularly relevant to the study of learning and memory, rodents are capable of displaying complex behavior, and various tests of learning and memory have been developed and tested in them. Further, the brain regions and the cellular circuits involved in some memory tasks have been studied in detail in mice and rats. The behavior paradigms we used in this study to assess learning and memory - the water maze task, the fear conditioning task, and the novel object recognition task (described in more detail in materials and methods) - are robust and reproducible tests, whose interpretation has been standardized through many years of observation. The Morris water maze navigation task tests the ability of mice to learn and remember the location of a platform hidden just underneath the surface of water in a pool [100]. Over several days, the escape latency or the time required to find the platform decreases as the mice learn its location. This is a hippocampusdependent task [101]. The fear conditioning task is a test of associative memory, that quantifies memory of an aversive stimulus by recording freezing behavior when mice are placed in the context associated with the stimulus. The fear conditioning task is thought to involve both the amygdala [102] and the hippocampus [103]. The novel object recognition task involves multiple brain regions and tests the ability of mice to recognize a previously seen object and differentiate it from a novel one [104]. While these are the 3 tasks used in this study, there are a large number of other tasks that have been designed to test various aspects 
of rodent learning and memory.

However, mice clearly do not have the same range of cognitive and memory capabilities as humans and some of the higher cognitive and behavioral capabilities such as semantic or declarative memory can not be tested in mice. Mice also lack a prefrontal cortex, that is critical for complex behavioral planning and higher order functions in humans. Thus, in spite of their similarities in brain structure and molecular function, there are many critical differences behavioral and molecular results from mice can not be directly extrapolated to humans.

In spite of their limitations, mice are a particularly useful model for the study of age-associated memory impairment in the context of this study. Like humans, mice show age-associated impairment in memory function $[63,105,106]$. Since aging is a long, continuous process the age-related deleterious effects seen on the brain and other organs are the result of many cumulative events. Both environmental and genetic effects play a role in aging. Since the aim of this study was to study the transcriptional changes associated with aging, we decided to eliminate the confounding factor of genetic variability. Mice were therefore a particularly useful model for this purpose, as a ready supply of inbred animals with virtually identical genotypes are readily available. Another advantage of using mice for this study was that their environment can be controlled to a great degree of precision, and factors such as stress which could contribute to cognitive changes can be carefully monitored. This is particularly relevant for the study of intragroup variability, which forms a key part of the method we used

\subsubsection{Genome-wide epigenetic and gene expression profiling as a method to study disease pathophysiology}

Transcription of the genome into RNA is the first step to building the diverse and complex systems that comprise an organism. However, the vast majority of the human genome codes for sequences to which no function has been ascribed, and protein-coding genes form only a small fraction of the human genome [107]. Ribosomal RNA and transfer RNA are well known to be critical to transcription and translation, and as we begin to understand the function of other non-coding RNA (ncRNA) transcripts it becomes clear that RNAs such as microRNAs, snoRNAs, and long non-coding RNAs also play important regulatory roles. This regulation occurs both in trans-, such as by microRNA binding to complementary regions on mRNA and in cis-, such as by ncRNAs embedded in the 5' UTRs or internal ribosomal entry sites of genes.

RNA is constantly transcribed from DNA and translated into protein. It is also subject to turnover by various intracellular mechanisms and the intracellular RNA profile is a dynamic index of the current state of the cell. Thus profiling the cellular RNA gives a snapshot view of not only the proteins which are poised for production but also of the regulatory or pathological cellular response mechanisms. The available RNA detected by any quantification method is thus controlled not only by the rate of transcription of that particular gene, but also by the stability of the transcript or the rate at which it is degraded. One of the earliest techniques used for the quantitative study of RNA was the Northern blot, where RNA is separated by electrophoretic mobility, transferred onto a membrane and then detected with a complementary fluorescent or electrochemically labeled probe [108]. Though this method is more cumbersome and less sensitive than the newer RNA quantification methods, it is has the advantage of high specificity, and unlike most newer methods, it does 
not involve the reverse transcription and PCR amplification steps before detection [109]. The real-time PCR or qPCR method that is still arguably the most commonly used for RNA quantification involves cDNA synthesis by reverse transcription and then PCR amplification with real-time detection of the amplification. Quantification of qPCR data is usually relative, performed with reference to a housekeeping gene whose levels are assumed to remain constant. Thus ensuring the stability of expression of the reference genes in the specific experimental condition is critical to accurate quantification by this method [110]. qPCR is an extremely sensitive technique, and has been adapted for higher throughput experiments. Standardized or custom-designed qPCR arrays designed to simultaneously amplify hundreds of transcripts are now available and are commonly used in large-scale experiments [111, 112].

In the last 2 decades, there have been major technological advances in the techniques available to study transcription on a large scale. The phrase 'genome-wide' when used in the context of large-scale RNA studies usually refers to the microarray and Next Generation sequencing (NGS) techniques. Microarrays use chips spotted or printed at specific locations with thousands of oligonucleotide probes complementary to known RNA transcripts. After labeled cDNA binds to the probes, the amount of cDNA corresponding to a particular transcript can be quantified by fluorescence intensity at the relevant spot. While the microarray technique was the first to allow genome-scale studies of transcription, it is less sensitive than the amplification-based techniques and the minimum material required for microarrays can be a constraint to certain experimental designs. The advent of next-generation- or massively parallel sequencing has further accelerated the amount of RNA data that can be derived from a single experiment. This method is rapidly taking over from microarrays as the method of choice for genome-wide RNA profiling, by generating larger and larger volumes of data from decreasing amounts of starting material [113]. NGS reads the sequences in a given cDNA library by parallel de novo construction of complementary oligonucleotide sequences, followed usually by amplification and detection by fluorescent reporters. This "sequencing by synthesis" method is the most common but alternative NGS technologies exist that use other methods.

\section{Advantages and challenges in next-generation sequencing}

The greatest advantage of NGS over microarray techniques is that it uses a de novo sequencing approach. This allows the discovery and annotation of novel RNA, the detection of isoforms and partial transcripts, the study of repeat regions of the genome, as well as enables the study of post-transcriptional changes such as RNA editing [113].

NGS technology is currently still developing rapidly, with advances such as increased read length and lower requirements for starting material, enabling its application in a wider range of experimental settings. Moreover the rapidly decreasing costs of NGS experiments will likely enable this technology to be used not only in the research lab, but also in clinical diagnostics [114]. Two major advantages of genomewide approaches to transcription stand out. First, they allow an unbiased and exhaustive screening of transcriptional and epigenetic events associated with a biological phenomenon. This absence of an a priori selection bias for well-known or previously studied genes, has allowed researchers have been able to challenge existing dogma about important pathways associated with biological phenomena and led to the discovery of new types of non-coding RNA such as competing endogenous RNA and circRNA [115, 116]. Second, the observation of a large numbers of concurrent transcriptional events allows us to better understand the interrelationships between genes. This provides a vast resource for the construction of intracellular regulatory network models. 
A special advantage of next-generation sequencing that is particularly relevant when studying RNA in special cellular compartments such as exosomes is that it allows for direct quantification of the number of RNA transcripts. Sequencing techniques are not completely free from bias caused by selective amplification. However, in a situation where there are no 'housekeeping genes' that can be used to normalize expression levels, sequencing provides a more reliable estimate than QPCR or arrays that are dependent on the affinity of individual probes to the RNA transcript.

When using these NGS to study transcription, the methods used for data analysis need careful consideration and can be critical to the results of the experiment. Raw data generated by the sequencer needs to be demultiplexed, checked for quality, and aligned to the genome of interest before a meaningful indication of RNA expression can be obtained. Many of these tasks are computationally intensive and require expertise. Algorithms for the mapping and counting of non-coding RNA have still not been standardized, and the development of new bioinformatic methods to tackle these challenges is ongoing.

However the challenges posed by the handling large amounts of data generated by high throughput sequencing technology are offset by its many advantages. 


\subsection{Molecular Mechanisms of Alzheimer's disease (AD)}

\subsubsection{Alzheimer's disease: Causes and hypotheses}

Over the years, there have been a few different hypotheses to explain the etiopathogenesis of Alzheimer's disease $(A D)$, with lines of evidence to support each one.

The acetylcholine hypothesis, originating in the 1970s, suggests that there is a deficiency in cholinergic transmission in AD. This is based on post-mortem biochemical evidence from the brains of AD patients showing a deficiency in neocortical choline-acetyl transferase and presynaptic cholinergic deficit as well as a reduction in excitatory amino acid neurotransmission [117]. The majority of drugs currently used for treatment of $\mathrm{AD}$ (tacrine, rivastigmine, galantamine and donepezil) are are cholinesterase inhibitors, which attempt to increase the available of acetylcholine at the synapse. However their therapeutic efficacy is low $[118,119]$.

The only other drug that is approved for AD treatment is memantine, which targets N-Methyl-D-aspartic acid (NMDA) receptors. This mode of action is based on excitotoxicity, where increased glutamatergic transmission leads to deregulated calcium influxes and apoptosis. Excitotoxicity as a pathological mechanism is common to several neurodegenerative diseases, as well as other CNS diseases such as stroke or traumatic brain or spinal cord injury.

In the decades following its origin the cholinergic hypothesis was challenged, and the amyloid-beta (A $\beta$ ) and tau hypotheses took center stage. These are based on the accumulation of protein aggregates in the brains of patients with AD. The amyloid hypothesis is supported by the fact that familial AD is associated with mutations in the gene coding for amyloid precursor protein (APP) [120] and in the gene coding for a component of the gamma secretase (presenilin1 or PS1) [121] that cleaves APP. Human evidence for the involvement of the microtubule-associated protein tau comes largely from the aggregation of tau tangles in $A D$ brains [122]. However mutations in tau are associated with frontotemporal dementia rather than $A D$ [123]. In mice, there is a large body of evidence suggesting that reduction or knockout of the tau protein ameliorates $A \beta$-induced toxic decline [124].

An interesting recent hypothesis to that is beginning to emerge is that misfolded proteins can be transferred from cell-to-cell in a prion-like manner and spread disease pathology. The evidence for this is largely derived from mice and is as follows. First, the intracerebral innoculation of small amounts of AD brain extract can induce amyloidosis in human APP (hAPP) transgenic mice [125]. This effect is also seen when A $\beta$ extracts are injected intraperitoneally, but not through other routes of administration [126], unlike in prion diseases. The tau protein may also be secreted from cells and spread across synapses [127-129]. On the cellular level, spreading of protein aggregates in manner that is similar but not identical to prions has been well documented $[130,131]$ and the spread of these protein aggregates ( $\alpha$-synuclein) in PD has been researched more than in $A D$. $A \beta$ generated intracellularly has been shown to be released into the extracellular mileu with exosomes [132]. One major difference appears to be that in contrast to prions, there is no evidence that the protein aggregates associated with Parkinson's disease (PD) or AD are infectious or spread from 
person to person.

In addition to these causal hypotheses, a multitude of other factors such as oxidative stress [133], metals and biometals $[134,135]$ is known to contribute to or increase the risk of developing Alzheimer' disease.

\section{$A D$ - One disease or many?}

For each of these proposed mechanisms, there exist cohorts of AD patients who do not fit the pattern, and show no evidence of the associated molecular pathology. At the time that the cholinergic hypothesis was being researched, it emerged that a subset of AD patients did not show significant cholinergic deficits [136]. Similarly, though ApoE4 is well known as a risk factor for $A D$, there exist geographically and ethnically distinct subpopulations of $A D$ patients for whom ApoE4 has no association with $A D[137,138]$.

Moreover, Alzheimer's disease is known to progress a differing rates after onset, and a variety of clinical and imaging indicators have been used to predict rates of decline [139]. However the reasons why some patients have slowly progressing disease and others deteriorate rapidly is unknown. Thus these mechanisms may coexist and more than one of them may cause the pathology that leads to disease manifestations such as loss of memory and other cognitive deficits.

Alternatively, rather than being a single disease characterized by all these features, what we know today as Alzheimer's disease may well be a mixture of several diseases. This has been shown to be the case in carcinoma of the breast, which was initially thought of as a single disease, and classified into a handful of types based on tumor morphology and immunohistochemistry. With advances in genomic and gene expression studies it became clear that these each of these cancer types consist of several molecularly defined subtypes and that survival rates and response to therapy often depend critically on genomic classification [140]. These findings led to a new classification system, which is rapidly being adopted in clinical diagnosis and therapeutic decision-making.

This will perhaps one day be the case for other non-malignant diseases too and is one reason why molecular research into the gene expression patterns of $A D$ may provide a clue to its pathogenesis or drive research into its classification. Since tissue for diagnosis is only available post-mortem, finding a way to study molecular mechanisms in humans, particularly the early mechanisms before neuronal loss could prove critical to stratifying disease and identifying effective treatment.

\subsubsection{Age as a risk factor for AD — Parallels and contrasts between AAMI and AD}

Aging is the single largest risk factor for Alzheimer's disease, and it is well known that the prevalence of sporadic AD increases with age [132, 144]. Aging by itself, in the absence of clinically diagnosed $A D$ is accompanied by varying rates of cognitive decline $[145,146]$. Some of the individuals with ageassociated memory impairment (AAMI) will go on to develop dementia, while others will continue to show mild impairment [147]. Since aging and $A D$ are so closely related, it is not clear which pathomechanisms underlie age-associated cognitive deficits in the absence of dementia and how they are different from those that predispose aged individuals to Alzheimer's disease. 
There are several lines of evidence indicating that the brain changes that accompany $A D$ are different from those associated with AAMI. It has been shown by neuropathological methods that $A D$ but not $A A M I$ is associated with neuronal loss in the CA1 region [148]. In contrast, based on imaging studies in a several species, AAMI is thought to originate in the dentate gyrus of the hippocampus, with sparing of the CA1[148, 149]. On the molecular level, levels of soluble versus insoluble Abeta1-40 and Abeta1-42 are thought to differentiate AD from AAMI [150]. The ApoE4 allele that is an important genetic risk factor for AD. However its correlation with AAMI is unclear, with some studies supporting a correlation $[151,152]$ and others suggesting the opposite [153, 154].

Based on rodent and primate studies it has been suggested that AAMI is accompanied by synaptic alterations and failure in the same synaptic circuits that are the sites of marked neurodegeneration and neuronal loss in $A D[155,156]$. Functional magnetic resonance imaging (fMRI) studies show that AAMI is associated with a failure to suppress activity in task-irrelevant brain regions $[157,158]$. This is supported by electroencephalography (EEG), and trans-cranial magnetic stimulation (TMS) data, suggesting that agerelated cognitive impairments stem from an age-associated loss in the ability to suppress the processing of irrelevant stimuli or information [159]. The most recent evidence that AAMI is mechanistically distinct from $A D$ comes from a microarray screen of gene expression profiles in post-mortem human brain tissue, which showed that changes in the expression of the histone binding protein RbAp48 could explain the phenomenon of accelerated memory loss [160].

It is clear that $A D$ is different from $A A M I$, but it is equally clear that $A D$ is rarely seen in the young in the absence of inherited mutations. However, little is known about the mechanisms that cause aging to be a risk factor for $A D$. One possibility is the cumulative effects of agents such as oxidative stress, which has been linked with both AAMI and AD [161].

\subsubsection{Need for new methods to study Alzheimer's disease}

Alzheimer's disease is diagnosed largely on the basis of clinical symptoms and a tissue -based diagnosis is clearly impossible. This has led to large efforts in the development of brain-imaging based technologies for $A D$ diagnosis. However, for a deeper mechanistic understanding of the pathogenesis of $A D$, the development of new ways to study the live human brain is critical.

While there is clear evidence in humans from association studies for some of these mechanisms, such as the involvement of the amyloid precursor protein (APP), the association of the apolipoprotein 4 allele (ApoE4) [141], the bulk of the mechanistic studies have been performed in mice. One clear limitation of using transgenic mice as a model for the disease is that Alzheimer's disease is a human disease, and there is no evidence that the specific pathogenic processes and response mechanisms exist in mice or other species. Another disadvantage is that behavioral tests in mice are relatively crude, there are few methods to detect subtle cognitive changes. Since the cognitive abilities that exist in mice (both for testing and that can potentially be impaired in a disease model) fall far short of humans, there is little reason to believe that mice can successfully be used as a model for the cognitive deficits in AD. The pathways and mechanisms involved in human $A D$, particularly the early cognitive deficits involving executive functions such as attentiveness, planning and abstract thinking [142], as well as language deficits, semantic losses and loss of vocabulary, confusion can scarcely be tested in mice. The types of memory tests that are 
usually performed in mouse models are tests for associative and long term spatial memory, which are only a small part of the constellation of symptoms that accompanies advanced AD. This is perhaps one reason why drugs that are effective in halting disease progression and improving cognitive deficits in mice do not translate well to human disease. In mouse models of $A D$, which are largely based on the amyloid hypothesis, tau accumulation is not a consistent downstream component of the pathology [143]. This is a major difference from amyloid pathology in humans, suggesting that the toxicity of overexpressed $A \beta$ in mice is mediated through different molecular mechanisms than in humans, and is therefore not an appropriate model to study human AD.

\subsubsection{Current state of biomarkers in AD}

Since the search for effective therapies for AD has yet to deliver drugs that succeed in reversing symptoms of modifying disease pathology, it is possible that earlier diagnosis of the disease and insight into the early mechanisms of disease pathophysiology may aid in the search for more effective treatment. The symptoms of $A D$ such as memory decline and confusion appear after losses in neuronal function and integrity have already occurred $[162,163]$. Thus, by the time treatment is initiated, it is often too late to target any early, potentially reversible neuronal changes. Further, since most of our current knowledge of the disease etiopathology is based on post-mortem changes in the brain, our knowledge of the cellular and molecular events in early $A D$ is relatively limited.

Since A-beta plaques and tau tangles are the traditional hallmarks of $A D$, they were also the earliest and best known biomarkers. Cerebrospinal fluid (CSF) levels of amyloid-beta 42 have been shown several times to be significantly lower in patients with AD [164-168]. Levels of tau protein in CSF are known to be raised in patients with $A D[169,170]$. These 2 protein biomarkers in combination have been proven to have sensitivity rates of up to $95 \%$ in the diagnosis of $A D$ [165]. However, their specificity is lower, with $36 \%$ of normal elderly subjects also testing positive [165]. Since the first clinical studies on these biomarkers, longer-duration multicenter studies have proven that there are several factors such as the type of detection kit used and CSF storage conditions which can confound the analysis of these biomarker levels [171173]. Nevertheless, the use of CSF A-beta42 and tau levels is now recommended as a part of the clinical diagnostic workup in $A D$ where possible, although they are neither sufficient nor necessary for establishing the diagnosis [174].

Certain neuroimaging markers are also able to differentiate between patients with $A D$ and healthy control subjects. Magnetic resonance imaging (MRI) measurements of hippocampal volume have been used to predict progression from mild cognitive impairment (MCl) to $A D$ [175] and with varying degrees of efficacy to differentiate $A D$ from fronto-temporal lobar degeneration [176]. Fluorodeoxyglucose Positron Emission Tomography, which images glucose metabolism in various brain regions is able to localize areas of amyloid deposition in the cortex of patients with AD [177]. Technetium-99m single-photon emission computed tomography was able to differentiate patients with $A D$ from those other kinds of dementia with an accuracy of about $75 \%$ [178]. These imaging procedures are also included in the latest revision of the recommended clinical procedures for AD diagnosis [174]. Other novel techniques for early detection of Alzheimer's include non-invasive opthalmoscopic examination of the retina for amyloid plaques [179], and tests for olfactory dysfunction [180]. A recent study showed that 'metabolomics' or a liquid chromatography/mass spectrometry-based profiling of CSF or plasma for levels of metabolites such as amino acids, lipids, and 
peptides could be used to detect a perturbation of metabolic pathways. This method was able to identify patients with $A D$ or mild cognitive impairment from control subjects with a fairly high degree of accuracy [181].

The advantage of molecular markers over neuroimaging is that the former lend themselves better to longerduration multicenter studies, since tests can be performed at a later time after collection and preservation of the CSF or plasma sample. Further, the availability and costs of neuroimaging equipment coud restrict its widespread use in diagnosis. Another advantage of molecular studies is that the candidate biomarkers can be used to further investigate underlying disease-associated mechanisms and to develop targeted therapies. Though there have been several candidates investigated for use as biomarkers for $A D$, the only ones which have been efffectively translated to clinical practice are A-beta and tau levels in CSF. Since these are based on traditional hypotheses of diease mechanism, they are unable to provide any new information about etipathogenesis of the disease. Thus there is plenty of room for the development of newer biomarkers that potentially provide insight into disease mechanisms.
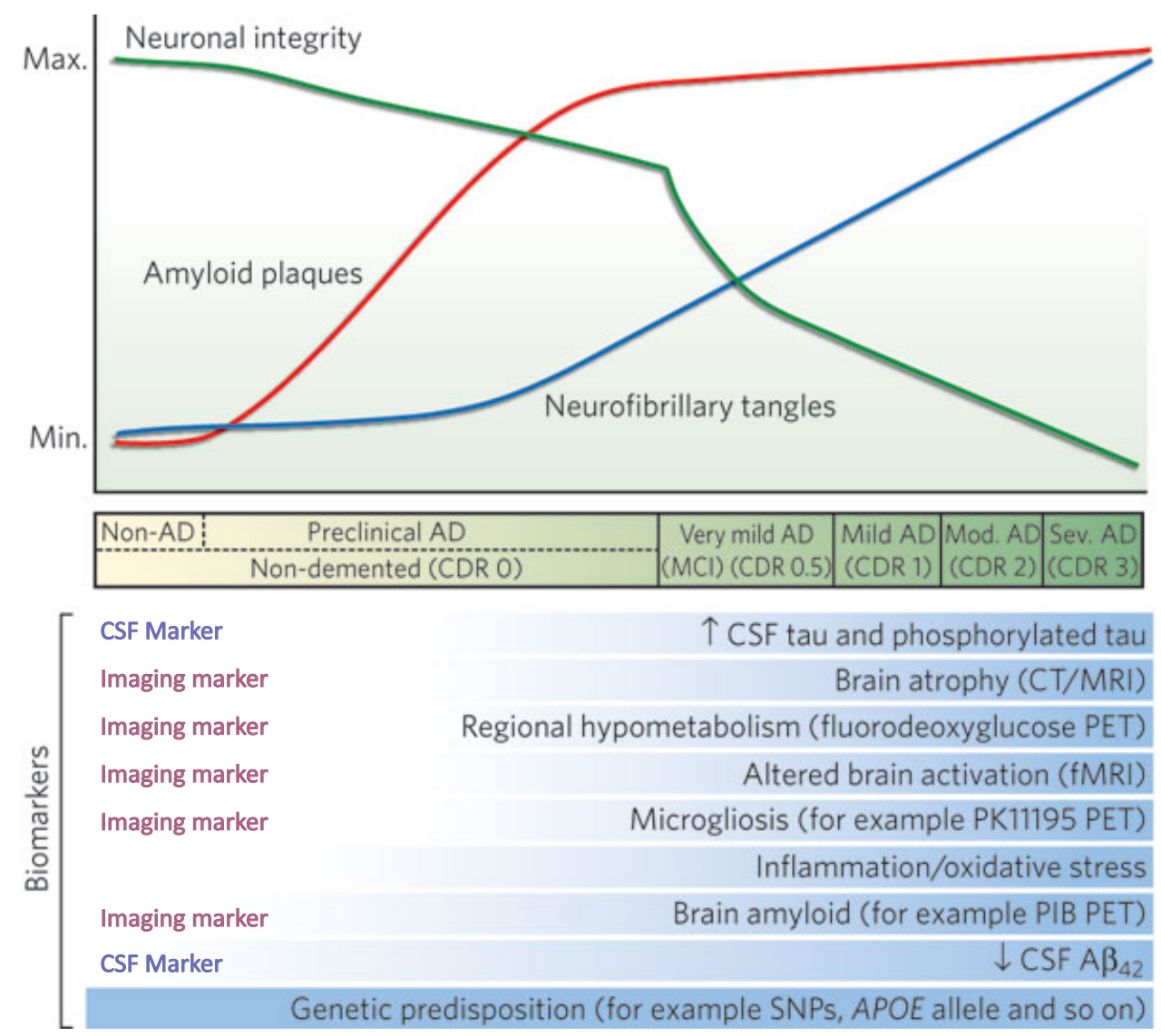

Figure 1.1 :Timeline showing the currently available biomarkers for Alzheimer's disease with pathological changes in the brain and clinical disease stages (Adapted from Perrin et al., Multimodal techniques for diagnosis and prognosis of Alzheimer's disease, Nature 461(916-922) [177]) 


\subsubsection{Extracellular vesicles}

Most cells constitutively secrete exosomes and other types of vesicles into extracellular space. These vesicles may be derived from the intracellular endosomal system, in which case they are called 'exosomes' or may directly bud off from the plasma membrane. These extracellular vesicles (EVs) contain proteins, RNA and cell surface receptors derived from their cell of origin. After the discovery in 2007 that cellderived secreted vesicles are able to transfer mRNA and microRNA between cells and that the mRNA transferred in this manner can function in recipient cells [182], there have been studies showing this to be the case in many different cell types such as hepatoctes [183], immune dendritic cells [184] glioblastoma cells [185], melanoma cells [186], monocytes [187], and embryonic stem cells [188]. In the 5 years between this discovery and the writing of this thesis, there have been a profusion of research and review articles describing the protein and RNA contents of cell-derived vesicles [189, 190].

The fact that cells secrete RNA and protein-containing vesicles that can be isolated from CSF is an untapped opportunity to study brain cellular processes. It is not yet clear if EV content is cargo intended for transport or other cells or unwanted byproducts of cellular processes, targeted for excretion from the cell, or both. In either case, EVs are rich in protein and RNA and studying these is likely to provide an insight into the functioning of the cell of origin. In either case, there is increasing evidence that cells specifically target certain RNAs and proteins to EVs.

\subsubsection{Exosomes and extracellular vesicles in CNS physiology and Pathology}

In the nervous system, evidence has rapidly accumulated in the last few years that exosomes and other extracellular vesicles play a role in physiology, in the healthy state as well as in disease. The importance of their role, and the extent to vesicle-mediated intercellular communication is critical to normal neuronal function is still being researched.

\subsubsection{Physiology}

Secretion of exosomes and other vesicles into the extracellular space is a part of normal function in CNS cells. Neuronal and astroglial cells in primary culture constitutively secrete exosomal vesicles into extracellular medium and exosomal release can be regulated artificially by inducing cellular depolarization by potassium chloride treatment [191] or glutamatergic stimulation [192].

The role of exosomes in intercellular communication in the nervous system has been best studied in the case of neuron-glia interactions. This interaction occurs in both directions, with glial exosomes being taken up by neurons and vice versa. Oligodendrocytes in culture secrete exosomes, which in addition to myelin protein components, contain several proteins that could be potentially neuroprotective [192]. The secretion of these oligodendroglial exosomes can be regulated by medium from neuronal cell cultures [193]. Further, a 2-way communication between neurons and oligodendrocytes has been demonstrated in cultured cells. Oligodendroglial exosomes are released upon glutamatergic neuronal stimulation, and neurons take up the released exosomes, whose contents could have a neuroprotective role [194]. Microglial cells also secrete 


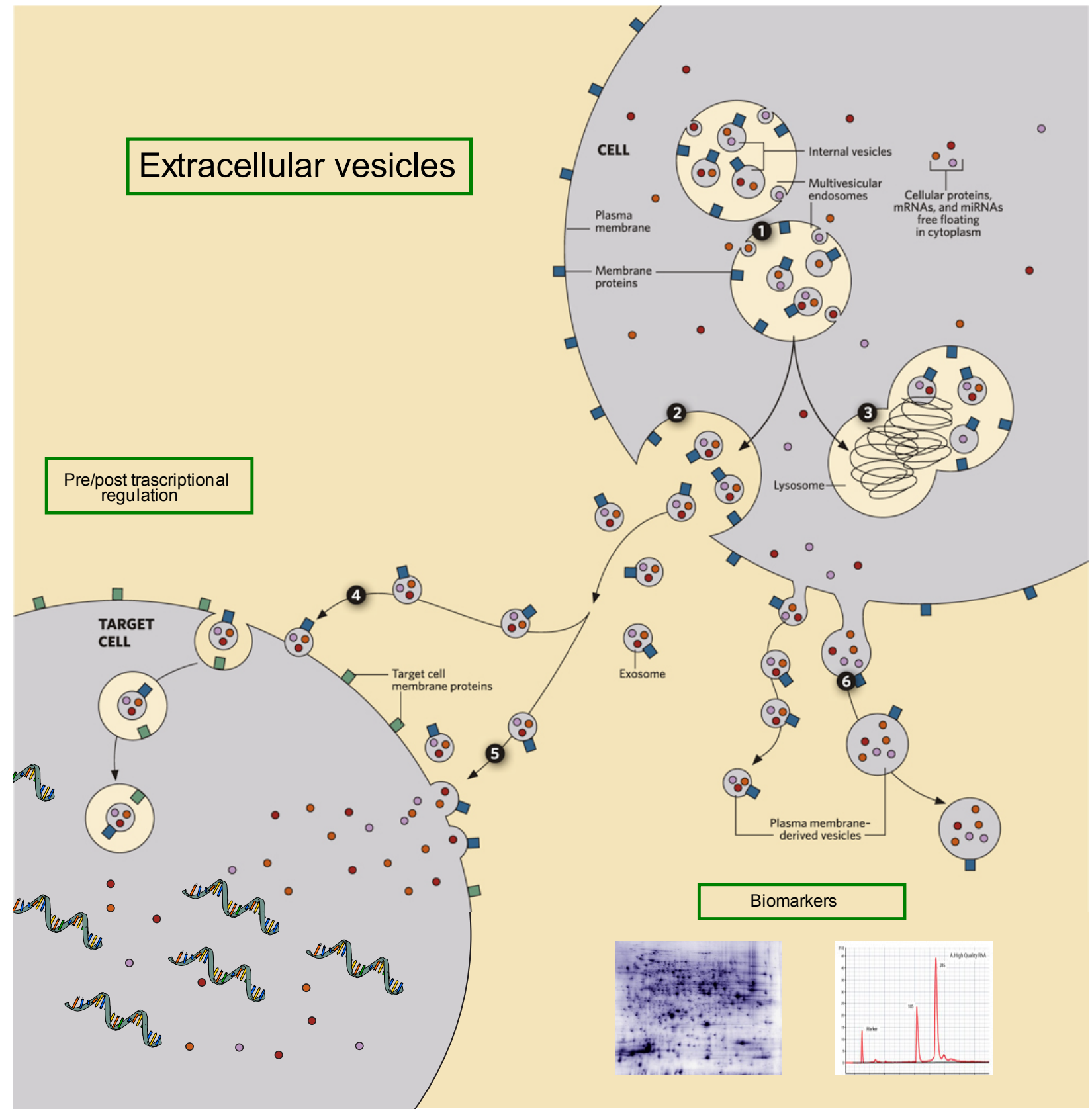

Figure 1.2 : Scheme demonstrating cellular secretion of exosomes and membrane-derived vesicles These vesicles contain protein, RNA and cell-surface receptors, and can interact with the surface of recipient cells or be absorbed into them. The miRNA in these vesicles can act on mRNA in the recipient cells and regulate its translation. Adapted from F1000 Reports, Jul 2011, Exosomes: Secreted vesicles and intercellular communications Clotilde Thery, Institut Curie [206] 
exosomes [194]. Microglia-derived vesicles can modulate synaptic transmission by increasing neuronal excitability and enhancing sphingolipid metabolism [195].

Neuronal exosomes in which microRNA has been overexpressed are taken up by astrocytes and transfer the microRNA to the recipient cells, where it appears to actively elicit a response [196]. In addition to microRNA, it appears that the neuron-astrocyte exchange may also involve protein transfer. There are indications that astrocytes in culture release heat shock protein-containing exosomes, with the corresponding implications for the neurons and other cells in their microenvironment [197, 198]. The role of exosomes in interneuronal communication is yet to be studied in depth, but hints that they play a role in synapse physiology are beginning to emerge. At the drosophila neuromuscular synapse, exosomes secreted at the presynapse are distinct from synaptic vesicles, and mediate trans-synaptic transfer of signaling proteins that are instrumental to synaptic development and plasticity [199].

The cell of origin of an exosome is usually suggested by receptors, surface protein or intravesicular proteins that it carries, such as Glu2/3 receptors suggesting a neuronal or glial source [191] or wnt-3 protein suggesting an embryonic stem cell source [200]. Traditional exosome isolation methods however yield a mixture of different exosome types. Immunostaining for different 'cell markers' allows one to guess at the source of exosomes but does not provide an estimation of the relative proportion of cell types represented. Therefore, exosomes isolated from CSF or plasma are likely to originate from more than one cell type.

Exosomal surface proteins have been used to target the delivery of exosomal contents to specific cells. In a landmark study, Alvarez-Erviti et al. engineered dendritic cell exosomes to express a neuron-specific peptide on their surface and were able to deliver a peripherally injected siRNA to the brain by packaging it in these exosomes [201]. Folate receptor- $\alpha$, expressed on exosomes has also been used to deliver folate into brain parenchyma via CSF [202].

\subsubsection{Pathology}

There are several ways in which extracellular vesicles could be important in the pathogenesis or detection of CNS disease. Scattered evidence showing the role that EVs play in various neurodegenerative diseases is beginning to emerge. With relevance to AD, in APP-transfected N2A cells in culture amyloid beta peptides are released in part, in association with exosomes. Exosomal proteins such as flotillin and Alix colocalize with A-beta plaque sites in the brain [203]. Another connection between exosomes and AD is that AT270, a phosphorylated form of tau that is found in the CSF of $A D$ patients with early $A D$ is secreted from neuronal cells in association with exosomes [204].

Glioma cells containing an oncogenic variant of the epidermal growth factor receptor are able to transfer it via membrane-derived vesicles to cells which originally lacked this receptor. This results in a transformation of signaling pathways and a change in phenotype in the recipient cells [205]. In patients with glioblastoma, extracellular vesicles in the serum were found to contain mRNA corresponding to a tumor-specific splice variant [185]. This suggests that at least in malignancies, vesicles derived from pathological brain tissue are able to reach the blood stream and may be used for diagnosis. The existence of brain-derived vesicles in the blood stream in healthy individuals or in other CNS diseases like AD has not been proven. 
For Parkinson's disease, studies in an alpha-synuclein-secreting neuronal cell line showed that exosomal alpha-synuclein can cause disease pathology in healthy neuronal cells. Further, transmission of alphasynuclein pathology to healthy neurons transplanted into the brains of PD patients [206, 207], gave rise to the hypothesis that extracellular vesicles may be instrumental in the transport of $\alpha$-synuclein aggregates from between connected neurons [208, 209]. Mouse motor neuron cells with mutated superoxide dismutase 1 (the mutation responsible for amyotrophic lateral sclerosis) also secrete it via exosomes. It has also been shown that prion protein is released in association with exosomes and this could be a mechanism by which prions are transferred from cell to cell [210].

Since studies so far suggest that EVs aid intercellular communication and potentially cellular excretion in the CNS, there is vast scope for further investigation into these vesicles and how their contents could serve as an indicator for processes occurring in brain cells. There is still much unknown about the contents of EVs, their relationship to host cells and the potential for their use in gaining a better understanding of CNS function in health and disease. 
2. Objectives 



\subsubsection{RNA in age-related memory impairment}

Aging is associated with a deterioration of various physiological processes, and the loss of memory with aging is a well-known phenomenon. However, the deterioration in memory function is not uniform or predictable, and differs greatly from person to person, with some individuals showing rapid deterioration while others continue to perform well on cognitive tests at older ages. Most animal studies of age-associated memory impairment have studied the aged as a group rather than at the level of the individual. When using this approach, the mechanisms responsible for memory decline are masked by general aging-related changes. In order to investigate the heterogeneity in age-related memory loss and pinpoint some of the signs of healthy cognitive aging, the objectives of this study are:

1. Develop a mouse model that distinguishes benign age-related changes from those associated with cognitive decline

2. Test the model for reproducibility across age groups

3. Study the mRNA and microRNA expression profiles that characterize 'good' and 'poor' learners among aging mice

4. Determine which intracellular pathways are altered in age-related memory impairment

\subsubsection{RNA in Alzheimer's disease}

Alzheimer's disease is one of the most critical causes of disability in old age, and despite its high prevalence, no effective treatment exists. Like other diseases of the central nervous system, in Alzheimer's disease the currently available tools for investigation are limited to post-mortem study of diseased tissue and transgenic animal models. The recent discovery that cells secrete protein- and RNA-containing vesicles into extracellular space opens up new possibilities for studying disease mechanisms in inaccessible tissues such as the brain. The aim of this project was to to investigate the possibility that extracellular RNA in cerebrospinal fluid can be used to study Alzheimer's disease. The objectives of this study are

1. Determine if RNA exists in a stable form in human cerebrospinal fluid and can be reproducibly isolated from it.

2. To optimize the yield of RNA from CSF extracellular vesicles, and study the profile of cerebrospinal fluid RNA by next-generation sequencing

3. Compare cellular and extracellular RNA, determine if extracellular RNA serves as a readout for intracellular events

4. Investigate the potential for use of extracellular RNA as a biomarker for Alzheimer's disease 

2. Methods and Materials 



\subsection{Animals and behavior experiments}

\subsubsection{Animal care}

All mice except the APPPS1 transgenic animals were specific-pathogen-free C57BI6/J wild type mice (Mus musculus, common house mouse) purchased from Janvier SAS. The APPPS1 mice also had a C57BL6/J background and were obtained from a colony maintained in-house. All animals were housed in the animal facility of the European Neuroscience Institute, Göttingen in individually ventilated cages (IVC, $32 \times 16 \times$ $14 \mathrm{~cm}$, Techniplast) with temperature and humidity regulated. They were fed and watered ad libitum with a standard laboratory mouse diet and cages were changed every seven days. The groups of mice used to test the inter-individual variability on the behavioral and molecular level (Project $A$ ) were all housed individually from the point of delivery onwards. All animal care and testing protocols were approved by the Veterinary Institute of the Lower Saxony State Office for Consumer Protection and Food Safety.

\subsubsection{Water maze Task}

A white square platform $(11 \times 11 \mathrm{~cm})$ was placed in a circular pool $1.2 \mathrm{~m}$ in diameter, filled with opacified water at $20^{\circ} \mathrm{C}$ to a level $18 \mathrm{~mm}$ above that of the platform. Four different visual cues were attached around the tank and the mouse was gently placed in the water to look for the platform. Each mouse was allowed 4 trials to find the platform, with a maximum of 60 seconds per trial. If a mouse found the platform within 60 seconds it was allowed to stay there for 10 seconds before starting the next trial. If a mouse did not find the platform in 60 seconds it was placed on the platform and allowed to remain there for 10 seconds before starting the next trial. This procedure was repeated on 5-10 consecutive days to allow the mice to learn the location of the platform. On the final day the platform was removed to perform a probe test for sixty seconds under the same conditions. The path taken by the mice was recorded by a video camera and analyzed by Videomot 2 software (TSE). For purposes of analysis the pool was divided into four quadrants, and probe test readout was the time spent in the quadrant where the platform was. The readout of learning from the training days was average escape latency or time required to find the platform across 4 trials [100].

\subsubsection{Fear Conditioning Task}

Each mouse was placed in a $23 \times 23 \mathrm{~cm}$ metal cage (context) for 3 minutes before being subjected to an electric shock of $0.7 \mathrm{~mA}$ for 2 seconds administered to the feet via the metal bars at the bottom of the cage (TSE multiconditioning system). Associative learning was tested 24 hours later by exposing the mice to the same context for 3 minutes. The relative duration of freezing measured by the software (TSE FCS) using a motion threshold of 18 au and freezing time threshold of $1 \mathrm{~s}$ was used as a readout for memory.

\subsubsection{Novel object recognition test}

The test involved three phases, an initial 2-day habituation phase, a subsequent 2-day similar-object exploration phase and a final day when a novel object was presented. The habituation phase involved 
placing each mouse in an open chamber for 5 minutes each day. During the similar-object exploration phase, two identical blocks of size $3 \times 3 \times 3 \mathrm{~cm}$ were put in the chamber for the mouse to explore. On the fifth day one of the objects was replaced by an unfamiliar object. Based on the propensity of the mice to explore the novel object more than the familiar one, The recognition of novelty, and the resulting tendency to explore the unfamiliar object more than the familiar one is based on the memory of the familiar objects seen on the previous days. The movements of the mouse were tracked by a video camera (Videomot software) and the percentage of time spent exploring the new object was recorded for use as a readout [211].

\subsubsection{Rotarod test}

Motor impairment was assessed by placing the mice on a horizontal rod, rotating around its longitudinal axis (Rota Rod V4.02 System (TSE Systems)). The animals were trained for 2 days at a speed that would require them to cover $10 \mathrm{~cm} / \mathrm{s}$ for 3 minutes 2 times each day. If the mouse fell off the rod during training, it was placed back on it repeatedly until 3 minutes had elapsed. The animals were then tested 4 times over 2 days for 3 minutes, at speeds increasing from $5 \mathrm{~cm} / \mathrm{second}$ to $180 \mathrm{~cm} / \mathrm{sec}$. The time elapsed before the mouse fell off the rod was automatically recorded and the average value obtained from 4 testing sessions was used an indicator of motor skills.

\subsubsection{APPPS1-21 mice}

The APPPS1-21 double transgenic strain generated in the laboratory of Mathias Jucker at the HertieInstitute for Clinical Brain Research, Tübingen, Germany [212] was used as a mouse model of AD. The mice overexpress the gene for amyloid precursor protein (APP) with the 'Swedish' mutation (Lysine to Asparagine at position 670 and Methionine to Leucine at position 671) and the presenilin gene with a Leucine to Proline mutation at position 166 of the protein on a C57BL7/J background. The mutations, which are coexpressed under a neuron-specific Thy-1 promoter lead to early onset of neuropathology with cerebral amyloidosis detectable at the age of 6-8 weeks [227].

\subsubsection{Genotyping}

DNA was isolated from mouse tail biopsies taken at about 3 weeks of age. The tail biopsies were lysed in $200 \mu \mathrm{l}$ of Direct PCR DNA Extraction Reagent (Viagen) with $2 \mu \mathrm{l}$ proteinase $\mathrm{K}(200 \mathrm{mg} / \mathrm{ml})$ at $55^{\circ} \mathrm{C}$ for 6 hours, followed by inactivation at $85^{\circ} \mathrm{C}$ for 45 minutes. The DNA was used for PCR under the following conditions: Denaturation for 5 minutes at $95^{\circ} \mathrm{C}$ followed by 30 cycles of $95^{\circ} \mathrm{C}$ for 30 seconds, $58^{\circ} \mathrm{C}$ for 1 minute, $72^{\circ} \mathrm{C}$ for 1 minute and a final elongation step at $72^{\circ} \mathrm{C}$ for 5 minutes. The reaction mix contained 2.5 $\mu \mathrm{l}$ of 10X Dreamtaq Green buffer (Fermentas), $1 \mu \mathrm{l}$ of 10mM dNTP mix (Thermo Scientific), $1 \mu \mathrm{l}$ of each of the 4 primers at 100 um concentration, $0.05 \mu$ of DreamTaq enzyme (Fermentas), 1-2 $\mu$ l of genomic DNA and water to a total volume of $25 \mu \mathrm{l}$ per reaction. The PCR products were run on an ethidium-bromide- 
containing $1 \%$ agarose gel with a size marker (Thermo Fischer Scientific). The primers for APP and PS1 are in the table below

\begin{tabular}{|c|c|c|}
\hline Name & \multicolumn{1}{c|}{ Sequence } & Product size (bp) \\
\hline PS1 For & CAG GTG CTA TAA GGT CAT CC & 291 \\
PS1 Rev & ATC ACA GCC AAG ATG AGC CA & 291 \\
APP For & GAA TTC CGA CAT GAC TCA GG & 246 \\
APP Rev & GTT CTG CTG CAT CTT GGA CA & 246 \\
\hline
\end{tabular}

\subsection{Cell culture and Exosomes}

\subsubsection{Primary neuron culture}

Neuronal cell culture, A-beta treatment and exosome isolation were performed in the laboratory of Dr Anja Schneider, at the Max Planck Institute for Experimental Medicine, Göttingen. Cells from the hippocampus or cerebral cortex of $\mathrm{C} 57 \mathrm{BI} / \mathrm{J}$ mouse embryos at day 16-18 were used to generate primary cultures. After euthanizing the pregnant female with carbon dioxide, the amniotic sac was dissected out and the embryos separated. After severing the head and separating the skull and meningeal coverings, the hippocampus or the cortex was dissected out under sterile Hank's buffered saline solution HBSS. After completing the dissection at room temperature, hippocampal or cortex tissue from all the embryos in the litter was placed in a $15 \mathrm{ml}$ tube with and $0.5 \mathrm{ml}$ of $0.05 \%$ trypsin-EDTA (Gibco), and incubated for 10 minutes at $37^{\circ} \mathrm{C}$. The trypsinization reaction was then stopped with Dubelco's Modified Eagle's Medium (DMEM) with $1 \%$ fetal bovine serum (PAA), 1\% Glutamax (Gibco), 2\% 50x B27 supplement (Gibco) and 1\% Pen Strep (Gibco) for 5 minutes at $37^{\circ} \mathrm{C}$, following which the mixture was pipetted up and through a glass pipette to obtain a single-cell suspension. After spinning at $1000 \mathrm{~g}$ at $4^{\circ} \mathrm{C}$ for 10 minutes, the cell pellet was suspended in $1 \mathrm{ml}$ neuronal growth medium (Modified Eagle's Medium (MEM), with $0.6 \%$ glucose, $0.2 \%$ sodium bicarbonate (Gibco), 1\% 100X sodium pyruvate (Gibco), 1\% fetal bovine serum (PAA), 1\% Glutamax (Gibco), 2\% 50x B27 supplement (Gibco) and 1\% Pen Strep (Gibco)).Viable neurons were counted using a Neubauer counting chamber after mixing $1 \mu \mathrm{l}$ of cell suspension with $1 \mu \mathrm{l}$ of trypan blue. The neurons were plated on $10-\mathrm{cm}$ dishes or in 12-well plates pre-coated with poly-L-lysine. The neurons were grown for up to 3 weeks in $10 \mathrm{ml}$ or $1 \mathrm{ml}$ of neuronal growth medium in $10 \mathrm{~cm}$ dishes or 12-well plates respectively.

Before exosome isolation, the neuronal growth medium was exchanged for a smaller volume of serum-free exosome collecting medium (Dubelco's Modified Eagle's Medium (DMEM) with 1\% Glutamax (Gibco), 2\% 50x B27 supplement (Gibco) and 1\% Pen Strep (Gibco)) and exosomes were collected for 1 hour, 6 hours, or overnight as required. The collecting medium was subjected to the exosome isolation steps below. After removing the collection medium for exosome isolation, the cell were washed once with phosphate-buffered saline (PBS) before adding $0.5 \mathrm{ml}$ of Trizol to the plate or $200 \mu \mathrm{l}$ of Trizol to the well. A cell scraper was then used to detach the cells from the surface and the cells were stored in Trizol until further processing for RNA isolation. 


\subsubsection{Amyloid-beta treatment}

The Amyloid-beta 42 (Aß42) oligomers and Amyloid-beta 4 (Aß40) monomers were obtained from the laboratory of Dr Markus Zweckstetter at the Max Planck Institute for Biophysical Chemistry, Göttingen. Oligomers or monomers were prepared in a stock solution of 100um in 10X phosphate buffered saline and used at a final concentration of $10 \mathrm{um}$. Primary hippocampal neurons in 10-cm dishes (4 dishes per condition) were treated with $A \beta 42$ oligomers, $A \beta 40$ monomers, and vehicle at day 15 in vitro After overnight treatment, one set of dishes was used for the MTT assay, and exosomes and cells from the remaining 3 dishes per condition were used for RNA isolation.

\section{MTT assay}

Thiazolyl Blue Tetrazolium Bromide (Sigma), at a concentration of $5 \mathrm{mg} / \mathrm{ml}$ in PBS was used for the MTT assay. After treatment with A-beta, the A-beta- or vehicle-containing medium was replaced with $3 \mathrm{ml}$ of fresh medium per $10-\mathrm{cm}$ dish and $300 \mu \mathrm{l}$ of MTT solution was added. After incubation for one hour at $37^{\circ} \mathrm{C}$, the medium was pipetted off and $3 \mathrm{ml}$ DMSO added. After shaking for 20 minutes at room temperature, $100 \mu \mathrm{l}$ of the solution was transferred to a 96-well plate in triplicate. An ELISA plate reader was used to quantify the assay colorimetrically at a wavelength of $570 \mathrm{~nm}$.

\subsubsection{Exosome Isolation}

Exosomes were isolated from fresh cell culture medium, or frozen cerebrospinal fluid or plasma. When isolating exosomes from cultured cells, cellular growth medium was exchanged for a lower volume of fresh 'collecting medium'. The cells were grown in the fresh medium for 1 hour, 6 hours or overnight, as required and this time was called 'collection time', after which the medium was harvested and used for exosome isolation.

The following protocol was used to isolate exosomes from cell culture medium, cerebrospinal fluid or plasma. The fluid was subjected to serial centrifugation at $3500 \mathrm{~g}$ for 10 minutes, $4500 \mathrm{~g}$ for 10 minutes, and $10000 \mathrm{~g}$ for 30 minutes, with the supernatant collected each time and pellet discarded. The $10000 \mathrm{~g}$ pellet from CSF and plasma was saved and processsed as the microvesicle fraction. Finally the supernatant was subjected to ultracentrifugation at $100000 \mathrm{~g}$ to obtain the exosome pellet and the exosome-free supernatant. The exosomes were resuspended/lysed in $200 \mu \mathrm{ITRI}$-reagent (Sigma) and stored at $-20^{\circ} \mathrm{C}$ until RNA isolation.

\subsection{Cerebrospinal fluid and plasma from patients}

Cerebrospinal fluid for exosome preparation was obtained from $18 \mathrm{AD}$ patients and 20 controls. The AD patients were all from the Göttingen University Hospital. Of the control samples, 14 were obtained from the same source and the remaining six were obtained through Dr Brit Mollenhauer from the Paracelsus Elena Clinic, Kassel. The control samples were from patients suffering from other neurological and psychiatric disorders. Analysis of patient CSF was approved by the ethical committee of the Medical Faculty, University Medicine Goettingen (IRB 02/05/09). 
Individual patient details and a summary of patient characteristics can be found in tables 1 and 2. After CSF withdrawal via lumbar puncture, the standard operating procedure was to centrifuge the sample as soon as possible at $3000 \mathrm{~g}$ for 10 minutes to remove any cells or cell debris. The supernatant was then frozen and saved at $-20^{\circ} \mathrm{C}$ until exosome isolation. In all cases a volume of $2 \mathrm{ml}$ of CSF or plasma was used for isolation of exosomes.

\subsection{RNA Isolation and profiling}

\subsubsection{RNA isolation from exosomes, tissues and cultured cells}

All RNA samples were isolated using TRI-Reagent (Sigma-Aldrich),except when otherwise specified. The sample (dissected tissue, or exosomes/microvesicle pellet) was thoroughly homogenized in $1 \mathrm{ml}$ TRIReagent and incubated at room temperature for 5 minutes. In cases where low RNA yield was expected, such as exosomes, microvesicles or volumes of tissue, $2 \mu \mathrm{l}$ of $25 \mathrm{ug} / \mathrm{ul}$ glycogen was added with trizol. 200 $\mu \mathrm{l}$ of chloroform was then added and mixed by vigorous shaking, followed by a second 5 -minute incubation step at room temperature. The mixture was centrifuged at 12000 rcf for 15 minutes until phase separation and the topmost aqueous RNA-containing layer pipetted into a new $1.5 \mathrm{ml}$ tube. The RNA was precipitated using $500 \mu \mathrm{l}$ of isopropanol overnight at $-20^{\circ} \mathrm{C}$. After centrifugation at $12000 \mathrm{rcf}$ for $30 \mathrm{~min}$ the supernatant was discarded and the pellet washed 2 times (12000 rcf for 5 minutes each) with $75 \%$ ethanol. The RNA pellet was air-dried and resuspended in 30-50 $\mu \mathrm{l}$ of water, depending on the expected RNA yield. For exosome isolation, the RNA was resuspended in $10 \mu$ of water.

RNA from brain tissue was quantified using a NanoDrop spectrophotometer, For RNA from exosomes, total Eukaryotic pico 6000 RNA assay (Agilent) and the corresponding RNA 6000 PicoChips were used to quantify RNA and study its profile on a 2100 Agilent Bioanalyzer microfluidics platform according to the manufacturer's instructions.

\subsection{2 cDNA synthesis and qPCR}

For microRNA real-time PCR, cDNA was synthesized using the miScript II RT kit (Qiagen) using the supplied protocol. The SYBR green-based miScript qPCR system (Qiagen) was used for real time PCR. Reactions were carried out in a reaction volume of $10 \mu$ using predesigned microRNA-specific assay primers (Qiagen) and cDNA at a dilution of 1:200. RNU6B was used as a reference small RNA and the standard amplification protocol for microRNA was denaturation at $95^{\circ} \mathrm{C}$ for 5 minutes, followed by 40 amplification cycles at $94^{\circ} \mathrm{C}$ for 15 seconds, $55^{\circ} \mathrm{C}$ for 30 seconds, $70^{\circ} \mathrm{C}$ for 30 seconds and a cooling step at $40^{\circ} \mathrm{C}$ for 30 seconds.

For mRNA real-time PCR, cDNA was synthesized using the Transcriptor First Strand cDNA synthesis kit (Roche). The TaqMan reactions were carried out in a volume of $25 \mu$ using selected automatically designed primers from the Universal ProbeLibrary (UPL) assay design center, the corresponding UPL fluorescent probes (Roche), LightCycler 480 master mix and cDNA at a dilution of 1:5. HPRT1 (mouse) or GAPDH (human) was used as a reference gene and the standard amplification protocol was denaturation at $95^{\circ} \mathrm{C}$ for 5 minutes, followed by 40 amplification cycles at $95^{\circ} \mathrm{C}$ for 10 seconds, $60^{\circ} \mathrm{C}$ for 30 seconds, $72^{\circ} \mathrm{C}$ for 1 second and a cooling step at $40^{\circ} \mathrm{C}$ for 30 seconds. All qPCR reactions were carried out in a LightCycler 480 instrument (Roche), analyzed using the accompanying LightCycler 480 software with the delta-delta 
Ct method used for quantification.

\section{Library preparation}

Small RNA libraries were prepared from total RNA using the TruSeq small RNA sample preparation kit (Illumina) according to the manufacturer's instructions. When relatively plentiful amounts of RNA were available as in the case of hippocampal tissue, $100 \mathrm{ng}$ of total RNA was used as starting material. In the case of exosomal RNA, lower amounts were used, as described in detail in section 3.2.5.

\subsection{Bioinformatics and statistical analysis}

\subsubsection{Primary and secondary analysis of next-generation sequencing data}

The processing of data produced by the HiSeq sequencer up to the generation of mRNA or non-coding RNA reads was performed by the group of Dr Stefan Bonn at the NGS facility of the DZNE, Göttingen. A customized software pipeline was used, consisting of the following steps. For mRNA, the CASAVA 1.8.2 software (Illumina) was used for demultiplexing and quality control, followed by BOWTIE2 for alignment to the human or mouse genome [212], and HTSeq for read counting. For microRNA and other non-coding RNAs, after demultiplexing and quality control, the basic local alignment search tool (BLAST) [213] was used for alignment to MiRBase version 19, with a cutoff score of $5 \times 10^{-7}$ and single mismatch allowed. The remaining reads were mapped to the non-coding RNA database fRNAdb version 3.4.

\subsubsection{Statistical and functional analysis of next-generation sequencing data}

The $R$ environment, and various user-contributed $R$ packages was used for statistical analysis and visualization of the read count data sets, as detailed below. The DESeq package [214] was used to obtain lists of mRNA or microRNAs differentially expressed between groups. Heatmaps were generated using the ggplot2 package. The machine learning algorithms Random Forest and Support Vector Machines were implemented using the $\mathrm{R}$ packages randomForest and e1071 respectively.

Microsoft Excel was used to calculate Pearson correlations and $p$ values for matrices of gene and microRNA expression levels in individual mice. Graphpad prism version 6 was used for analysis and visualization of behavior data, GPCR results, and individual RNA read count correlations. The GSEA algorithm was implemented using the desktop Java application javaGSEA. A list of gene symbols and corresponding correlation $p$ values was submitted in the required .rnk format to the GseaPreranked tool. For gene ontology (GO) analysis, the GO gene set collection (c5) from the Molecular Signatures Database version 4.0 was used. For analysis of Kyoto Encyclopedia of Genes and Genomes (KEGG) pathways the curated KEGG collection (c2) was used, from the same database. The entire list of GO categories obtained by GSEA with the corresponding false discovery rates were submitted to the REVIGO online tool to create a visualization of the over-represented GO terms [215]. 
Methods and Materials 

3. Results 



\subsection{Age-associated memory impairment}

The degree of age-associated memory impairment varies highly between individuals. Thus within a group of aging people, there are some who retain good cognitive skills, while others show a decline. One of the aims of the work described below was to test if this intra-group homogeneity and its molecular correlations could be modeled in mice. This would allow us to differentiate between general age-associated changes in the brain and age-related changes that have a connection with memory impairment.

\subsubsection{Aging is associated with increased variability in water maze task performance}

In order to study the effects of age on intra-group variability in mice, individually housed and identically treated C57BI6J mice at the ages of 3 months (young adult, $n=12$ ), and 16 months (early aging, $n=12$ ) were trained in the hippocampus-dependent Morris water maze memory task.

Learning and memory performance was analyzed in different groups of mice over a period of 10 days. As expected, the old mice showed a learning impairment, indicated by increased escape latency or time required to reach the hidden platform (Fig 3.1.1A). When the performance of mice in both groups is plotted individually (Fig 3.1.1B) a marked difference in variability within groups is evident. While the young mice cluster tightly together by performance, individuals within the group of old mice perform very differently from one another. The group of old mice is thus composed of individuals that are able to perform on par with the young mice as well as individuals with varying degrees of impairment. This variability was quantified using the $\mathrm{F}$ test for equality of variance, confirming that for most of the tested days the 16-month-old group showed a significantly higher intra-group variance than the 3-month-old group.

To demonstrate that variability increases progressively with age, additional groups of mice at the ages 8 months (adult, $\mathrm{n}=12$ ) and 12 months (middle-aged, $\mathrm{n}=12$ ) were trained in the water maze task for 4 days, and a cross section of WM escape latency in these 4 groups is depicted in (Fig 3.1.1 C). Again, when plotted individually, an age-dependent increase in variability within groups is observed, and quantified using the $\mathrm{F}$ test for equality of variance (Fig 3.1.1D).

\subsubsection{No increase in variability on other learning tasks, visual or motor skills}

The 3- and 16-month old groups of mice were subjected to a battery of additional behavior tests to study impairment and variability on other cognitive measures. When performance was tested by the probe test, a test for long term spatial recall, carried out on the last day of the water maze task, the young mice spent significantly more time than the old mice in the target quadrant, where the platform had been placed over the training period (Fig 3.1.2A). Though the old mice were significantly impaired, there did not appear to be a difference in variability between the 2 groups (Fig 3.1.2A). Similar results were observed on the fear conditioning test, an associative memory test, where the mice are trained to associate an aversive stimulus with a given context. The young mice displayed a significantly higher level of freezing than the old mice when placed back in the context (Fig 3.1.2B), indicating that the development of associative fear memory was impaired in the old mice. As with the probe test, there was no difference in variability between the groups (Fig 3.1.2B). The third behavior test performed on these groups was the novel object 
A

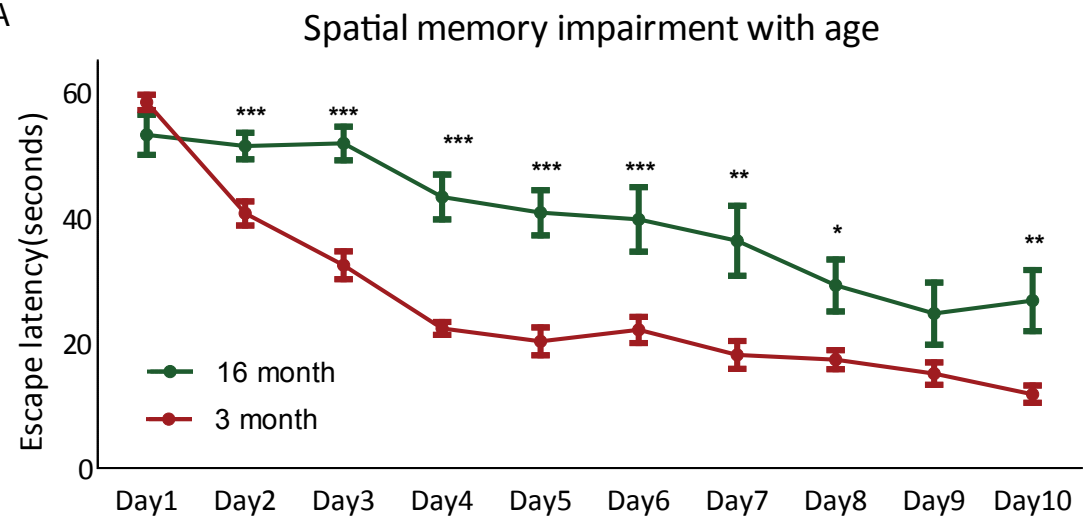

B

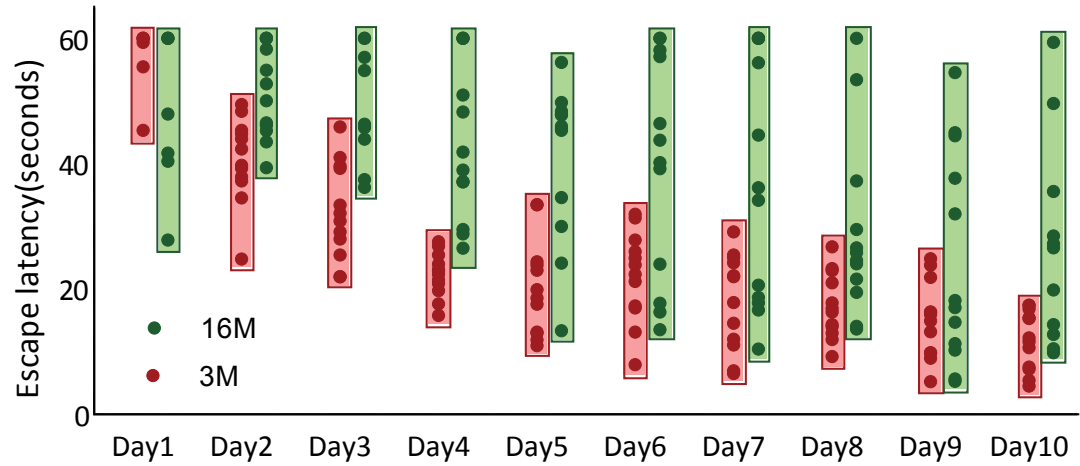

C Day 4 Escape Latency

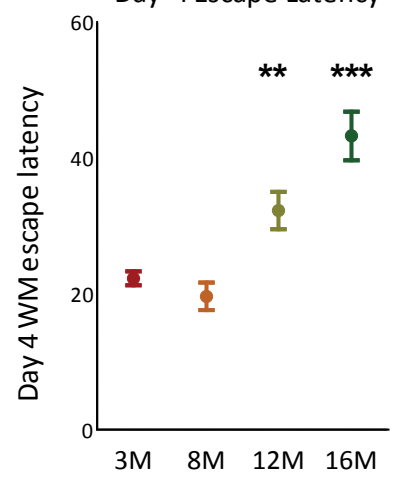

Day 4 EL Variability

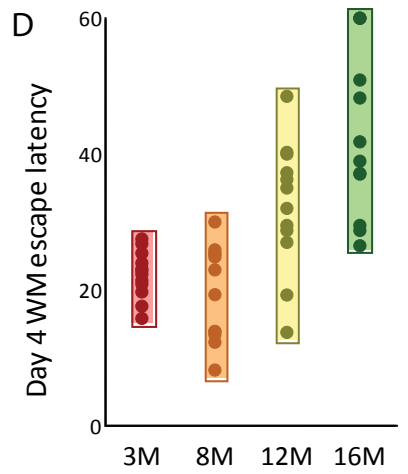

Figure 3.1.1:Aging is associated with increased variability in water maze task performance

A: Group performance of old(16-month-old) and young(3-month-old) mice on the training days of the Morris water maze task. $(\mathrm{N}=12$ per group, student's two-tailed $\mathrm{T}$ test $\mathrm{p}$ value $=0.0011,<0.0001,<0.0001$, $0.0044,0.0061,0.0138,0.0823$, and 0.0059 on days $2,4,5,6,7,8,9$ and 10 respectively), B: Individual performance of old(16-month-old) and young(3-month-old) mice on the training days of the Morris water maze task $(\mathrm{N}=12$ per group, $\mathrm{F}$ test for equality of variances, $\mathrm{p}$ value $=0.0041,0.8037,0.0002,0.0057$, $0.0048,0.0023,0.0019$ and 0.0004 on days $1,2,4,6,7,8,9$ and 10 respectively), C: Cross section on day 4 of the water maze test, showing group performance at the age of $3,8,12$ and 16 months $(n=12$ per group, student's T test $p$ values: 3 versus 12 months $=0.0025$ and 3 versus 16 months $<0.0001) \mathrm{D}$ : Cross section on day 4 of the water maze test, showing individual performance at the age of 3,8,12 and 16 months ( $n=12$ per group, $F$ test for equality of variances $p$ values: 3 versus 8 months $=0.03,3$ versus 12 months $=0.0025$ and 3 versus 16 months $=0.0002$ ) 

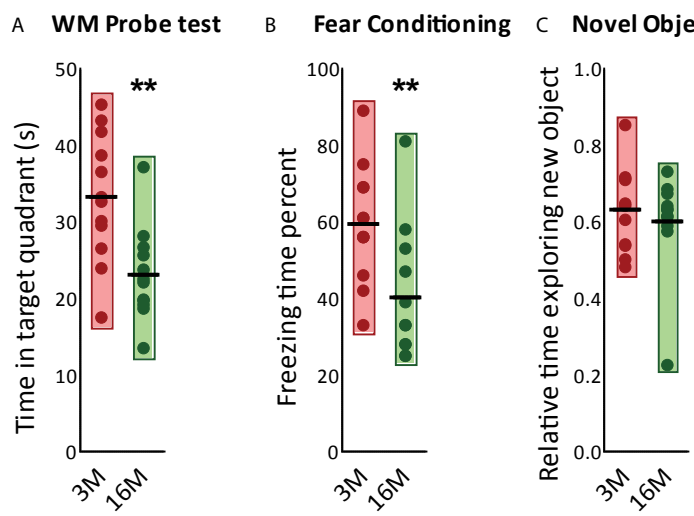

D Novel Object LTM

E Visible Platform

F Visible Platform

G Rotarod
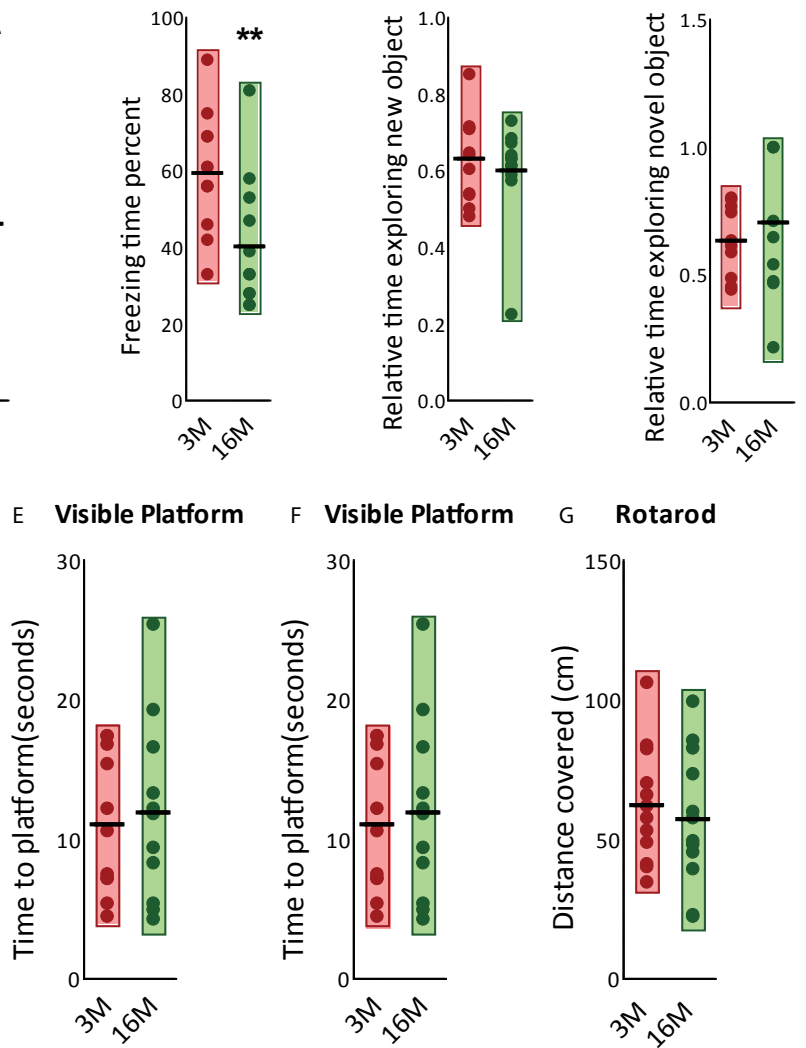

Figure 3.1.2 No increase in variability on other learning tasks, visual or motor skills

Other behavior tests :Performance of 3- and 16-month old mice( $n=12$ per group) on the water maze probe test(A),Fear conditioning task(B),novel object recognition task-short term memory $(C)$ and novel object recognition task-short term memory (D). Each circle represents an individual animal. Mean is represented by a black horizontal line. (student's two-tailed T test $p$ value $=0.0077$ for probe test and 0.0023 for fear conditioning task.)

Visual and motor skills: Performance of 3- and 16-month old mice( $n=12$ per group) on the visible platform probe test $(E)$,swim speed(F), and rotarod test $(G)$. Each circle represents an individual animal. Mean is represented by a black horizontal line 
recognition test, which evaluates the ability to learn and remember objects previously explored. This test was performed to test both long-term and short term memory in the 2 groups of mice. The readout for the test is the time spent exploring the novel object, which did not differ significantly between groups, either long-term or short term (Fig 3.1.2 C and 3.1.2D). Similarly there was no difference in variability between groups (Fig 3.1.2C and 3.1.2D).

Since aging is accompanied by motor and visual deficits which could potentially influence the effects of the learning and memory tests 3 tests for sensorimotor skills were carried out in these 2 groups of animals. Two of these tests were within the context of the water maze task - the visible platform probe test, where the underwater platform is flagged tests for visual impairment, and the swim speed test detects difficulties in swimming that could hamper water maze performance. The third test was the rotarod test, which tests balance, coordination, motor planning and general physical condition. On all three tests, the aged group performed no differently than the young group (Fig 3.1.2E, $F$ and $G$ ) and there was no difference on variability between groups, indicating that at 16 months of age, the mice show no motor or visual deficits that could account for impaired performance on the cognitive behavior tests.

\subsubsection{Individual performance on behavior tests is consistent}

\subsubsection{Individual performance on the water maze task is consistent}

Since mouse performance varies within the group of animals at the same age, especially for aged mice, the question that arises is how consistent are the aged and young mice in their learning abilities from day to day. Are the mice that find the platform quickly on the first day of water maze training the same ones that do so on day 2, day 3 and the other testing and training days? Similarly, in the group of old mice, are the impaired learners impaired throughout the duration of the task, or are there high inter-day fluctuations in individual performance? In order to answer these questions, correlations between water maze performance through the various days of the task were measured in groups of male mice at the ages of 3 months $(n=$ $30), 12$ months $(n=23)$ and 16 months $(n=23)$ (Fig 3.1.3).

For each age group, correlations between escape latency on days 2,3, and 4 of the task were measured and are displayed as scatter plots (Fig 3.1.3A, B and C). For the group at 3 months of age, data are also available for days 5 and 6 (Fig 3.1.3 D). An additional measure of water maze spatial learning is the probe test, and the readout for the probe test is time spent in the platform quadrant. For the probe test, better performance is gauged by longer time spent looking in the correct quadrant. Correlations were also measured between probe test performance and average performance throughout the water maze training days as well as between probe test performance and water maze escape latency on the last day (day before probe test) (Fig 3.1.3D). The squared Pearson's correlation coefficient $\left(R^{2}\right)$ and the corresponding $p$ value are used to quantify the correlation. From the tables in Fig 3.1.3, it is clear that for almost all the days tested and at all ages, there exist statistically significant positive correlations in mouse performance between days. In case of the probe test, the correlations are negative (negative $R$ values), meaning that longer time searching in the correct quadrant during the probe test correlated with short escape latencies on the training days.

For each tested day and for the probe test, correlations were also measured between water maze escape 
A

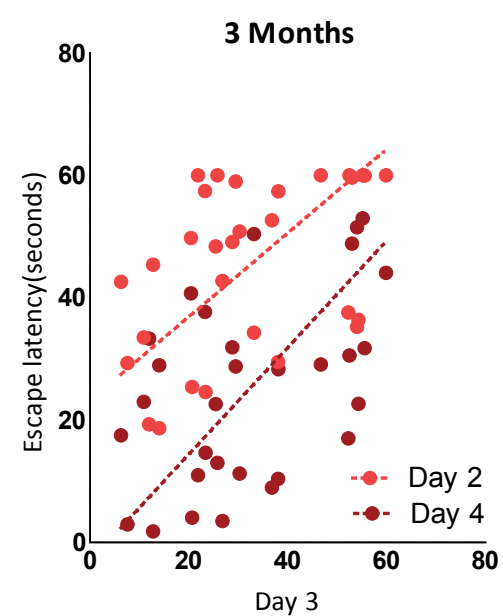

$B$

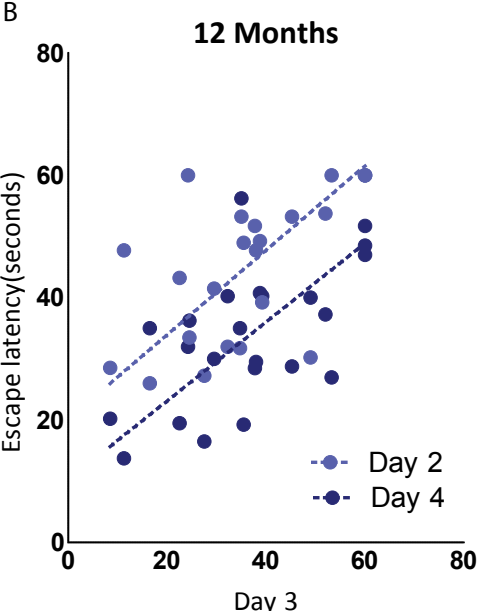

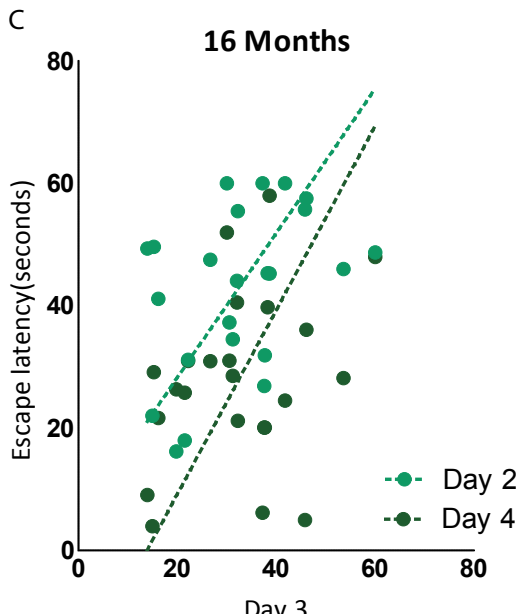

3 Months

Water maze days

\begin{tabular}{|llrr|}
\hline WM parameter 1 & WM parameter 2 & \multicolumn{1}{c}{ R } & P value \\
\hline Day 2 & Day 3 & 0.447213595 & 0.0122 \\
Day 3 & Day 4 & 0.489897949 & 0.0058 \\
Day 4 & Day 5 & 0.1 & 0.5887 \\
Day 5 & Day 6 & 0.424264069 & 0.0176 \\
Day 4 & Day 6 & 0.509901951 & 0.0037 \\
All days avg & Probe test & -0.63245553 & 0.0002 \\
Last day & Probe test & -0.50990195 & 0.0037 \\
\hline
\end{tabular}

12 Months

Water maze days

\begin{tabular}{|llcr|}
\hline WM parameter 1 & WM parameter 2 & \multicolumn{1}{c}{ R } & P value \\
\hline Day 2 & Day 3 & 0.519615242 & 0.0035 \\
Day 3 & Day 4 & 0.533385414 & 0.0029 \\
All days avg & Probe test & -0.33301652 & 0.0775 \\
Last day & Probe test & -0.25859234 & 0.1756 \\
\hline
\end{tabular}

16 Months

Water maze days

\begin{tabular}{|llcr|}
\hline WM parameter 1 & WM parameter 2 & \multicolumn{1}{c}{ R } & P value \\
\hline Day 2 & Day 3 & 0.423438307 & 0.0441 \\
Day 3 & Day 4 & 0.300449663 & 0.1636 \\
All days avg & Probe test & -0.24409015 & 0.2617 \\
Last day & Probe test & -0.53935146 & 0.0079 \\
\hline
\end{tabular}

\begin{tabular}{|llll|}
\hline Day 2 & Swim speed & 0.172133669 & 0.3631 \\
Day 3 & Swim speed & 0.075544689 & 0.6915 \\
Day 4 & Swim speed & 0.148256534 & 0.4343 \\
Day 5 & Swim speed & 0.110679718 & 0.5604 \\
Day 6 & Swim speed & $8.48 E-003$ & 0.9645 \\
All days avg & Swim speed & 0.16087262 & 0.3958 \\
Probe test & Swim speed & 0.148256534 & 0.4343 \\
\hline
\end{tabular}

Swim speed

\begin{tabular}{|llll|}
\hline Day 2 & Swim speed & 0.041243181 & 0.8318 \\
Day 3 & Swim speed & 0.177932571 & 0.3557 \\
Day 4 & Swim speed & 0.083677954 & 0.6661 \\
All days avg & Swim speed & 0.333016516 & 0.0775 \\
Probe test & Swim speed & 0.229651911 & 0.2308 \\
\hline
\end{tabular}

Swim speed

\begin{tabular}{|lllr|}
\hline Day 2 & Swim speed & 0.056973678 & 0.7962 \\
Day 3 & Swim speed & 0.050705029 & 0.8183 \\
Day 4 & Swim speed & 0.37469988 & 0.0782 \\
All days avg & Swim speed & 0.391152144 & 0.065 \\
Probe test & Swim speed & 0.358887169 & 0.0927 \\
\hline
\end{tabular}

Figure 3.1.3.1 Individual performance on the water maze task is consistent

A: Correlations between water maze escape latency on day 3 ( horizontal axis) and water maze escape latency on days 2 (light circles) or 4 (dark circles) in groups of mice at the ages of 3 months $(A, n=30), 12$ months $(B, n=23)$, and 16 months $(C, n=23)$. Deming regression lines are indicated on each plot and each solid circle represents an individual animal., D: Tables showing correlation between water maze paramaters on various days in groups of mice at the ages of 3 months $(n=30), 12$ months( $n=23)$, and 16 months ( $=23$ ). Statistically significant $\mathrm{p}$ values shaded in grey, E: Tables showing correlation between water maze parameters and swim speed in groups of mice at the ages of 3 months $(n=30), 12$ months $(n=23)$, and 16 months $(n=23)$ 
latency or time in target quadrant and individual swim speed (Fig 3.1.3E). There were no correlations either positive or negative between swim speed and the indexes of water maze spatial learning at any age (Fig3.1.3E). This finding shows once again that water maze performance at the ages tested is not influenced by sensorimotor abilities or impairment.

Thus the repeated finding in various age groups on water maze task performance allows us to conclude that the water maze task can be reliably used to distinguish aged individuals with impaired spatial learning ability from those who retain the ability to learn and recall spatial information even as they age.

\subsubsection{Individual performance on the fear conditioning task is consistent}

After establishing for the water maze spatial learning task that distinct 'good learners' and 'poor learners' exist within the group of animals at the same age, we checked if similar correlations were present in other tests of mouse learning and memory - fear conditioning extinction and the novel object recognition task.

The fear conditioning extinction protocol, performed on the days immediately following the fear conditioning task, consists of placing the mice back in the fear-associated context for 3 minutes each day over several days. It can be observed that normal individuals will show less freezing behavior as the days pass, a phenomenon that is called fear extinction [216]. This behavior has been attributed to 'new learning', where the mice acquire a competing memory that the context is safe, and to habituation to the context [216]. Both the fear conditioning task and the fear extinction protocol use freezing behavior or percentage of time spent freezing as their readout. In a group of mice at the age of 3 months $(n=30)$ correlations between freezing behavior and extinction behavior were measured over 4 days after fear conditioning (Fig 3.1.3.2 $A$ and $B$ ). After the aversive stimulus, there are high individual correlations in time spent freezing on the fear conditioning test day and through the extinction days. The mice that spend the longest time freezing when tested in the context 24 hours after the aversive stimulus tend to show a slower extinction of this fear memory and continue to show high freezing throughout the extinction protocol. Further, at both 3 months and 12 months of age, prestimulus freezing or baseline freezing before exposure to the aversive stimulus show correlations with post-stimulus freezing behavior.

\subsubsection{No correlations exist between performance on different memory tasks}

The water maze task and the fear extinction protocol are multi-day tests, which lend themselves very well to the analysis of individual performance at different time points. The third test performed on the groups of animals at the ages of $3(n=30)$ and 12 months $(n=23)$, the novel object recognition task studies exploratory behavior, short term memory and long term memory.

In order to compare individual performance on all these 3 tests, correlations in inter-individual performance between the 3 different tests were measured. (Fig 3.1.3.3). There were no correlations between performance on water maze and performance on either fear conditioning or novel object recognition. Similarly, no correlations between the fear conditioning and novel object recognition task performance were observed (Fig 3.1.3.3). These results suggest that performance on one memory test is not predictive of performance on other tests and that impairment in the learning mechanisms involved in spatial, associative and other types of learning could be independent. 
Overall, at the early-aging time point of 16 months, since the water maze task showed the widest spectrum of intra-group variability (Fig 3.1.1) and seemed a robust method to distinguish between poor and good learners (Fig 3.1.3). Therefore this task was chosen for further investigation into the molecular mechanisms underlying the variability in age-related learning and memory impairment.

\subsubsection{Gene expression in aging mice}

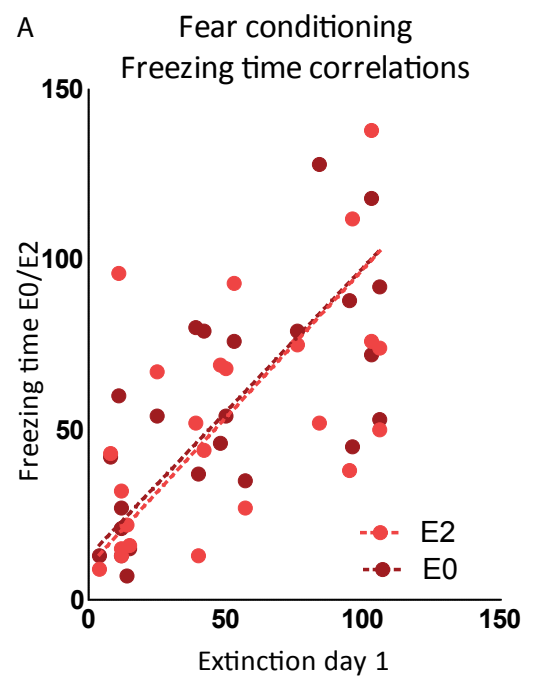

B

Fear conditioning - Freezing time correlations

\begin{tabular}{|llllr|}
\hline Age & \multicolumn{1}{c}{ Freezing time X } & Freezing time Y & \multicolumn{1}{c|}{ R } & P value \\
\hline 3M & Poststim Day 0 & Poststim Day 1 & 0.698498389 & 0.0001 \\
3M & Poststim Day 0 & Poststim Day 2 & 0.596070466 & 0.0021 \\
3M & Poststim Day 0 & Poststim Day 3 & 0.693397433 & 0.0002 \\
3M & Poststim Day 0 & Poststim Day 4 & 0.318433667 & 0.1294 \\
3M & Prestim & Poststim Day 0 & 0.280570847 & 0.1842 \\
3M & Prestim & Poststim Day 1 & 0.301628911 & 0.152 \\
3M & Prestim & Poststim Day 2 & 0.523259018 & 0.0087 \\
3M & Prestim & Poststim Day 3 & 0.05205766 & 0.8091 \\
3M & Prestim & Poststim Day 4 & 0.122882057 & 0.5673 \\
12M & Prestim & Poststim Day 1 & 0.517300686 & 0.0041 \\
\hline
\end{tabular}

Figure 3.1.3.2 Individual performance on the fear conditioning task is consistent

A: Correlations between time spent freezing on the first day of exctinction (horizontal axis) and time spent freezing when testing fear memory (light circles) or on the second day of exctinction (dark circles) in a group of 30 mice at the age of 3 months $(A, n=30)$. Deming regression lines are indicated and each solid circle represents an individual animal, B: Tables showing correlation between the pre-stimulus and poststimulus freezing behavior in mice at the ages of 3 months $(n=30)$ and 12 months $(n=23)$. 

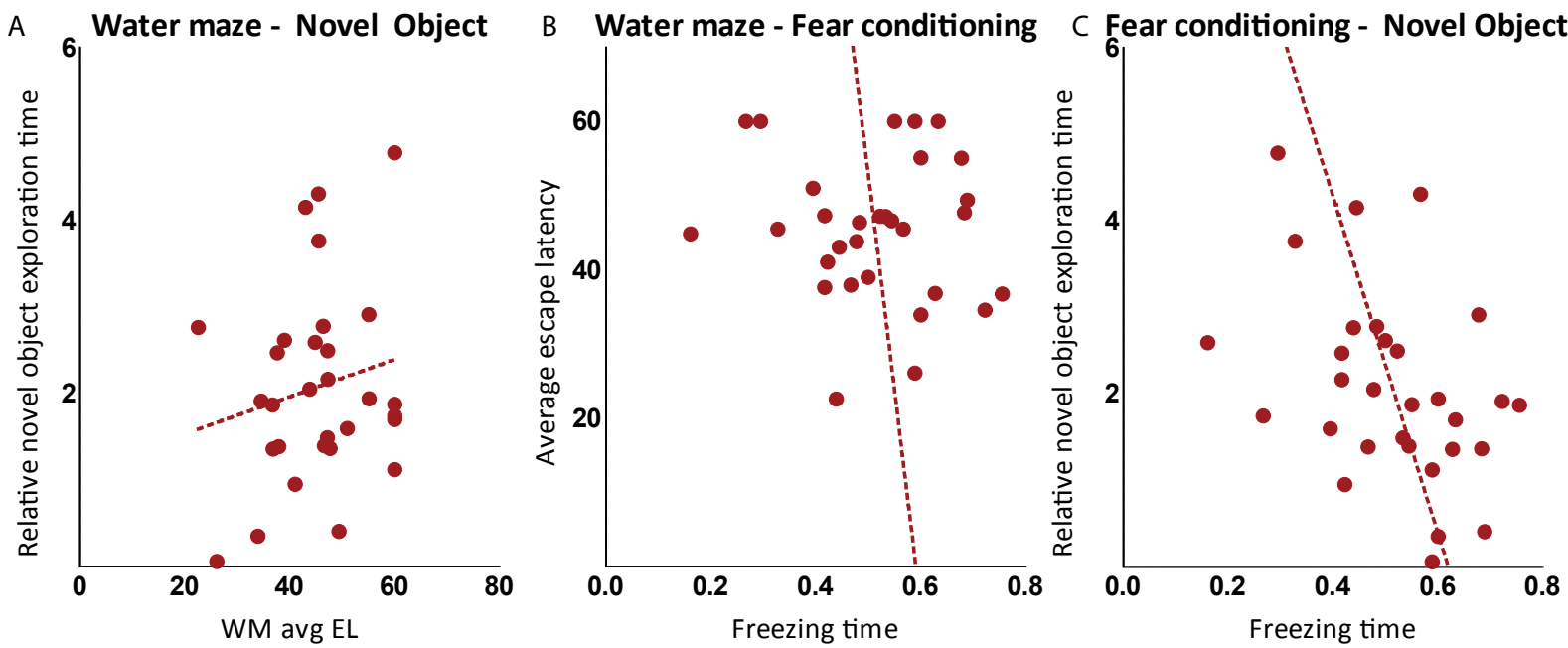

D Water maze - Novel Object

\begin{tabular}{|lllrr|}
\hline Age & Water Maze & Novel object & \multicolumn{1}{c|}{ R } & \multicolumn{1}{l|}{ P value } \\
\hline 12M & Avg escape latency & Novel Object time & 0.1624 & 0.4 \\
12M & Probe test & Novel Object time & -0.21 & 0.2601 \\
3M & Average Escape latency & Novel Object time & 0.1849 & 0.3279 \\
3M & Probe test & Novel Object time & -0.2272 & 0.2272 \\
\hline
\end{tabular}

E Water maze - Freezing time

\begin{tabular}{|lllcr|}
\hline Age & Water Maze & Freezing time & R & P value \\
\hline $12 \mathrm{M}$ & Avg escape latency & Freezing time & 0.119749739 & 0.5361 \\
12M & Probe test & Freezing time & 0.351710108 & 0.0614 \\
3M & Average Escape latency & Freezing time & 0.120083304 & 0.5273 \\
3M & Probe test & Freezing time & 0.197888858 & 0.2945 \\
\hline
\end{tabular}

F Novel Object - Freezing time

\begin{tabular}{|lllrr|}
\hline Age & \multicolumn{1}{c}{ Novel Object } & \multicolumn{1}{c}{ Freezing time } & \multicolumn{1}{c|}{ P value } \\
\hline $3 \mathrm{M}$ & Novel Object time & Freezing time & 0.407676342 & 0.0253 \\
$3 \mathrm{M}$ & Total exploration time & Freezing time & 0.31685959 & 0.0879 \\
$12 \mathrm{M}$ & Novel Object time & Freezing time & 0.166282891 & 0.3886 \\
$12 \mathrm{M}$ & Total exploration time & Freezing time & 0.365786823 & 0.051 \\
\hline
\end{tabular}

Figure 3.1.3.3 No correlations exist between performance on different memory tasks

Legend: A: Correlation between relative time spent exploring novel object in the novel object exploration task (horizontal axis) and average water maze escape latency over all tested days (vertical axis) B: Correlation between freezing time as a percentage of total time (horizontal axis) and average water maze escape latency over all tested days (vertical axis) C: Correlation between freezing time as a percentage of total time (horizontal axis)and relative time spent exploring novel object (vertical axis). Deming regression lines are indicated and each solid circle represents an individual animal, $n=30$, age $=3$ months). Tables showing correlation between parameters on the water maze and fear conditoning (D), water maze and novel object recognition tasks $(E)$ and fear conditioning and novel object recognition tasks $(F)$ in mice at the ages of 3 months $(n=30)$ and 12 months $(n=23)$. 
Having established that the water maze task can be used to reproducibly estimate the level of learning impairment in aging mice, the next step was to study the molecular changes associated with this impairment. Since the hippocampus is closely connected with both spatial learning and memory formation (See section 1.1.1), the method chosen for this was transcriptome and microRNAome profiling of the hippocampus.

A group of 22 mice at the age of 16 months was subjected to the water maze task for 4 days and the probe test was performed on day 5 . Mouse behavior was scored by performance on the probe test of the water maze. On this test, the time spent searching in the correct quadrant is an index of how well the mice were able to learn the task, with higher time spent indicating stronger learning. After confirming that there were significant correlations in inter-day performance within the task, the mice were sacrificed 2 weeks after the day of the probe test. Next generation sequencing was performed on RNA isolated from the hippocampi of these mice. Since the behavioral performance of the mice lies along a spectrum, each mouse was represented as an individual and tissue and RNA processing and data analysis were performed accordingly. Hippocampal gene expression was then correlated with individual performance on the water maze probe test (Fig3.1.4). In order to perform this analysis, Pearson product-moment correlation coefficients were calculated between normalized gene expression counts for all expressed genes and probe test performance of the individual. Of all the expressed genes, 765 showed a significant positive correlation with performance and 391 showed a significant negative correlation (Fig 3.1.4C and D) with performance (unadjusted $p$ values $<0.05$ ).

\subsubsection{Gene sets that correlate with memory performance}

In order to derive biological meaning from the gene lists obtained by correlating behavioral data with gene expression data, the gene set enrichment analysis (GSEA) method was used. GSEA is a computational tool initially developed for the analysis of microarray data when genome-wide expression profiling started to gain popularity [217]. The traditional method of extracting biologically relevant information from expression data involves choosing a differential expression fold change value and/or a $p$ value as a cutoff point for preparing a shortlist of genes. This shortlist, with all genes weighted equally regardless of original $p$ values or fold change, is then submitted to a tool that detects significant biological pathways or ontologies. In contrast, the GSEA algorithm is designed to analyze entire sets of genes associated with biological processes. GSEA is typically used on the complete genome-wide expression data, and the genes are weighted by their degree of dependence on the biological process being analyzed, such as by level of fold change. The algorithm then calculates enrichment scores for gene sets, based on the ranking of genes within that gene set in the experimental data. The level of statistical significance of the gene set is then calculated and adjusted for multiple testing [217]. This powerful analytical method is now being used for gene expression data obtained by NGS [218].

GSEA is particularly well suited to the analysis of the gene expression in the aging mice used here for two reasons; first - the correlation analysis used to obtain lists of important genes provides no fold-change values and second - using a cutoff of 0.05 on $p$ values after adjusting for multiple testing leaves too few genes for a meaningful analysis. The nature of the experiment, which aims to detect small gene expression changes in genetically-identical individuals within a group of animals at the same age thus necessitates a highly sensitive analysis approach. 

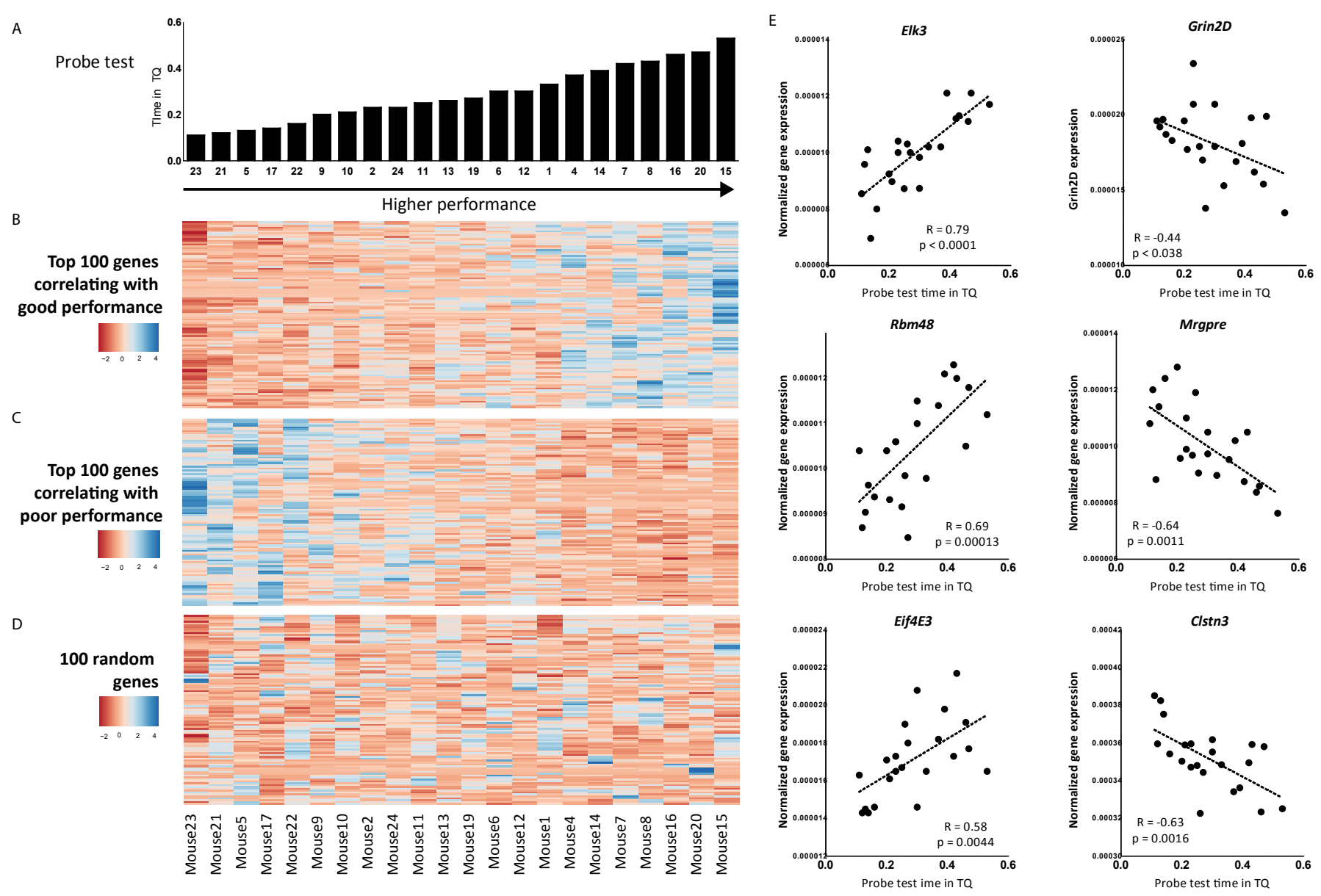

Figure 3.1.4 Gene expression in 16-month old mice

A; Time spent in target quadrant by 16 -month-old mice $(n=22)$, plotted individually and sorted from worst performace to best performance, Heat maps showing the scaled, normalized expression values of the 100 genes most significantly correlated positively $(B)$ and negatively $(C)$ with performance on the probe test. Color scale represent levels of expression, with blue representing highest expression and red representing the lowest expression, D: Heat map showing expression values of 100 randomly selected genes, E. Examples of correlations between performance and gene expression for the genes ETS domain-containing protein-3(Elk3), RNA binding motif protein-48(Rbm48), and Eukaryotic Translation Initiation Factor 4E3 (Eif4E3) which correlate positively with performance, and Glutamate receptor subunit epsilon-4 (Grin2D), Mas-related G-protein coupled receptor member E(Mrgpre) and Calsyntenin3 (Clstn3) whose expression correlates negatively with performance. The gene expression levels are plotted as normalized read counts on the vertical axis versus the probe test performance (percent time spent in target quadrant) on the horizontal axis. Deming regression lines are indicated and each solid circle represents an individual animal, $(n=22)$.

\section{Figure 3.1.5 Gene sets that correlate with memory performance (Facing Page)}

A: Graph showing number of GO-terms and KEGG pathways significantly enriched in the original correlation dataset (Red) and after 3 independent permutations of the data set (black). B: Tables showing enriched GOterms and KEGG pathways C: Graphical representation generated by the REVIGO tool of the molecular function $\mathrm{GO}$ categories. The enriched GO terms are represented on the graph as circles, with the most signifcantly enriched terms labeled. The color of cirlces represents enrichment scores, with red represeinting the most enirched in the positive direction and blue representing the most enriched in the negative direction. The horizontal axis also represents enrichment score and terms are organized vertically by their semantic similarity or degree of relationship. 


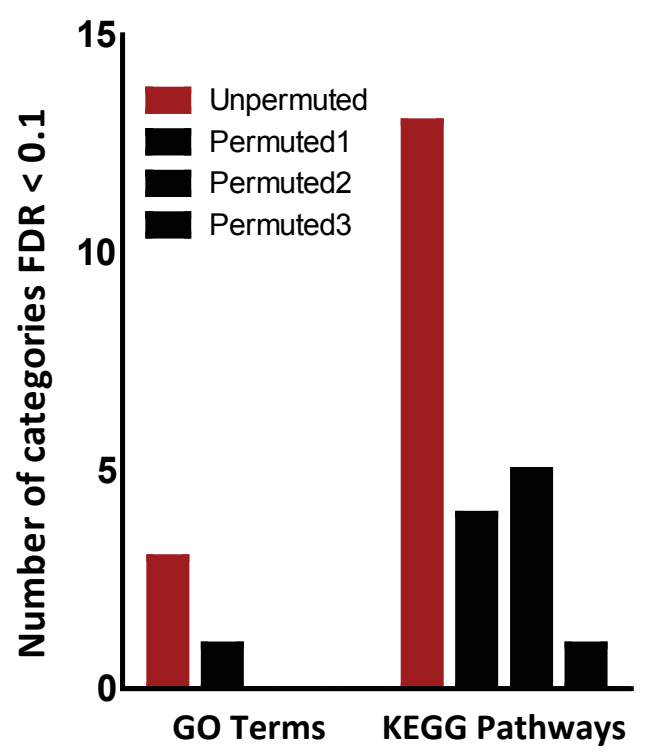

GO categories correlated with good performance

\begin{tabular}{|l|r|r|r|}
\hline NAME & Genes & NOM p-val & FDR q-val \\
\hline STRUCTURAL_CONSTITUENT_OF_RIBOSOME & 79 & $<0.0001$ & $<0.0001$ \\
TRANSLATION & 168 & $<0.0001$ & 0.08 \\
MITOCHONDRIAL_MEMBRANE & 84 & $<0.0001$ & 0.08 \\
RNA_BINDING & 247 & $<0.0001$ & 0.08 \\
MITOCHONDRIAL_MEMBRANE_PART & 51 & $<0.0001$ & 0.09 \\
CELLULAR_RESPIRATION & 19 & $1.75 E-003$ & 0.09 \\
BIOSYNTHETIC_PROCESS & 443 & $<0.0001$ & 0.1 \\
\hline
\end{tabular}

GO categories correlated with poor performance

\begin{tabular}{|l|r|r|r|}
\hline NAME & Genes & \multicolumn{1}{l|}{ NOM p-val } & \multicolumn{1}{l|}{ FDR q-val } \\
\hline GLUTAMATE_SIGNALING_PATHWAY & 17 & $2.52 \mathrm{E}-003$ & 0.01 \\
GLUTAMATE_RECEPTOR_ACTIVITY & 20 & $<0.0001$ & 0.02 \\
\hline
\end{tabular}

KEGG pathways correlated with good performance

\begin{tabular}{|c|c|c|c|}
\hline NAME & Genes & NOM p-val & FDR q-val \\
\hline KEGG_RIBOSOME & 85 & $<0.0001$ & $<0.0001$ \\
\hline KEGG_OXIDATIVE_PHOSPHORYLATION & 111 & $<0.0001$ & $6.25 \mathrm{E}-004$ \\
\hline KEGG_PATHOGENIC_ESCHERICHIA_COLI_INFECTION & 50 & $<0.0001$ & $2.52 E-003$ \\
\hline KEGG_PARKINSONS_DISEASE & 108 & $<0.0001$ & $1.61 E-002$ \\
\hline KEGG_PROTEIN_EXPORT & 22 & 9.95E-003 & 4.27E-002 \\
\hline KEGG_SNARE_INTERACTIONS_IN_VESICULAR_TRANSPORT & 33 & $<0.0001$ & 4.54E-002 \\
\hline KEGG_B_CELL_RECEPTOR_SIGNALING_PATHWAY & 72 & $3.00 E-003$ & 4.79E-002 \\
\hline KEGG_NOD_LIKE_RECEPTOR_SIGNALING_PATHWAY & 54 & $<0.0001$ & 4.88E-002 \\
\hline KEGG_VEGF_SIGNALING_PATHWAY & 70 & $<0.0001$ & $5.47 \mathrm{E}-002$ \\
\hline KEGG_FC_EPSILON_RI_SIGNAUNG_PATHWAY & 73 & $7.42 \mathrm{E}-003$ & 6.97E-002 \\
\hline KEGG_VIBRIO_CHOLERAE_INFECTION & 52 & $6.17 \mathrm{E}-003$ & $8.26 \mathrm{E}-002$ \\
\hline KEGG_RNA_POLYMERASE & 27 & $1.91 E-002$ & $8.79 \mathrm{E}-002$ \\
\hline KEGG_PROTEASOME & 43 & $1.77 \mathrm{E}-002$ & $9.27 \mathrm{E}-002$ \\
\hline
\end{tabular}


The entire list of genes expressed in the 16-month-old mice, ranked by correlation score was analyzed by GSEA, for enriched gene ontology (GO) terms and Kyoto Encyclopedia of Genes and Genomes (KEGG) pathways. GO terms provide information about the molecular functions and cellular compartments of the gene expression products [219] while KEGG pathway analysis gives an indication of which biological or disease-relevant pathways the genes may be involved in [220]. The GO terms 'structural constituent of ribosome', 'RNA binding', 'endosome', 'mitochondrial envelope', 'mitochondrial membrane', 'cellular respiration', 'translation', and 'cellular biosynthetic process' emerged as significantly associated with good performance (false discovery rate < 10\%). KEGG pathway analysis gave very similar results, with 'ribosome' and 'oxidative phosphorylation' being the most highly enriched pathways (Fig. 3.1.5B). The GO terms associated inversely with good learning were 'glutamate receptor' and 'glutamate signaling pathway'. No KEGG pathways inversely associated with good learning were detected at the cutoff values chosen (false discovery rate $<10 \%$ ).

To estimate the possibility of false positives generated by the above procedure, the procedure was repeated twice with random permutations of the behavioral data. When the genes and correlation values derived from the permuted correlation analysis were analyzed by GSEA, a far lower number of GO terms or KEGG pathways emerged as statistically significant (Fig 3.1.5A).

\subsubsection{Over- and under-expressed pathways in high-performing aged mice}

After deriving a list of enriched pathways and biological functions in the high-performing animals, the individual gene expression levels of genes in the corresponding pathways were revisited.

The genes in the pathways ribosome/RNA-binding/translation, enriched in the animals who continued to perform well on the water maze task despite their age were studied further by comparing their expression levels in the 3 best- and worst-performing individuals.

Gene set enrichment analysis revealed a striking enrichment in genes in the GO category structural 'constituent of ribosome', and the KEGG pathway 'ribosome'. Both these gene sets contain ribosomal proteins that associate with rRNA to form the $40 \mathrm{~S}$ and $60 \mathrm{~S}$ eukaryotic ribosomal subunits. As seen from (Fig 3.1.6A and $\mathrm{B}$ ) a large proportion of the genes encoding ribosomal proteins in both subunits are expressed at higher levels in 'good learners' than in the 'poor learners'.

Gene expression levels of translation and ribosome-related genes in the top and bottom 3 animals are shown as part of the translation initiation pathway (Fig3.1.6B). This pathway was used for visualization to put the relationships between the components of the translational machinery into perspective. From Fig 3.1.6B it is clear that the best-performing animals have higher-than-average expression of several components of the translational machinery and that the opposite is true for the animals with poor performance.

GSEA also indicated that the high expression of glutamate receptors correlated with poor performance in old age. The average gene expression levels for the genes in the glutamate signaling pathway were calculated for the 3 animals with the best and worst performance on the water maze task. The ratio of these gene expression levels to mean expression of these genes in the entire group is represented in (Fig 3.1.6C). The 'poor learners' show high expression of several components of the pathways and the 'good 
learners' show low expression levels of these components.
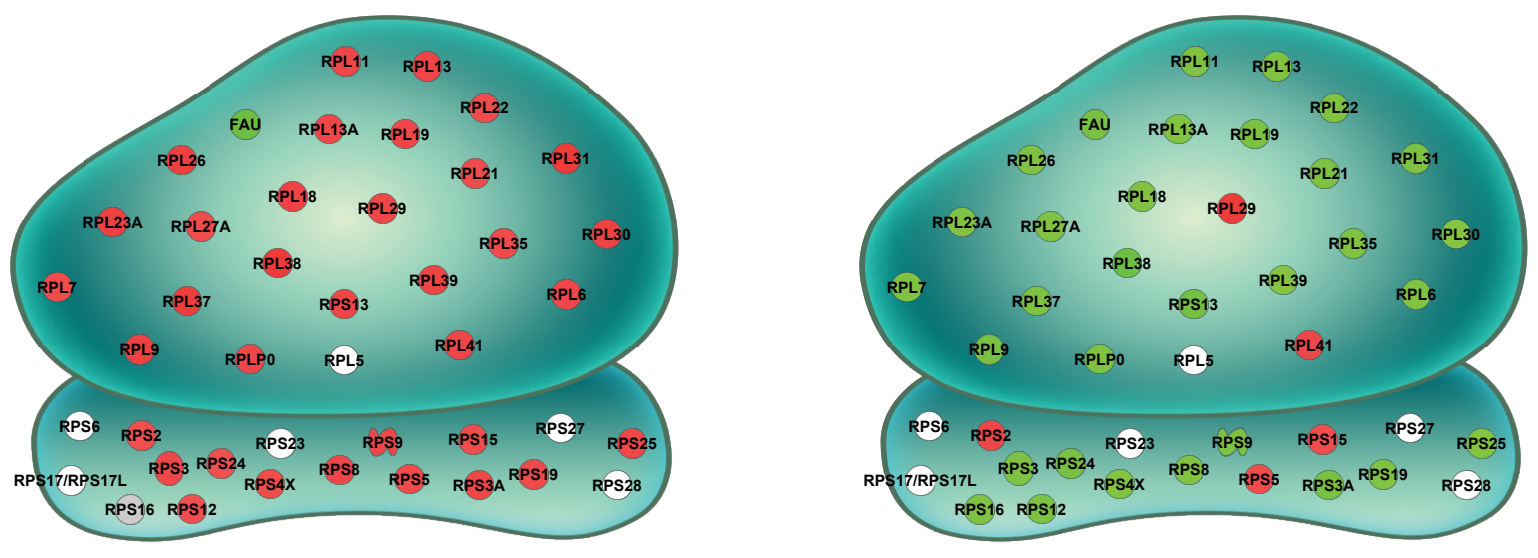

Figure 3.1.6.1 Diagram showing components of the murine ribosome

Average expression in the 3 highest performing mice (A) and the 3 lowest performing mice (B) Green represents expression below mean and red represents expression above mean. Color intensity depends on the ratio of expression levels of the gene to mean expression levels. Genes with prefix $L$ code for proteins in the large subunit and those with prefix $S$ code for proteins in the small subunit. Figure generated using Ingenuity IPA software. 


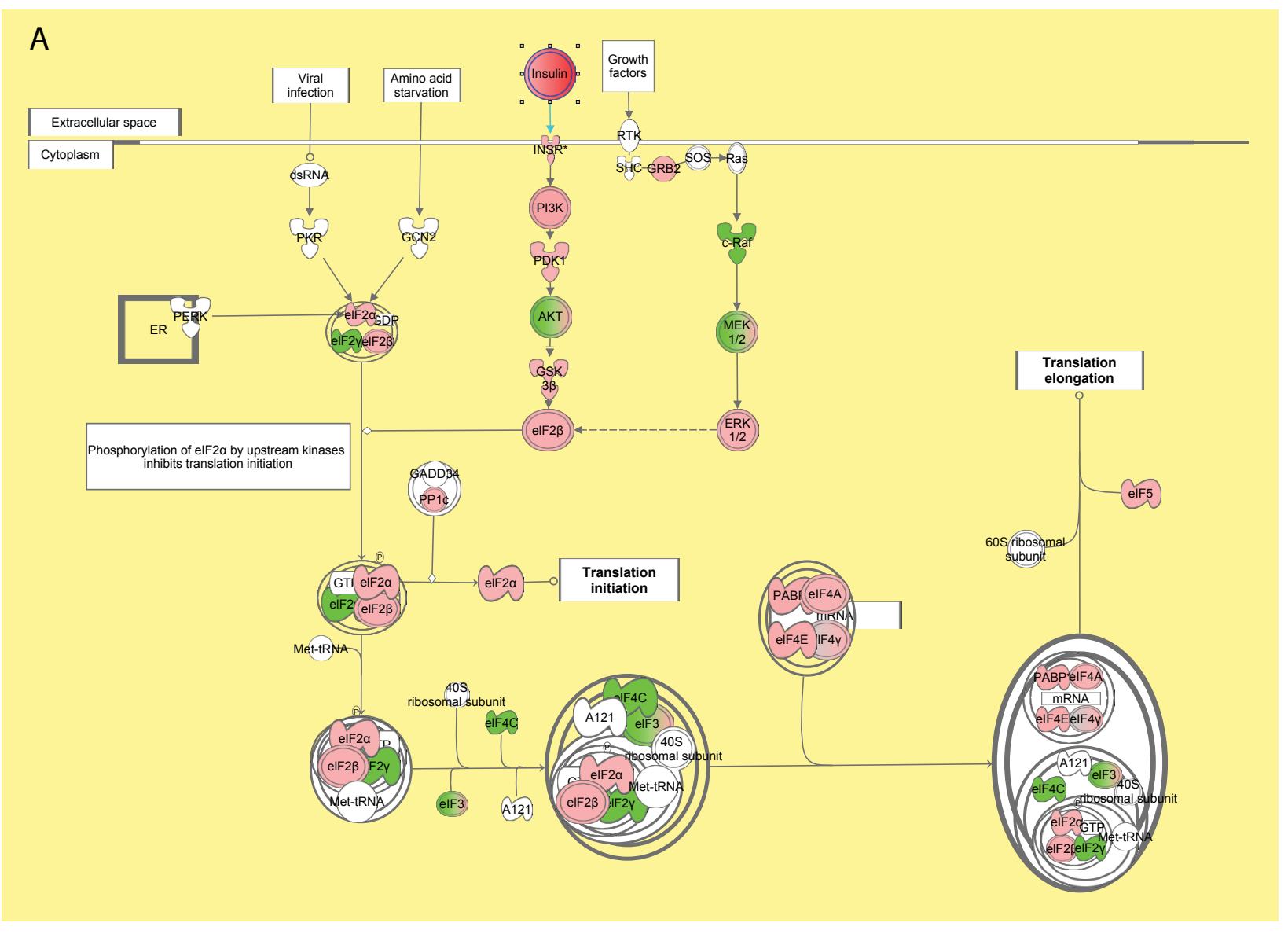




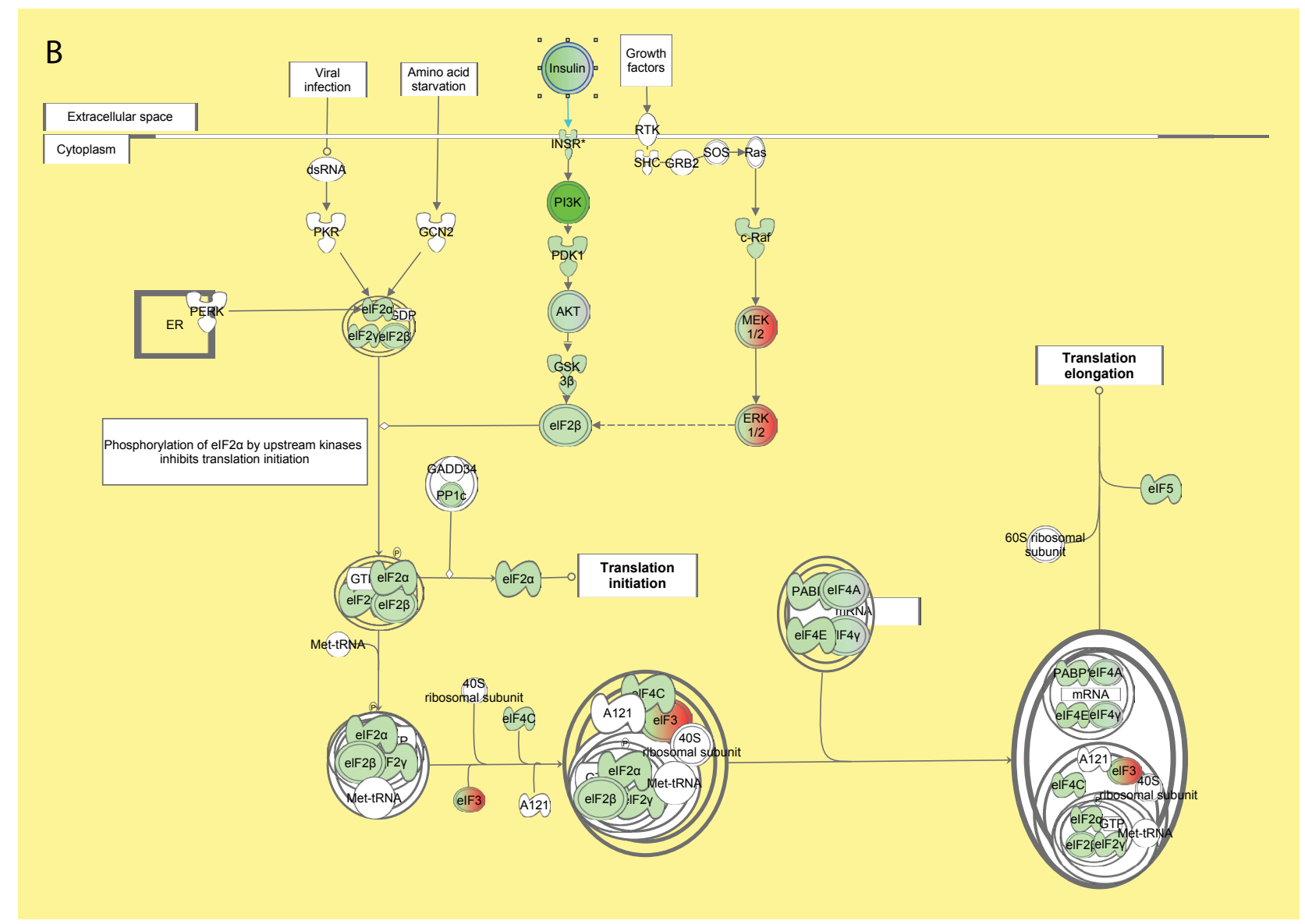

Figure 3.1.6.2 Translation initiation pathway diagram

Average expression in the 3 highest performing mice (A) and the 3 lowest performing mice (B) Green represents expression below mean and red represents expression above mean. Color intensity depends on the ratio of expression levels of the gene to mean expression levels. Symbols containing a mixture of green and red indicate that some isoforms of this protein were overexpressed and others underexpressed. Figure generated using Ingenuity IPA software. 


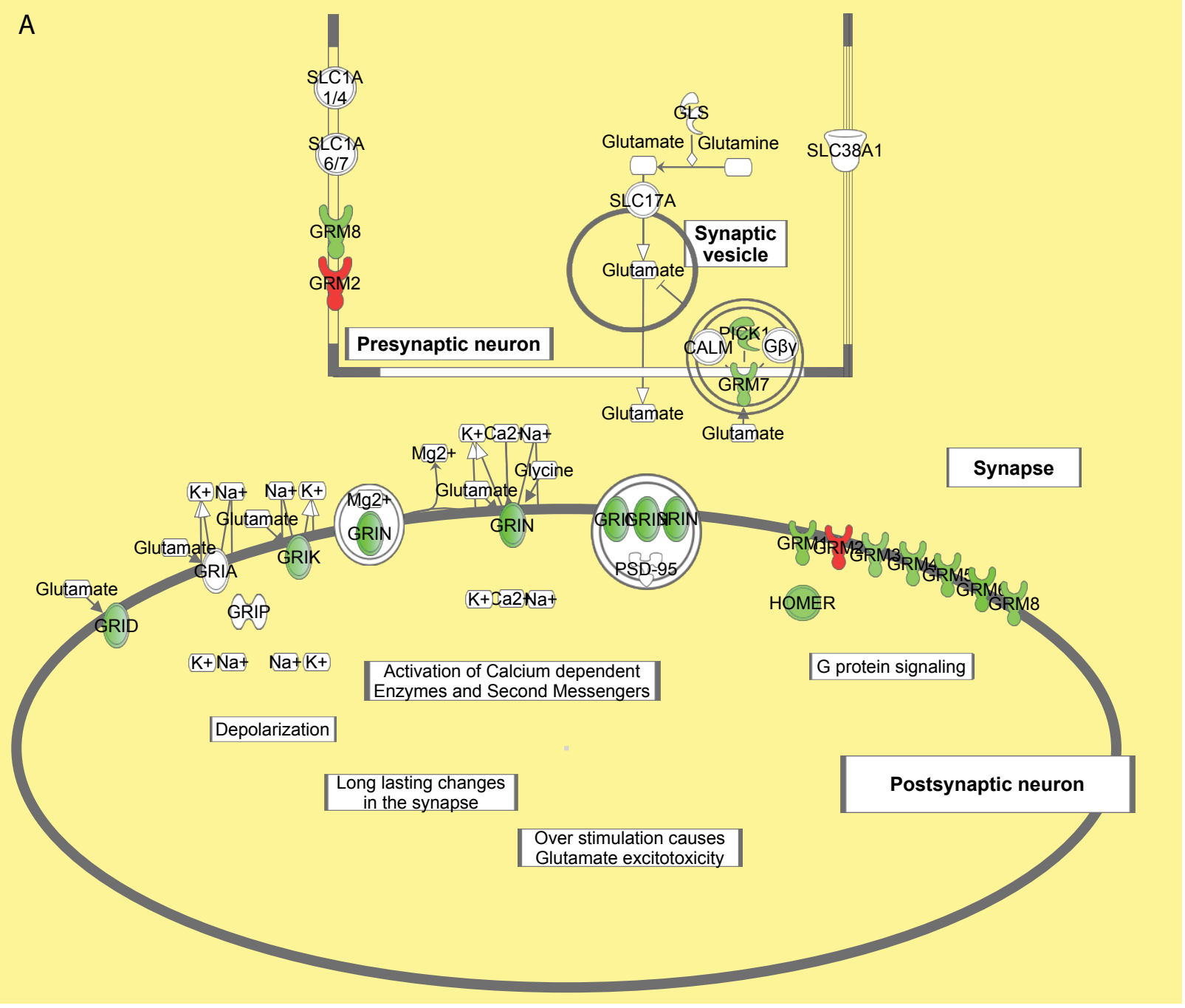




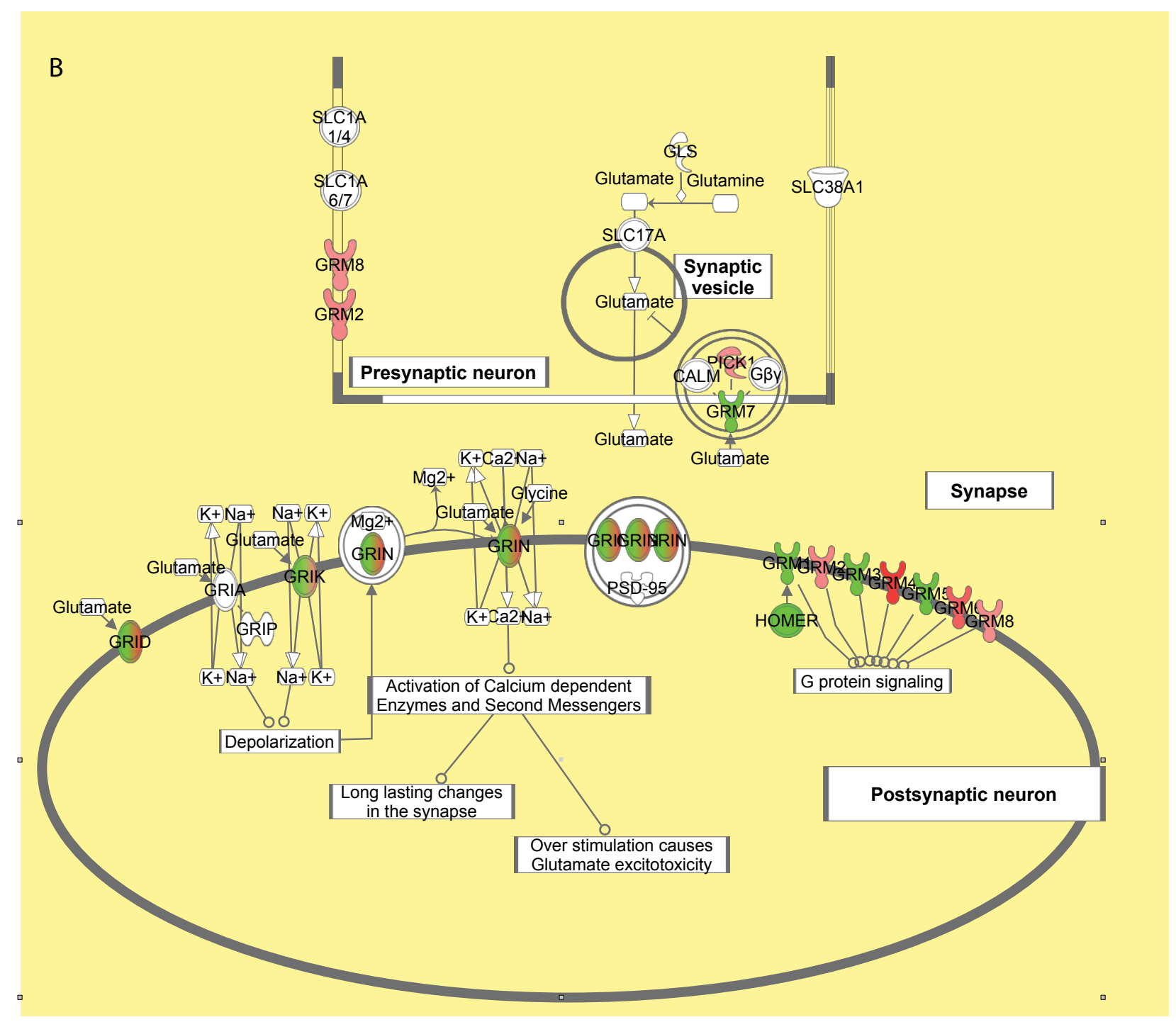

Figure 3.1.6.3 Glutamate signaling pathway diagram

A: Average glutamate receptor expression in the 3 highest performing mice B: Average glutamate receptor expression in the 3 worst performing mice. Green represents expression below mean and red represents expression above mean. Color intensity depends on the ratio of expression levels of the gene to mean expression levels. Symbols containing a mixture of green and red indicate that some isoforms of this protein were overexpressed and others underexpressed. Figure generated using Ingenuity IPA software. 


\subsubsection{MicroRNA expression in aging mice}

The expression of microRNAs in the same group of 16-month-old mice was analyzed in a manner similar to the mRNA. Pearson's correlations between the expression of each microRNA and the water maze probe test were calculated for every individual mouse. The expression levels of 16 microRNAs had a significant positive correlation with probe test performance and 11 had a significant negative correlation with probe test performance (Fig3.1.7 B and C).

To study the biological significance of these microRNA lists, the web-based 'Tool for analysis of microRNAs' (TAM) was used. TAM uses a relatively stringent algorithm to scan lists of microRNAs for over-representation of categories of biological such as families, clusters, biological pathways or using prior knowledge [221]. This method was developed as an alternative to the widely used alternative target-prediction based analyses of microRNA lists, which depend on sequence-motif based target prediction and result in a large number of false positives. Submitting the lists of microRNAs significantly correlated with probe test performance to the tool showed no significant enrichment for any family or cluster of microRNAs. 

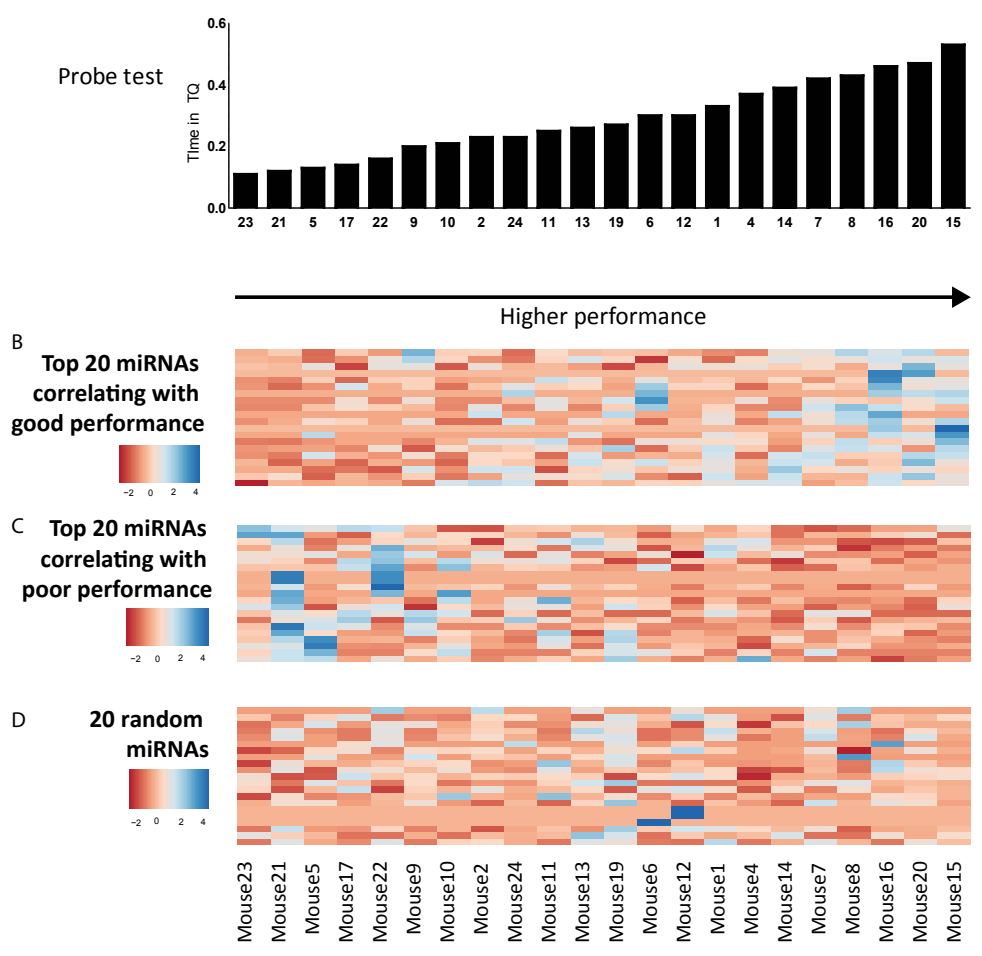
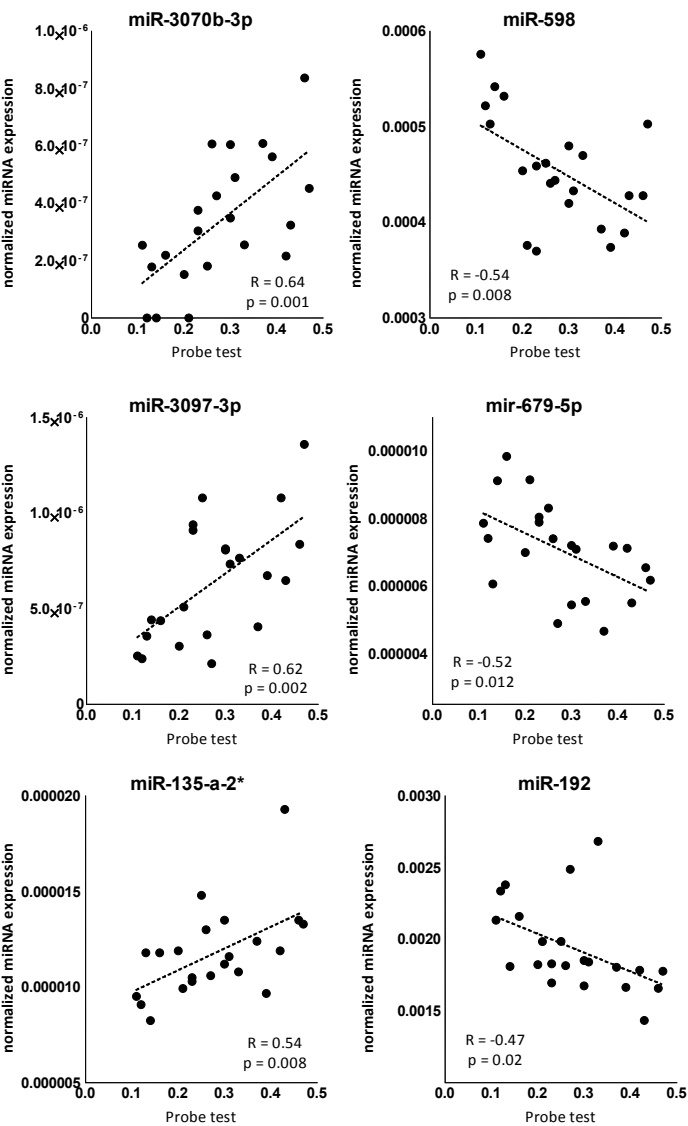

Figure 3.1.7 MicroRNA expression in aging mice

A: Time spent in target quadrant by 16 -month-old mice $(n=22)$, plotted individually and sorted from worst performace to best performance. Heat maps showing the scaled, normalized expression values of the 20 microRNAs most significantly correlated positively (B) and negatively (C) with performance on the probe test. Color scale represent levels of expression, with blue representing highest expression and red representing the lowest expression. D: Examples of correlations between performance and microRNA expression for the microRNAs mmu-miR-3070b-3p, -3097-3p, and -135a-2* which correlate positively with performance, and miR$598,-679-5 p$ and -192 whose expression correlates negatively with performance. The microRNA expression levels are plotted as normalied read counts on the vertical axis versus the probe test performance (percent time spent in target quadrant) on the horizontal axis. Deming regression lines are indicated and each solid circle represents an individual animal, $(n=22)$. 


\subsection{Alzheimer's disease}

\subsubsection{Gene and microRNA expression in the APP-PS1 mouse model}

To compare the mRNA and microRNA profile seen in age-associated memory impairment with that associated with amyloid pathology in AD, we used the double transgenic mouse strain APP-PS1. This strain was engineered to overexpress $2 \mathrm{AD}$-associated mutations in neurons and has been used widely as a mouse model for cerebral amyloidosis.

The APP-PS1 mice show neocortical amyloid deposition as early as 6-8 weeks of age. Hippocampal amyloid plaques are observed in the dentate gyrus at 2-3 months of age and in the CA1 at 4-5 months of age. The mice show no abnormalities in motor abilities or exploratory behavior but clear deficits in reversal learning and spatial memory are seen by 8 months of age $[222,223]$. However, like the other existing amyloid-based mouse models, the APP-PS1 mice show neither the tau pathology nor the frank neurodegeneration seen in human AD [222]. Nevertheless, they have been widely studied in the context of neurodegenerative disease and several aspects of their neuropathology associated have been reported. The APP-PS1 mice show decreased neurogenesis and disrupted functioning of hippocampal neuroprogenitor cells [224], decreases in learning-induced upregulation of gene expression [225], impaired LTP in the CA1 region, and impaired microglial motility and phagocytosis behavior. This strain has also been used to test therapies for amyloidassociated pathology such as HDAC inhibitors [223], microRNAs [226], plaque-targeted T cells [227], NMDA receptor antagonists [228], ApoE reduction [229, 230] and exposure to enriched environments [230].

Thus the molecular pathology and electrophysiology of these mice has been characterized, but little is known about the hippocampal transcriptomic response to overexpression of these human AD proteins.

Since the plaque pathology and cognitive deficits are well established by 8 months of age, we chose to compare that age to an earlier time point (4 months) to capture the early detrimental effects of the overexpression. The hippocampal transcriptomic profile in groups of APP-PS1 mice at both ages was studied by by RNA and small RNA sequencing and compared with that of their wild-type littermates. In the 4-month-old group 34 transcripts were differentially expressed in comparison to wild type mice, of which 22 were upregulated and 12 downregulated in the APPPS1 mice. The 8-month-old group showed more evidence of transcriptional changes, with 299 genes down regulated and 185 genes upregulated over controls.

The results of miRNA profiling were less informative - when an unsupervised clustering approach was used to study the intra- and inter-group similarity in microRNA expression, the transgenic mice did not form a distinct cluster (Fig 3.2.1B). After correcting $\mathrm{p}$ values for multiple testing, 16 out of the 855 expressed microRNAs showed a significant difference between groups. At 4 months of age, the microRNAs mmu-miR$3096 b,-409,-541,-221^{*}, 30 c-2^{*}$ and $-370^{*}$ were lower in the APP mice while mmu-miR-335, $-490,-700^{*}$, $-451,-142,-495,-136,-381^{*},-879^{*}$ and $-26 a-2^{*}$ were significantly raised in the APP mice (Fig 3.2.1C). At 8 months of age, the only microRNA differentially expressed between groups was mmu-miR-122. There were also no significant differences between microRNA expression in wild-types between 4 and 8 months of age. 
A

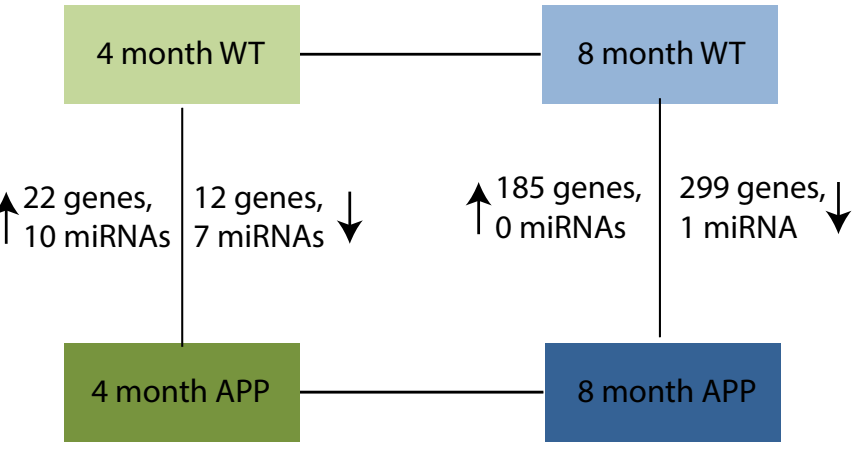

B
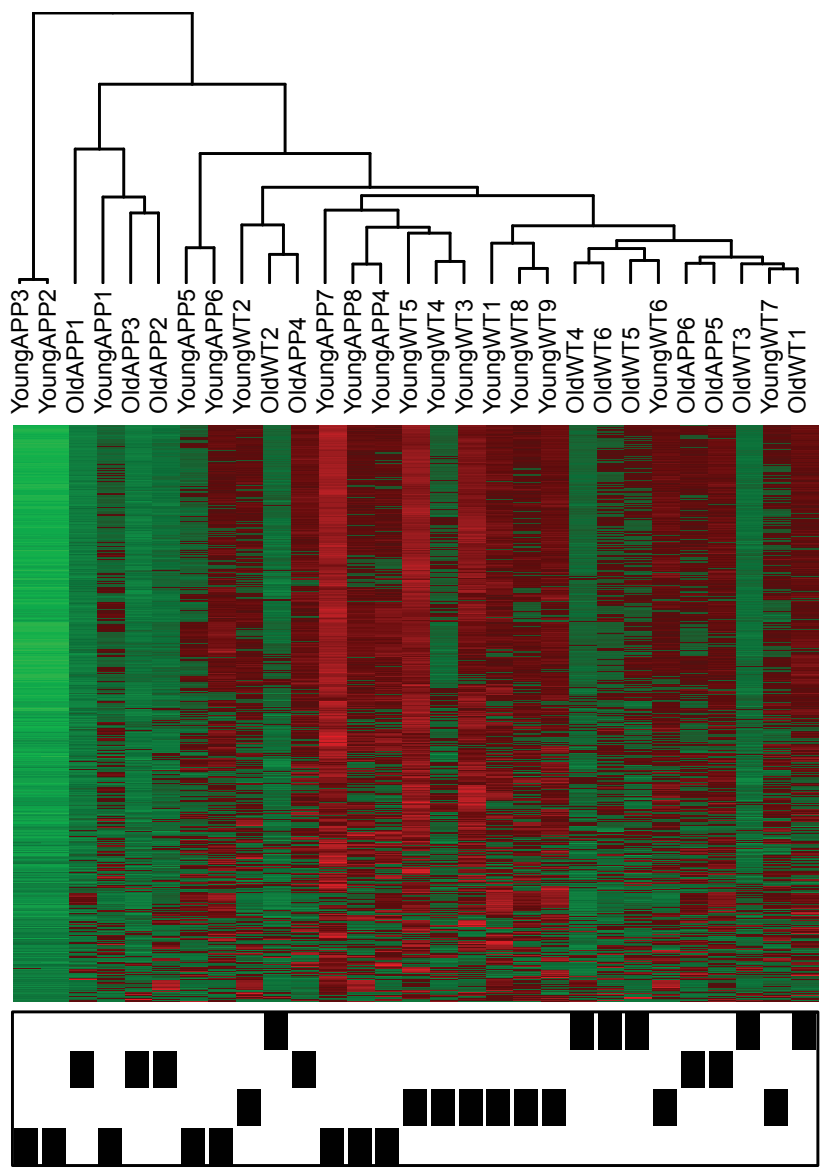

C 4 months downregulated

\begin{tabular}{|l|r|}
\hline id & FDR \\
\hline mmu-miR-136 & $1.70 \mathrm{E}-002$ \\
mmu-miR-879* & $1.70 \mathrm{E}-002$ \\
mmu-miR-142-5p & $1.70 \mathrm{E}-002$ \\
mmu-miR-335-3p & $1.70 \mathrm{E}-002$ \\
mmu-miR-26a-2* & $1.70 \mathrm{E}-002$ \\
mmu-miR-495 & $2.94 \mathrm{E}-002$ \\
mmu-miR-381* & $3.41 \mathrm{E}-002$ \\
mmu-miR-700* & $3.41 \mathrm{E}-002$ \\
mmu-miR-451 & $3.96 \mathrm{E}-002$ \\
mmu-miR-490-3p & $4.97 \mathrm{E}-002$ \\
\hline
\end{tabular}

4 months upregulated

\begin{tabular}{|l|r|}
\hline id & \multicolumn{1}{|l|}{ FDR } \\
\hline mmu-miR-370 & $2.03 \mathrm{E}-010$ \\
mmu-miR-541 & $5.56 \mathrm{E}-004$ \\
mmu-miR-30c-2* & $3.96 \mathrm{E}-003$ \\
mmu-miR-221* & $3.96 \mathrm{E}-003$ \\
mmu-miR-3096b-5p & $8.77 \mathrm{E}-003$ \\
mmu-miR-409-5p & $1.30 \mathrm{E}-002$ \\
\hline
\end{tabular}

8 months downregulated

\begin{tabular}{|l|l|}
\hline id & FDR \\
\hline mmu-miR-122 & \\
\hline
\end{tabular}

Figure 3.2.1 microRNA expression in the APP-PS1 mouse model

A: Scheme showing microRNA differential expression in APP and WT mice at the ages of 4 and 8 months. $B$ : Unsupervised clustering of samples by microRNA expression values. C: Differentially expressed microRNAs in APP mice at 4 months and 8 months of age (FDR = False discovery rate, FDR , 0.05) 


\subsubsection{RNA isolation from human cerebrospinal fluid}

Given recent reports that cells secrete RNA-containing vesicles into extracellular fluid under physiological conditions (see section 1.2.5), we tested various fractions of human cerebrospinal fluid for RNA (Fig 3.2.2A). CSF from two subjects was used to identify the fraction with the highest RNA yield. RNA was isolated from the large extracellular vesicle (EV), exosome, and exosome-free CSF fractions derived from $2 \mathrm{ml}$ of CSF from each patient and the size profile and the amount of RNA isolated was studied on a bioanalyzer. For both samples, the exosome fraction yielded the maximum total RNA (Fig 3.2.2B). The RNA yield differed between subjects, but the proportional distribution of RNA in the various fractions CSF remained constant.

Next-generation sequencing was carried out on the total RNA (derived from $2 \mathrm{ml}$ volume) from each fraction. As predicted by the bioanalyzer output, the exosome fraction had the most RNA reads. The exosomes also had the highest proportion of reads mapping to microRNA (Fig 3.2.2C). Further the number of microRNAs that could be detected, and the absolute number of microRNA reads was also highest in the exosome fraction (Fig 3.2.2D).

To confirm the results of next-generation sequencing qPCR for 10 miRNAs was performed on the exosome and exosome-free fractions. Of the 10 microRNAs tested, all were found in the exosome fraction, one was also found in the exosome-free CSF (Fig 3.2.2E). Notably, there were no microRNAs in exosome-free CSF that could not be detected in exosomes.

\section{Figure 3.2.2 RNA isolation from human cerebrospinal fluid (Facing page)}

A: Scheme showing CSF fractions and their isolation, B: Bioanalyzer picochip profiles, with size in nucleotides on the horizontal axis and quantity in fluorescent units (FU) on the vertical axis, of CSF exosomal RNA from a 71-year-old male subject without alzheimer's disease, C: Pie charts showing the RNA composition of each fraction, D: Graphs showing total RNA reads, total microRNA reads and number of microRNAs detected in each fraction. E: qPCR to detect 10 miRNAs in exosome and exosome-free fractions of CSF.

\begin{tabular}{|c|c|c|}
\hline E miRNA & Exosomes & Exosome-free CSF \\
\hline Hsa-miR-124a & + & + \\
Hsa-miR-181a & + & - \\
Hsa-miR-204 & + & - \\
Hsa-miR-21 & + & - \\
Hsa-miR-26a & + & - \\
Hsa-miR-29a & + & - \\
Hsa-miR-29b & + & - \\
Hsa-miR-29c & + & - \\
Hsa-miR-34a & + & - \\
Hsa-miR-34c & + & - \\
Hsa-miR-375 & + & - \\
\hline
\end{tabular}


A

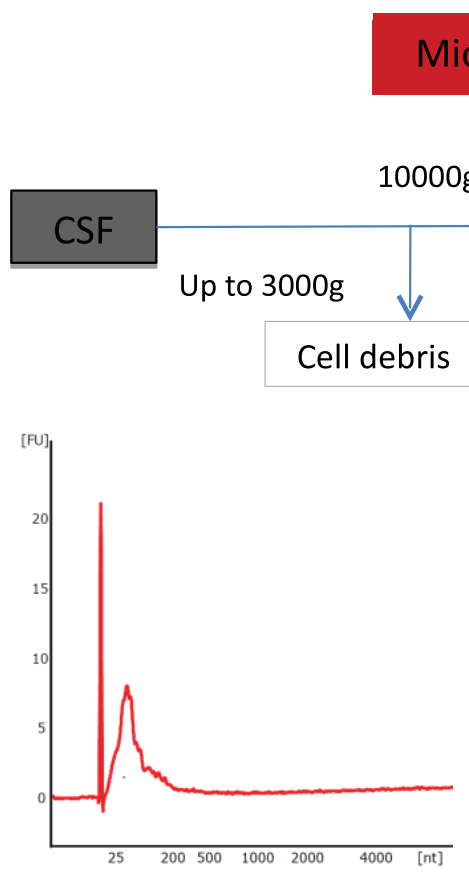

Microvesicles

C

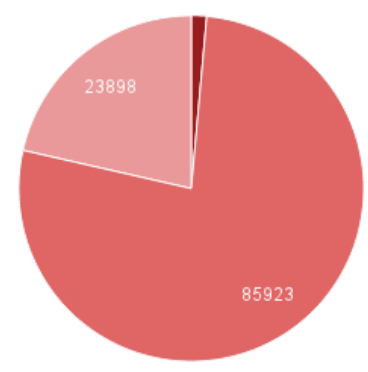

Microvesicles

D

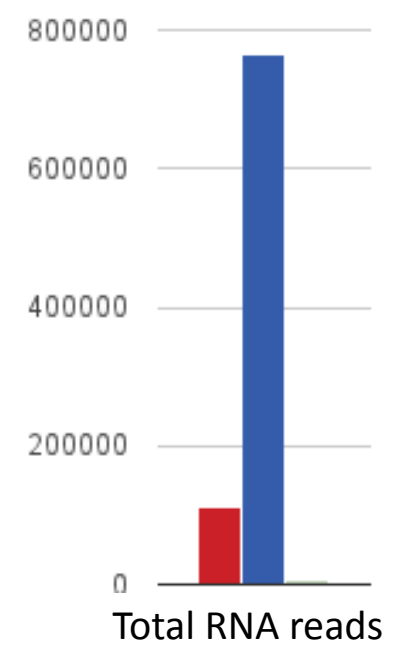

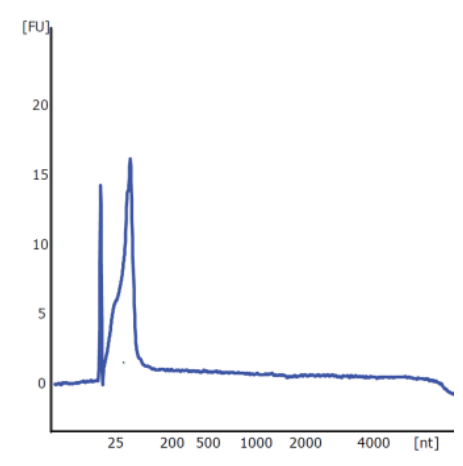

Exosomes
Exosomes $100000 \mathrm{~g}$

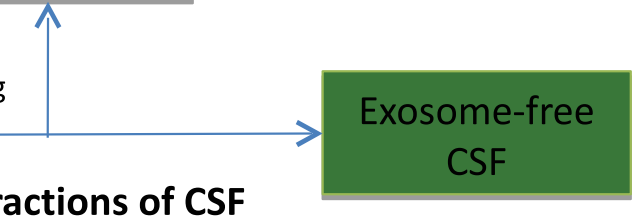

RNA-containing fractions of CSF

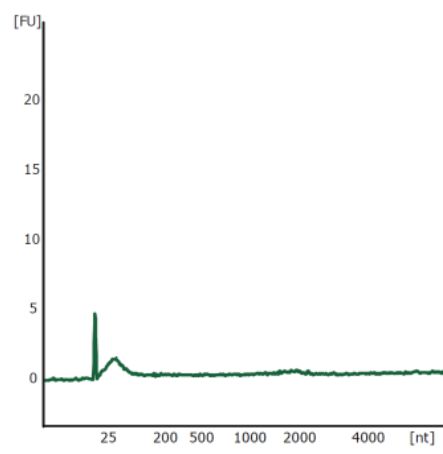

Exosome-free CSF

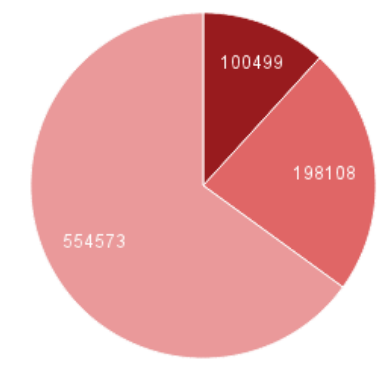

Exosomes

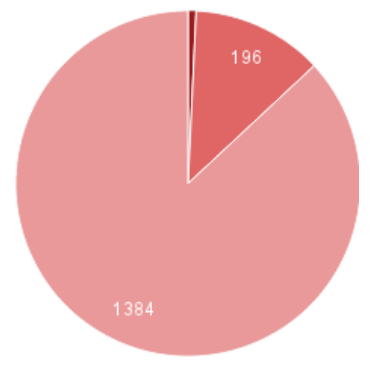

Exosome-free CSF
miRNA

Other small RNA

cDNA
200

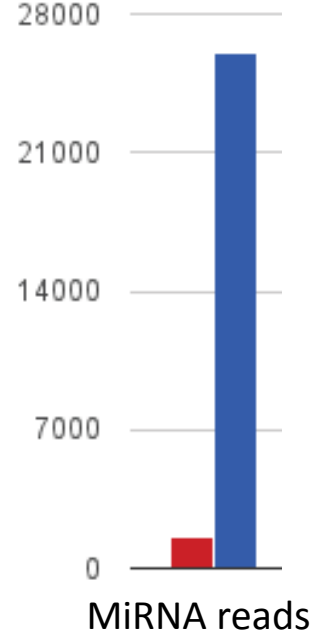

Microvesicles

Exosomes

Exosome-free CSF 


\subsubsection{The RNA contents of the CSF exosome}

For a better understanding of the exosomal RNA contents, we compared RNA from CSF exosomes to RNA from human plasma exosomes and from exosomes derived from primary hippocampal cell culture by NGS (Fig 3.2.3.1 and 3.2.3.2)

\subsubsection{Size profiles}

Comparing the size profile of CSF exosomal RNA to that of plasma exosomes and exosomes isolated from mouse primary cortical neuron cultures revealed that the profile was quite similar, with a peak in the small RNA region (20-200 nt), and very little evidence of larger RNA species (Fig 3.2.3.1A, B and C). In contrast, the size profile of cellular RNA from primary cortical neurons or mouse hippocampus (Fig 3.2.3.1 D and E) shows prominent large RNA peaks corresponding to the $18 \mathrm{~S}$ and $28 \mathrm{~S}$ ribosomal subunits.

To confirm that our exosomal RNA isolation procedure yielded pure RNA with no DNA contamination, we studied the bioanalyzer profile of RNA isolated from exosomes belonging to mouse primary cortical neuron cultures before and after treatment with RNase and DNase. As seen in (Fig 3.2.3.1 F-I) DNase treatment had no effect on the profile while RNase treatment completely digested the exosomal nucleic acid content.

\subsubsection{RNA contents of CSF exosomes}

To study the content of CSF exosomal RNA in further detail and to facilitate the identification of the various RNA species contained therein, we divided the RNA into 2 fractions and sequenced them separately. The size fractions corresponding to 0-40 nt (expected to be rich in miRNAs) (Fig 3.2.3.3) and 40-200 nt (containing the second peak) were sequenced (Fig 3.2.3.2) in RNA from 4 CSF exosome samples.

As expected, the 2 fractions were very different in their RNA contents (Fig 3.2.3.2B-E). The $0-40$ nt fraction contained about $9 \%$ miRNA, a small fraction of mRNA, and about half the reads mapped to other noncoding RNA. A large proportion of the reads from this fraction could not be mapped with confidence to the genome. By contrast, the 40-200 nt fraction consisted entirely of reads which could be mapped to noncoding RNAs other than miRNA.

The non-coding RNA in the exosomes is described below and the microRNA in Section 3.2.3.3.

The larger region (40-200nt) was found to contain about 2 million non-coding RNA reads, mapping to over 4500 non-coding RNAs. In comparison, the $2-40$ nt region contained about 200000 non-coding RNA reads (Fig 3.2.3.2 C). The large majority of the larger non-coding RNAs, including the most highly expressed ones have not yet been functionally characterized. 
A

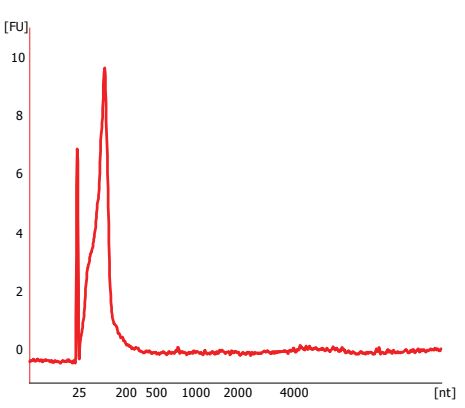

CSF exosomes

D

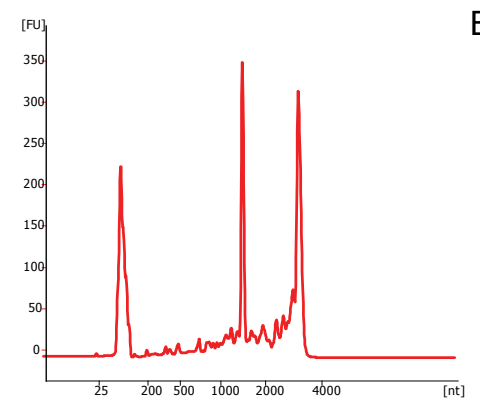

Mouse hippocampal tissue

E
B

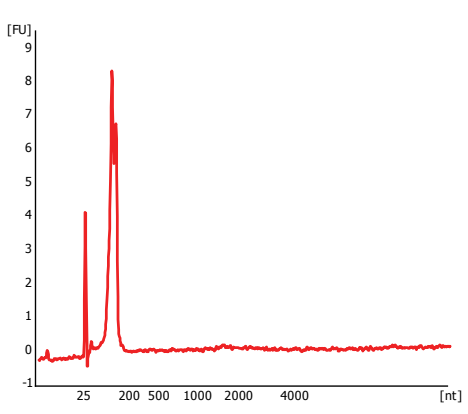

Plasma exosomes
C

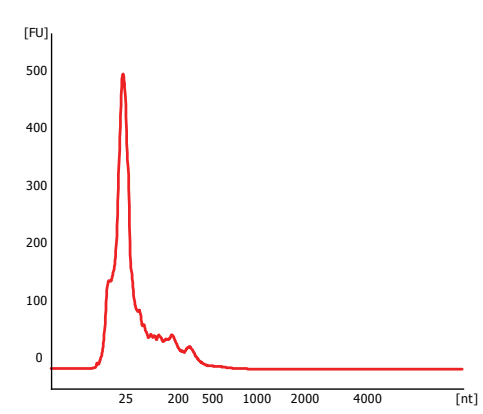

Primary neuron exosomes

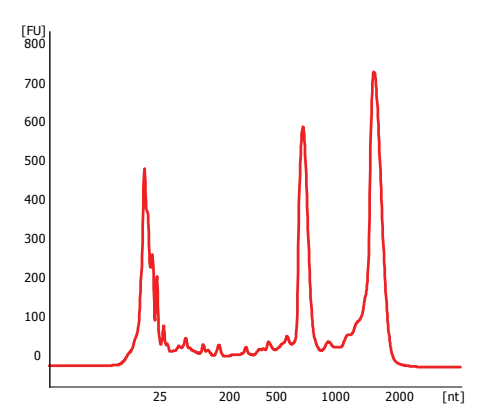

Primary hippocampal neurons

$\mathrm{F}$

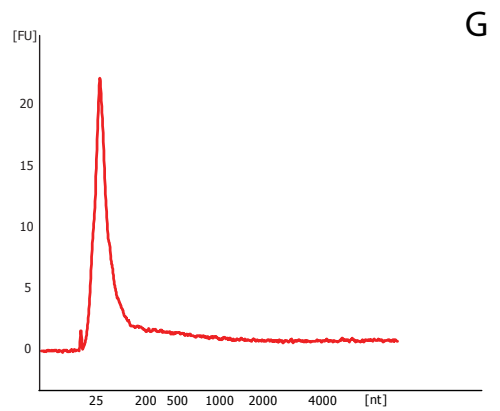

Untreated exosomal RNA
G

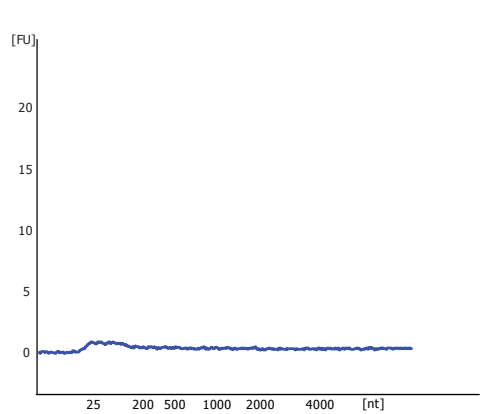

RNAse-treated

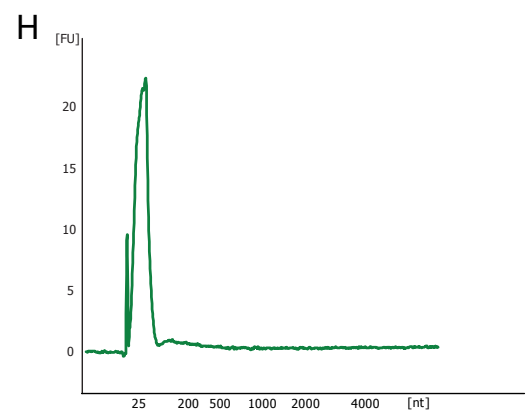

DNase-treated

\section{Figure 3.2.3.1 Exosomal RNA bioanalyzer profile}

Bioanalyzer RNA picochip profiles, with size in nucleotides (nt) on the horizontal axis and quantity in fluorescent units (FU) on the vertical axis A: RNA from human CSF exosomes, B: RNA from human plasma exosomes, C: RNA from exosomes in the culture medium from primary cortical neurons, D: RNA from primary cortical neurons in culture, E: RNA from whole mouse hippocampal tissue F: Untreated exosomal RNA, G: Exosomal RNA treated with DNase, H: Exosomal RNA treated with RNase and I: The first 3 profiles overlaid. 
A

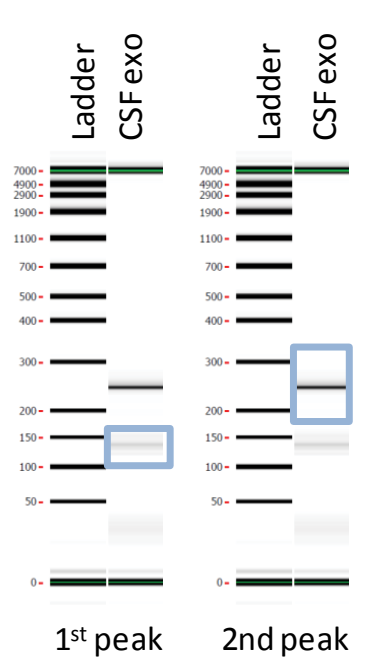

D Fraction1(20-40nt)

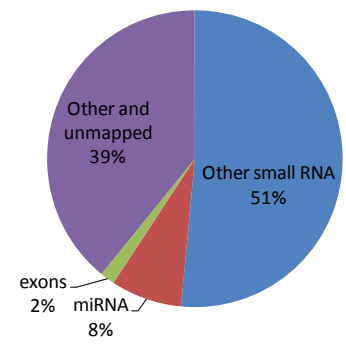

$\mathrm{E}$

Fraction1(20-40nt)

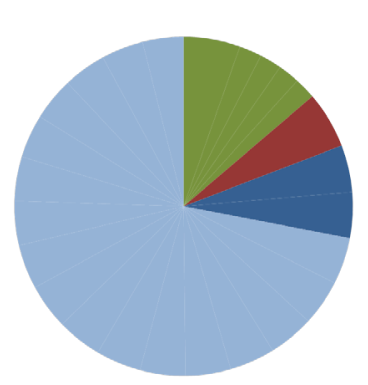

B

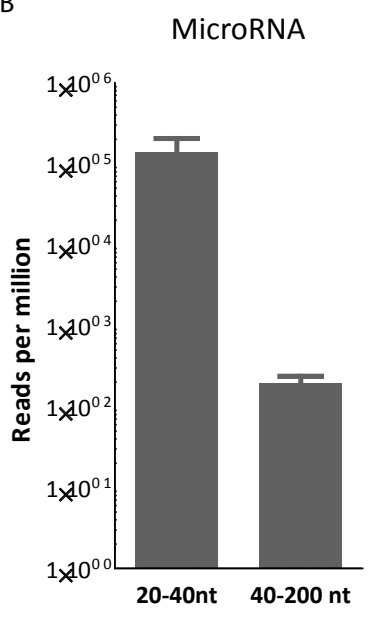

C

OTher non-coding RNA

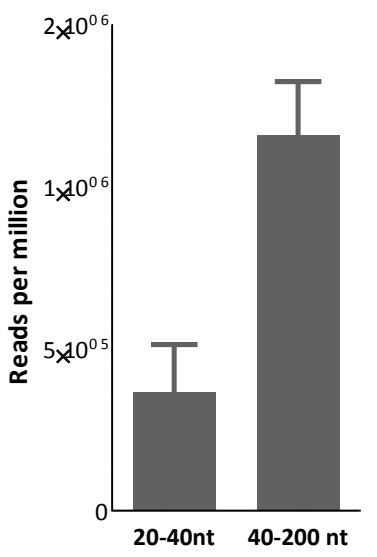

Fraction2(40-400nt)

exons

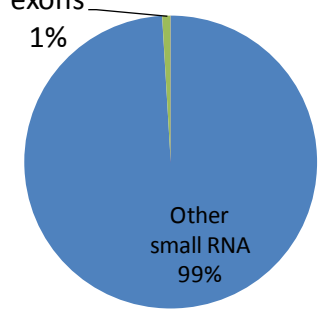

Fraction2(40-400nt)

\begin{tabular}{|c|c|}
\hline NEAT1 & - RP11-982M15.8 \\
\hline - RP11-81H14.2 & - RP11-63M22.1 \\
\hline - RP11-488P3.1 & - RP11-161M6.2 \\
\hline RP11-1C8.5 & — RP11-685M7.3 \\
\hline LINC00649 & = BX284613.1 \\
\hline$=\mathrm{RP} 4-749 \mathrm{H} 3.1$ & = RP4-791K14.2 \\
\hline = AL357519.2 & = AL158077.1 \\
\hline AC002066.2 & = AC048346.1 \\
\hline RP5-1120P11.1 & $\approx \mathrm{AC} 116038.1$ \\
\hline AC034154.1 & $\square \mathrm{AC012003.1}$ \\
\hline LA16c-306A4.2 & $\approx \mathrm{AC} 012305.1$ \\
\hline$=\mathrm{AL} 391538.1$ & $\square \mathrm{AC} 109357.1$ \\
\hline RP3-522J7.6 & \\
\hline
\end{tabular}

Figure 3.2.3.2 Exosomal RNA content

A: Average number of reads per million that map to microRNA $(n=4)$, B: Average number of reads per million that map to microRNA $(n=4)$, C: Average number of reads per million that map to other (nonmiRNA) non-coding RNA ( $n=4$, logarithmic scale), D: RNA distribution within fraction 1(20-40nt) and fraction 2(40-200nt) of CSF exosomal RNA, E: 25 most highly expressed non-coding RNAs from NGS of fraction 1(20-40nt) and fraction2 (40-200nt)

\section{Figure 3.2.3.3 Exosomal microRNA content (Facing page)}

A: Approximate size of the RNA fraction used for sequencing (0-40nt) B: Pie charts representing the relative proportion of microRNA, small non-coding RNA and other coding or unknown RNA in CSF exosomes, plasma exosomes and exosomes from primary hippocampal cultured neurons. Both CSF and plasma exosomes were derived from the same patient, a 64-year-old male with Alzheimer's disease. C: Average number of microRNAs detected in CSF exosomes, plasma exosomes and exosomes from primary hippocampal cultured neurons. between samples, with one sample on the horizontal axis 
A
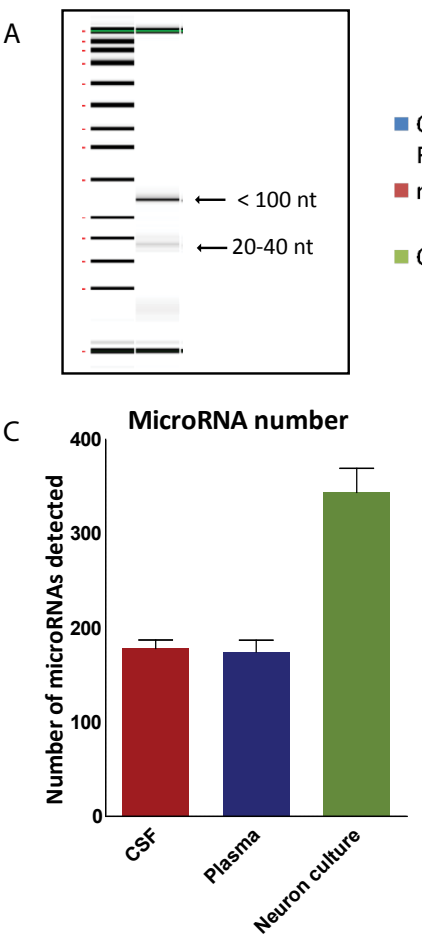

E

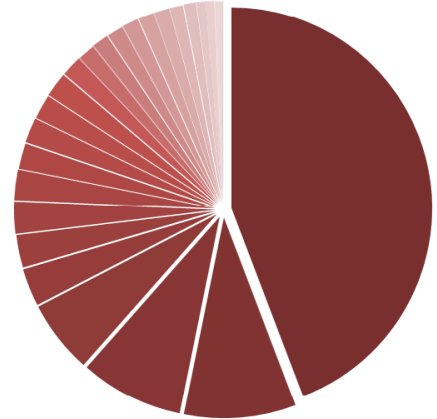

CSF exosomes

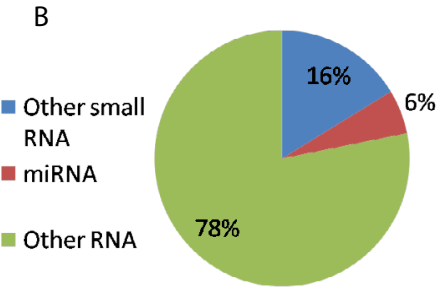

CSF exosomes

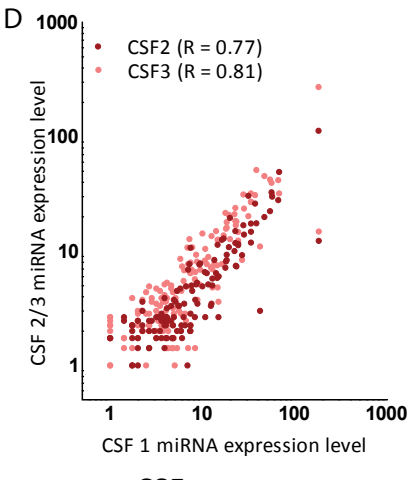

CSF exosomes

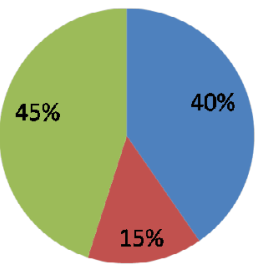

Plasma exosomes

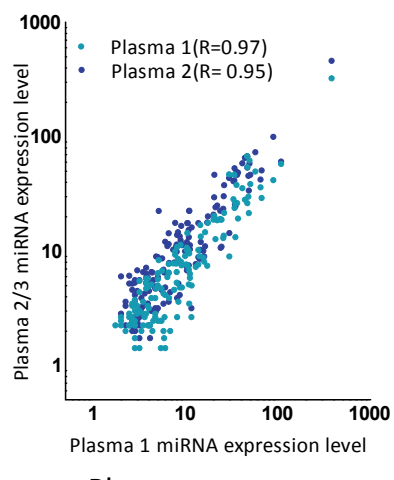

Plasma exosomes

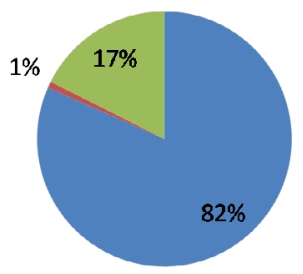

Primary neuron exosomes

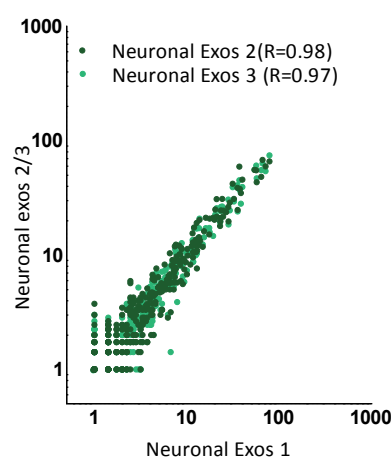

Primary neuron exosomes

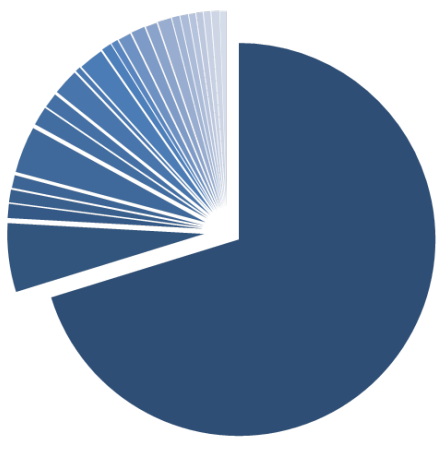

Plasma exosomes

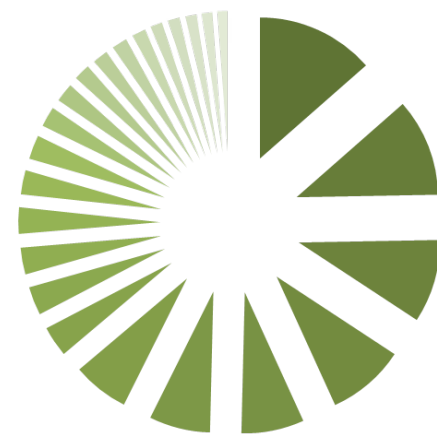

Primary neuron exosomes

\begin{tabular}{|c|c|}
\hline a hsa-miR-204-5p & " hsa-miR-99b-5p \\
\hline hsa-miR-92b-3p & hsa-let-7e-5p \\
\hline a hsa-miR-10b-5p & hsa-miR-30a-5p \\
\hline hsa-miR-486-5p & hsa-miR-100-5p \\
\hline hsa-let-7f-5p & hsa-miR-22-3p \\
\hline hsa-miR-92a-3p & hsa-miR-151a-3p \\
\hline hsa-miR-191-5p & hsa-miR-127-3p \\
\hline hsa-miR-28-3p & hsa-miR-182-5p \\
\hline Wsa-miR-181a-5p & hsa-miR-26a-5p \\
\hline Wsa-miR-148a-3p & hsa-miR-9-5p \\
\hline hsa-miR-10a-5p & hsa-let-7c \\
\hline hsa-miR-143-3p & hsa-miR-192-5p \\
\hline
\end{tabular}

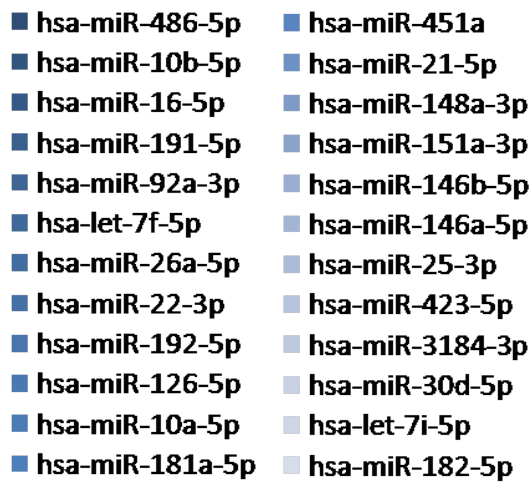

\begin{tabular}{ll}
$\square$ mmu-miR-181a & mmu-let-7i \\
$\square$ mmu-miR-22 & mmu-miR-16 \\
mmu-let-7c & mmu-miR-191 \\
\hline mmu-miR-2182 & mmu-miR-100 \\
\hline mmu-miR-26a & mmu-miR-92a \\
\hline mmu-miR-9 & mmu-miR-30a \\
\hline mmu-let-7f & mmu-miR-181b \\
\hline mmu-miR-99b & mmu-miR-30d \\
\hline mmu-miR-125b-5pmmu-miR-30e \\
mmu-let-7a & mmu-let-7b \\
mmu-miR-127 & mmu-miR-151-3p \\
mmu-miR-92b & mmu-miR-148a
\end{tabular}

Fig 3.2.3.3 D. Scatterplots showing correlations in miRNA expression versus 2 other randomly selected samples on the vertical axis. Each dot represents an individual miRNA and R stands for Pearson's correlation coefficient. For exosomes from primary hippocampal cell, the 3 exosome samples were collected from independent culture dishes. Plasma and CSF are from 3 different subjects. E: The top 30 microRNAs in CSF exosomes, plasma exosomes and exosomes from primary hippocampal cultured neurons. 


\subsubsection{Small RNA and microRNA}

The majority of small RNA is the CSF exosomes is non-microRNA small non-coding RNA. A large number of transcripts also derive from coding regions. MicroRNA forms not more than $15 \%$ of total exosomal RNA in exosomes from either CSF, plasma or primary neuronal culture (Fig 3.2.3.3B). Despite the relatively low overall proportion of microRNAs in exosomal RNA, the number of microRNAs that can be detected is large. We were able to detect at least 150-250 microRNAs in each sample of CSF exosomes and 100-200 in each sample of plasma exosomes. In total, 412 different microRNAs could be reliably detected in CSF exosomes across all samples (> 10 reads). In exosomes from primary hippocampal neurons, although the proportion of microRNA appears to be even lower, about 500 microRNAs could be detected (Fig3.2.3.3C). The microRNA profile obtained by NGS of exosomes appears to be highly technically reproducible. After applying a square-root transform to control for undue influence by highly expressed microRNAs, correlations in microRNA expression levels between samples were measured. In exosomes from primary hippocampal cultures, the inter-sample correlations were above $90 \%$. The correlations in CSF and plasma were from $70-80 \%$. Considering that these comparisons were performed in unrelated individuals with varying health status, including both patients with $A D$ and healthy controls, these results indicate a high degree of general similarity in the exosomal RNA expression patterns. The microRNA expression pattern, however, differs widely depending on the biological fluid used as a source of exosomes. There are no obvious similarities in microRNA expression levels between exosomes from CSF, plasma and primary neurons (Fig 3.2.3.3E).

\subsubsection{Exosomal RNA versus cellular RNA}

After verifying that the small amounts of RNA isolated from CSF exosomes can be studied by NGS, the next step was to study the relationship between exosomal RNA and RNA in the cell that secretes them. To enable a direct comparison RNA was isolated from primary hippocampal neurons in cell culture at 14 days in vitro and from the exosomes isolated from the growth medium of these cells. As shown in Fig 3.2.3.1, exosomal RNA is almost exclusively in the 20-200nt size range, while cellular RNA contains larger species, with the $18 \mathrm{~S}$ and $28 \mathrm{~S}$ subunits of ribosomal RNA forming prominent peaks. NGS of exosomal and cellular small RNA revealed that certain microRNAs appear to be selectively exported into exosomes, and others selectively depleted (up to 35 -fold in both directions) (Fig 3.2.3.4A). Further, not all cellular microRNAs can be detected in exosomes, and not all exosomal microRNAs can be detected in cells (Fig 3.2.3.4B).

To determine if exosomal RNA changes after perturbation of the cells in vitro, exosomes isolated healthy cultures (control) were compared with those isolated from cultures treated with amyloid-beta (Fig 3.2.3.4D). NGS revealed that cells and exosomes both contain a relatively small proportion of microRNA, and exosomes are not enriched in microRNA compared to cells, but rather contain a slightly lower proportion of miRNA than the corresponding size fraction in the cell. After treatment with A-beta, a shift in distribution of RNA was observed. In primary neurons, there was an increase in the proportion of other small non-coding RNA (non-miRNA) with a corresponding decrease in mRNA and long-non-coding RNA. These changes were reversed in exosomes, with a remarkable increase in proportion of reads mapped to mRNA and longnon-coding RNA and a decrease in small non-coding RNA. The proportion of microRNA remained relatively unchanged after treatment with A-beta. 
A-beta treatment causes changes in microRNA expression pattern of both cells and exosomes, but exosomal changes do not parallel cellular changes. There is little overlap between the microRNAs differentially expressed after A-beta treatment in primary neurons and exosomes isolated from those neurons. However the changes in both cells and exosomes are clear enough to be detected by an unsupervised clustering algorithm. Exosomes from A-beta-treated cells and exosomes from untreated cells form distinct clusters (Fig 3.2.3.4 F).

A

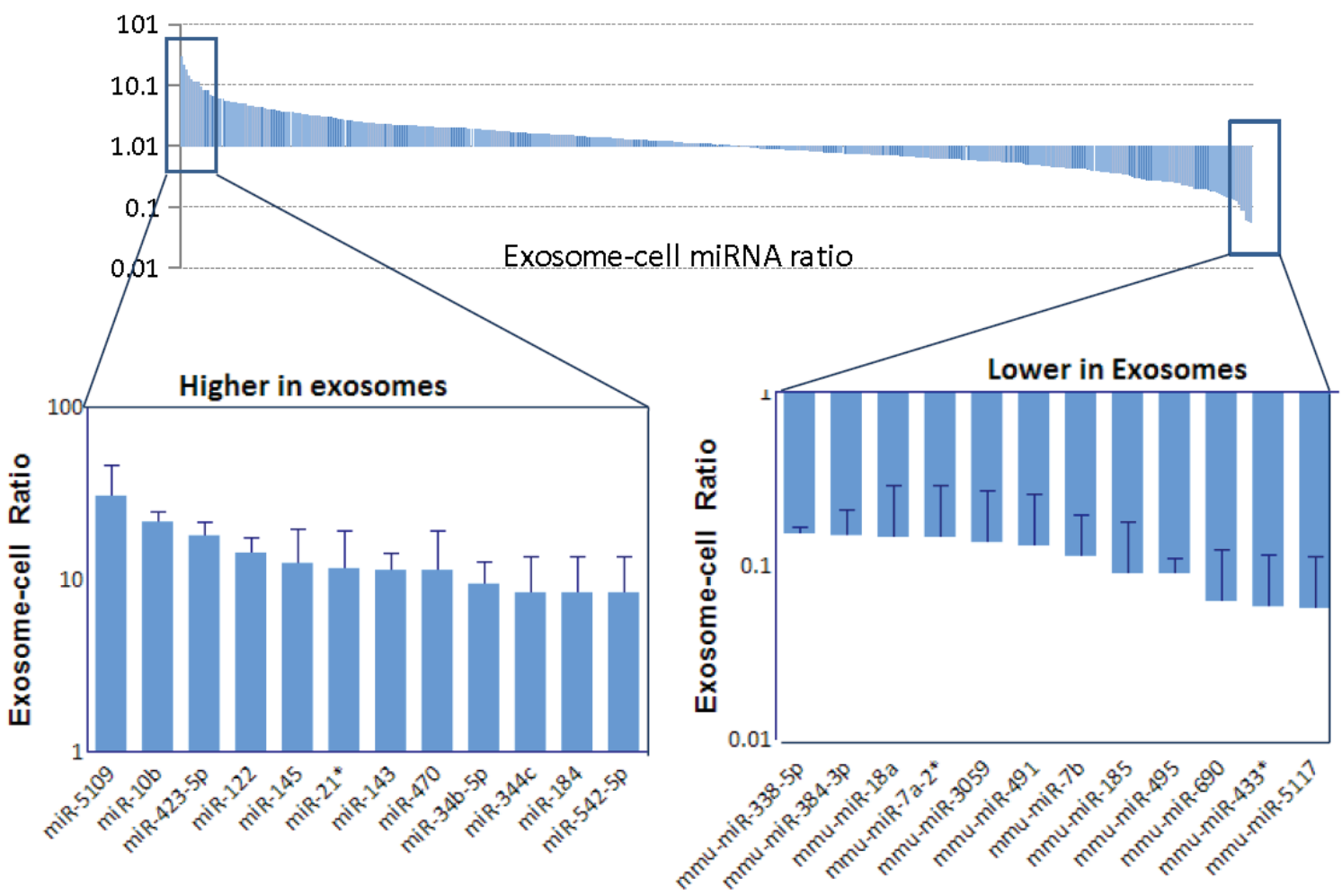

B

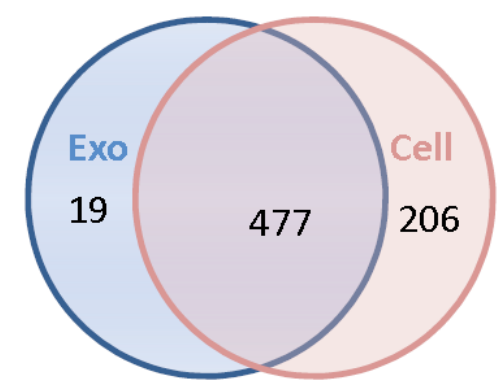

Figure 3.2.4 Exosomal RNA versus cellular RNA

A: Average ratio of exosomal level to cellular level for all microRNAs expressed in both exosomes and cells and miRNAs with the highest and lowest exosome-to-cell expression ratio $(n=3$ per group, error bars represent SEM) B: Venn diagram showing microRNAs detectable in cells and exosomes and the overlap between them 


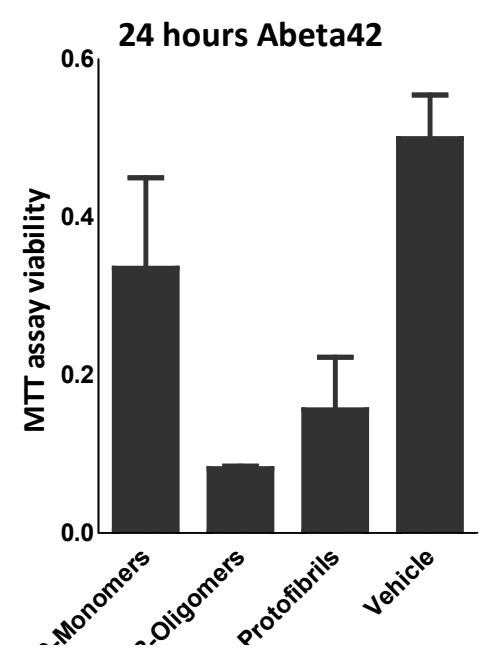

\section{Ctrl Cells \\ Ctrl Exosomes}

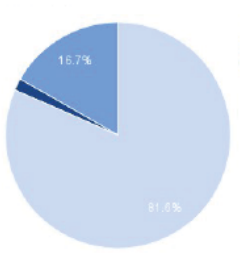

E

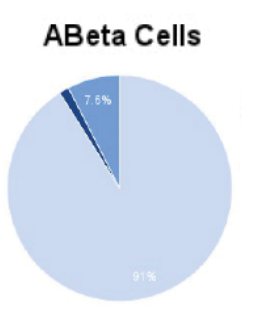

ABeta Exosomes

$\mathrm{F}$

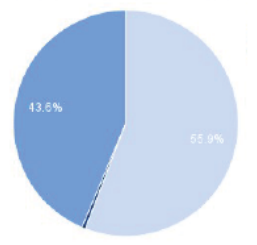

Other small RNA

miRNA

mRNA and other non-small RNA

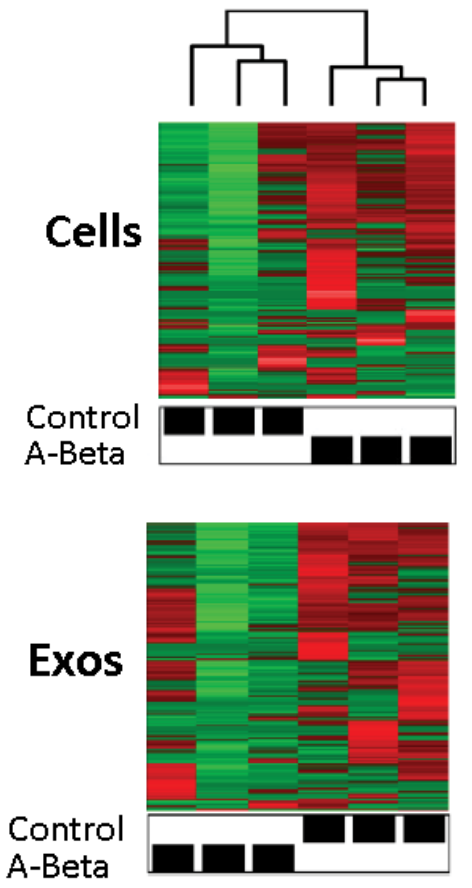

\begin{tabular}{|l|c|}
\hline \multicolumn{1}{|c|}{ id } & P Val \\
\hline mmu-miR-34c & 0.0000921 \\
mmu-miR-153 & 0.0028902 \\
mmu-miR-29b & 0.0034034 \\
mmu-miR-433* & 0.0113655 \\
mmu-miR-1839-5p & 0.0120891 \\
mmu-miR-322 & 0.0156360 \\
mmu-miR-344d & 0.0246580 \\
mmu-miR-501-3p & 0.0269427 \\
mmu-miR-222 & 0.0278270 \\
mmu-miR-872 & 0.0291604 \\
mmu-miR-34a & 0.0309230 \\
mmu-miR-1198-5p & 0.0311310 \\
mmu-miR-690 & 0.0319231 \\
mmu-miR-377 & 0.0414229 \\
mmu-miR-9* & 0.0448850 \\
mmu-miR-135a & 0.0489870 \\
\hline
\end{tabular}

\section{Figure 3.2.4 Exosomal RNA versus cellular RNA}

C: MTT assay to assess viability of treated neurons after treatment with A-beta 42 oligomers, vehicle control (control1) and A-beta 40 monomers (control2) D: Pie charts showing proportion of reads mapping to RNA categories (single representative images, original experiment was performed in 3 replicates). E: microRNAs differentially expressed in neurons and exosomes after A-beta treatment $(n=3$ per group, unadjusted $p$ value $<0.05) \mathrm{F}$ : Unsupervised clustering of A-beta treated exosomes and cells, based on all expresed microRNAs 


\subsubsection{Sequencing RNA from small volumes of CSF}

If RNA isolated from cerebrospinal fluid exosomes is to be used for studying brain disease on a larger scale, the procedure must be feasible from small amounts of starting material. CSF stored in biobanks for clinical studies is typically available in volumes of $1-2 \mathrm{ml}$. Using the RNA isolation protocol described above, the amount of exosomal RNA isolated from $1 \mathrm{ml}$ of CSF was on the order 3-4 nanograms, with considerable variation from sample to sample (Fig 3.2.3.5A). In order to ensure that RNA sequencing could be reproducibly performed from these small starting amounts, we performed serial dilutions from a pool of CSF exosomal RNA, sequenced them and compared the results. As seen in Fig 3.2.3.5B, the sequencing protocol is highly reproducible for starting amounts as low as $0.5 \mathrm{ng}$ of RNA. Further, these small starting volumes were used to check if CSF samples from 2 different subjects could be separated. Interestingly, as little as $0.25 \mathrm{ng}$ of RNA from CSF exosomes was enough to distinguish between 2 subjects by an unsupervised clustering algorithm (Fig 3.2.3.5C).

\subsubsection{CSF RNA in Alzheimer's disease}

The final step was to compare CSF exosomal RNA between AD patients $(n=18)$ and control subjects $(n=20)$ by next generation sequencing.

\subsubsection{Differential expression and unsupervised clustering}

After studying the CSF exosomal RNA content by next generation sequencing, the next step was to test if this exosomal RNA could be of use in further understanding the pathophysiology of AD. CSF exosomal RNA from 18 AD patients and 20 controls was sequenced and differential expression of microRNAs was analyzed (Fig 3.2.6.1B). After adjusting the $\mathrm{p}$ value by the Benjamini-Hochberg procedure for multiple testing, a list of 7 microRNAs (hsa-miR-192-5p, -486-5p, -598, -122-5p, -25-3p, -375, and -3591-3p ) were significantly lower in the CSF exosomes of patients with AD. Of a total of 412 expressed miRNAs, 41 microRNAs were found to be differentially expressed with a significance level of less than 0.05 ( $p$ value unadjusted for multiple testing) 12 were higher in $A D$ patients than in controls and 19 were lower.

The microRNA expression was then used to classify the samples using an unsupervised clustering algorithm. The samples clustered into 2 large groups, with one cluster having predominantly AD patients and the other cluster predominantly control subjects, but perfect separation could not be achieved (Fig 3.2.6.1 A). Notably, several other potential confounding factors such as age, gender or medication intake also did not seem to have an effect on sample clustering, but the medical facility of origin of the samples (center where the CSF was withdrawn and stored before exosome isolation) did appear to influence the detected RNA expression profile (Fig 3.2.6.1 A). 
A

Average RNA in CSF exosomes CSF exosomal RNA serial dilutions

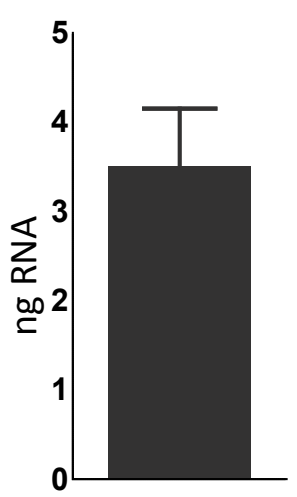

$2 \mathrm{ml} \mathrm{CSF}$
B

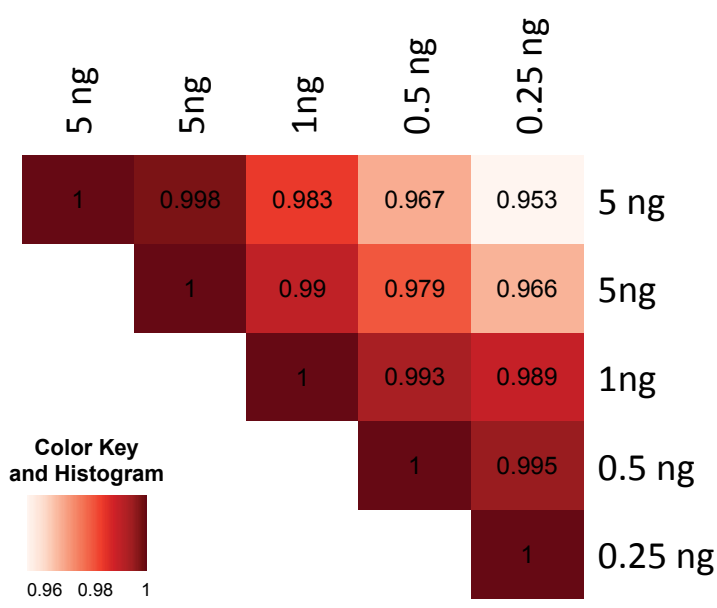

C

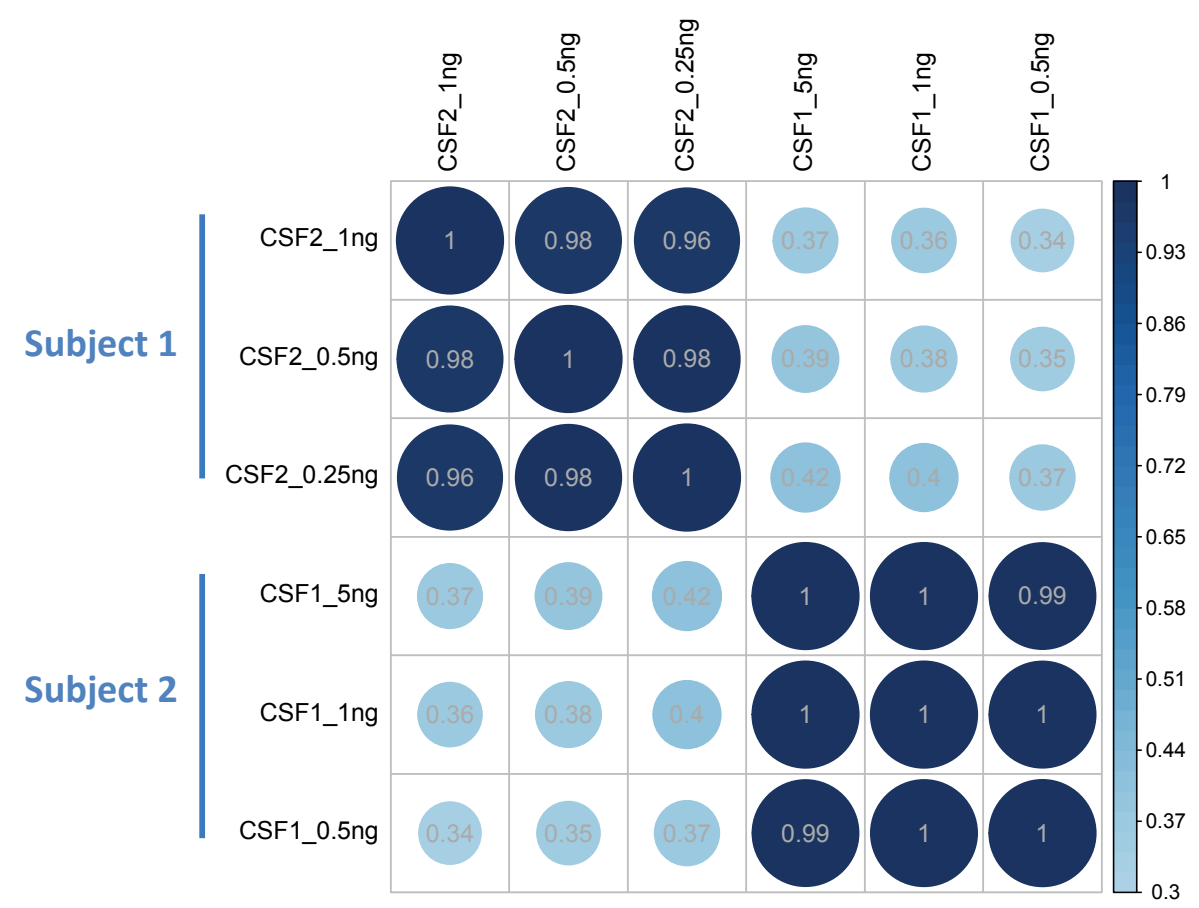

\section{Figure 3.2.5 Sequencing RNA from small volumes of CSF}

A: Average RNA amount in nanograms isolated from exosomes from $2 \mathrm{ml} \mathrm{CSF}$, (error bars represent SEM)

B: Correlations in miRNA expression between serial dilutions (5, 1, 0.5 and $0.25 \mathrm{ng}$ of CSF exosomal RNA) C: Inter- and intra-subject correlations from varying amount of CSF exosomal RNA. Color and size of the circles both represent degree of correlation. 
A

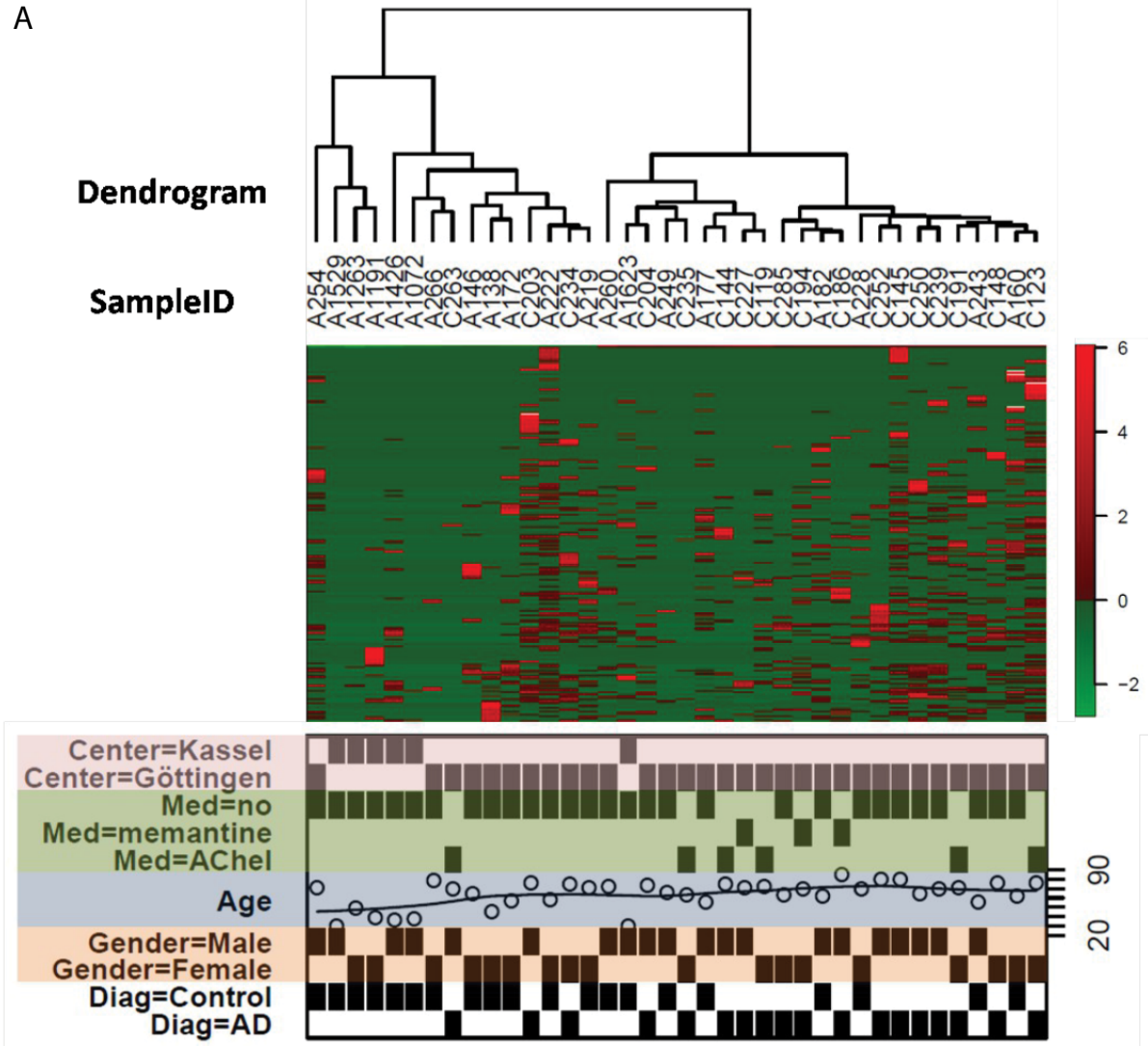

B

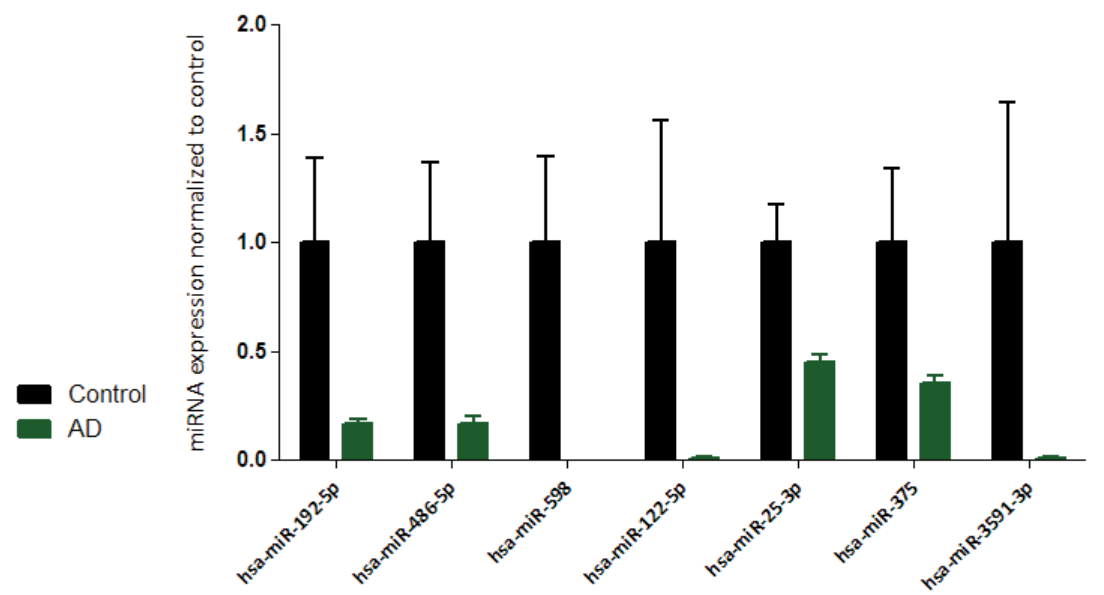

Figure 3.2.6.1 Differential expression and unsupervised clustering of CSF exosomal RNA from AD patients

A : Heatmap and dendrogram showing unsupervised hierarchical clustering of patients by CSF exosome microRNA expression profile. Each vertical row correspond $s$ to a single patient and the annotations at the bottom of the plot represent patient characteristics. The horizontal rows represent individual microRNAs and the color values represent microRNA expression levels (red signifies expression higher mean and green lower than mean) Med = medication, Achel = Acetyl cholinesterase inhibitor, Diag = diagnosis $\mathrm{B}$ : Differentially expressed microRNAs between AD patients $(n=18)$ and controls $(n=20)$. P values after adjusting for multiple testing are 4.13E-05, 5.53E-05, 0.000964, 0.019274, 0.024969 and 0.027931 respectively. Error bars represent standard error of mean. 


\subsubsection{Machine learning approach to predict AD}

The Random Forest machine learning algorithm was used to check if a combination of microRNAs could be selected that would allow a prediction of diagnosis based entirely on exosomal RNA. The set of 38 samples was divided randomly into a 'training set' of 28 (13 AD and 15 controls) and a 'testing set' of 10 (5 AD and 5 controls). Iterations of variable ranking by importance, estimation of accuracy by cross-validation and optimization of the number of microRNAs selected for the final model were performed on the training set. An internal accuracy rate of above $70 \%$ (out-of-the-bag error rate of $28 \%$ ), estimated by cross validation within the training set was achieved with a set of 25 microRNAs. As seen in Fig (3.2.6.2C) the lowest error rate in these data is obtained by using about 25 features. When the microRNAs were ranked by relative contribution to the prediction, the top microRNA, hsa-miR-34c appeared to contribute about 2-fold more than the other microRNAs (Figure 3.2.6.2B). Interestingly, the predictive microRNAs are not exactly the same as the most highly differentially expressed, although the 2 lists overlap. Finally, the model based on 25 selected microRNAs was tested on the testing set and a similar accuracy rate of $70 \%$ was achieved (Fig 3.2.6.2D).

Since the small fraction of the exosomal RNA (20-40nt) contains more than 3000 other non-coding RNAs, the non-coding RNA data were also used to attempt a prediction. Training and testing were performed in exactly the same manner as in Fig 3.2.6.2A for the AD patients and controls using a dataset consisting of other non-coding RNAs. Again, the lowest error rate by internal cross-validation within the training set was achieved at about 25 features (Fig 3.2.6.2F). When tested using the testing set, a higher accuracy rate of $80 \%$ was achieved (Fig 3.2.6.2G).

Finally, the 25 most predictive microRNAs and other non-coding RNA lists were combined to generate a combined dataset of 50 features, which was then trained and tested in a similar manner. The combination achieved an accuracy rate of 0.82 (2 errors out of 11 predictions) (Fig 3.2.6.2H).

For additional notes on the Random Forest algorithm and variable importance ranking, see Appendix 1

Figure 3.2.6.2 Machine learning approach to predict AD (Facing page)

Legend : A. Scheme used for training and testing the Random Forest algorithm, B; The top 10 microRNAs selected by the random forest algorithm as predictors of disease status in decreasing order of importance. Horizontal axis is represents decrease in Gini index (See appendix 2) C. Estimation of optimal number of microRNAs to be used for the prediction algorithm. On the horizontal axis are the number of features or microRNAs, and the vertical axis denotes the error rate estimated by cross-validation within the training set, D: Result of testing of the 25-microRNA model on the testing set, error rate $=3$ out of 10, E: Expression levels of the top 10 microRNAs in AD patients and controls plotted individually 
A

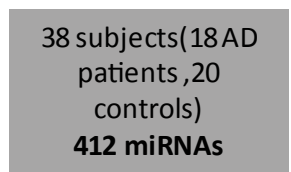

Test set (10 subjects)

$\because \bullet$

B

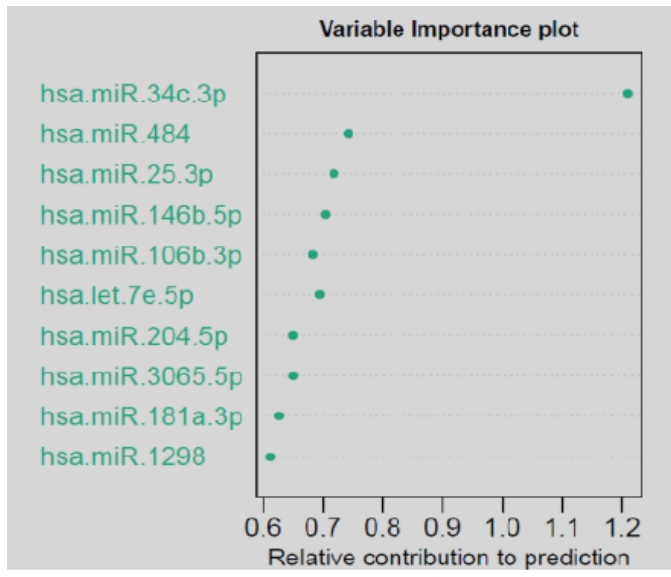

C

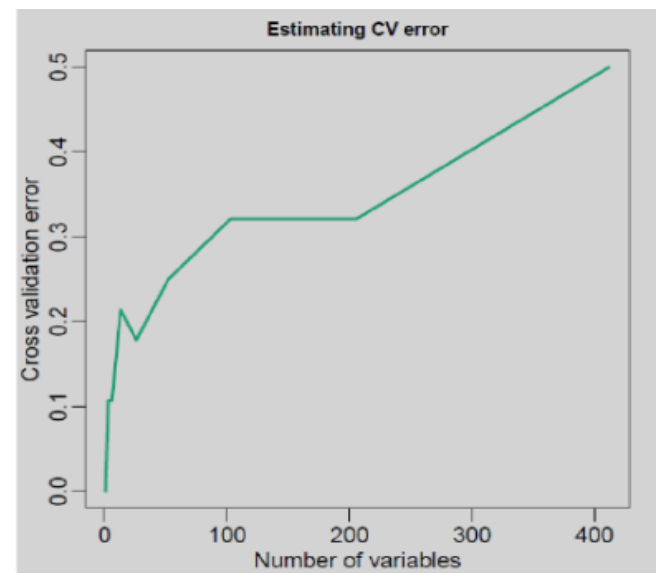

Final model

25 miRNAs
Cross-validate

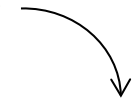

Primary estimation of accuracy

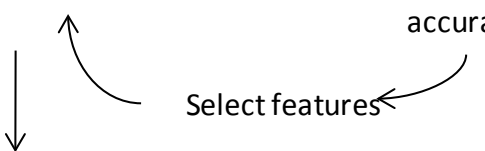

D

$$
\begin{array}{lllll}
\text { MiRNA } & 1 & 2 & 3 & 4
\end{array}
$$

56

Diagnosis Control Control Control Control Control $A D \quad A D \quad A D \quad A D \quad A D$

Predicted Diagnosis Control $A D$ Control Control Control $A D$ AD $A D$ Control Control CSF1263_A CSF222_A CSF254_A CSF1191_A CSF219_A CSF123_C CSF203_C CSF252_C CSF191_C CSF263_C

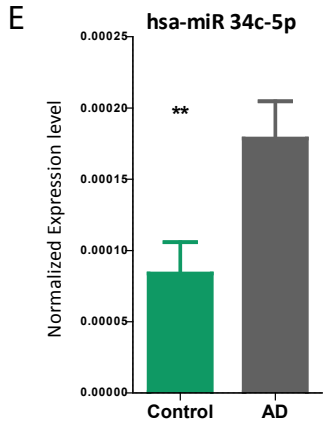

let $7 e 5 p$

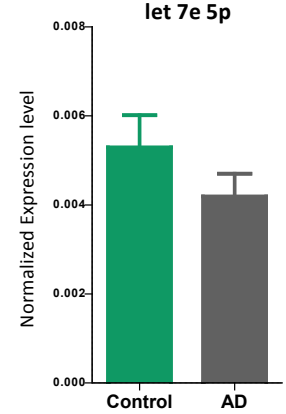

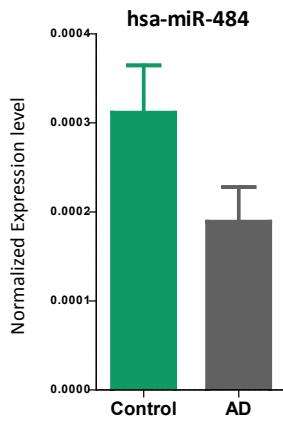

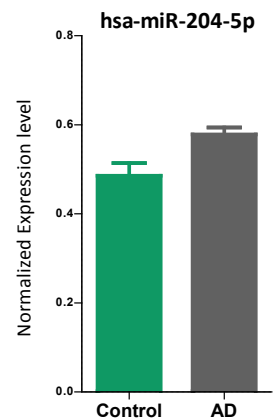

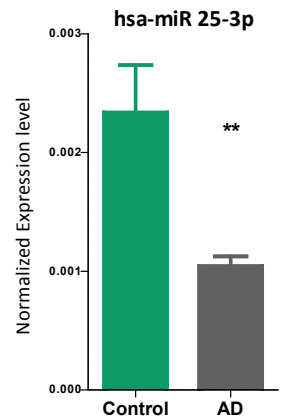

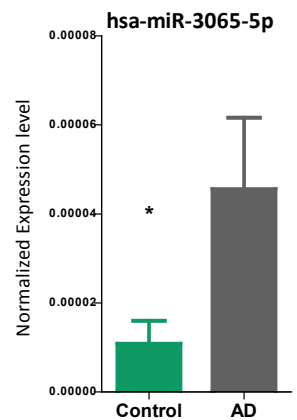

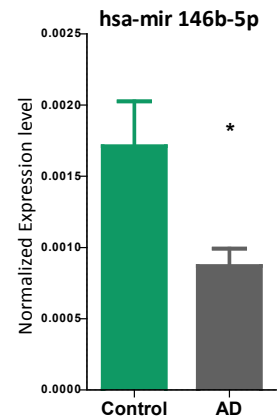

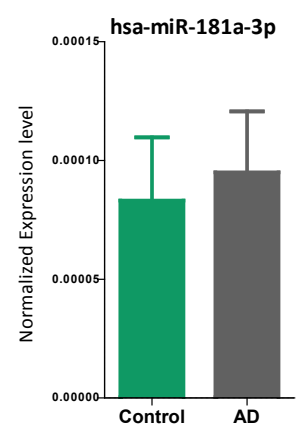

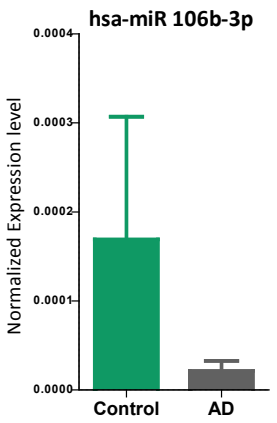

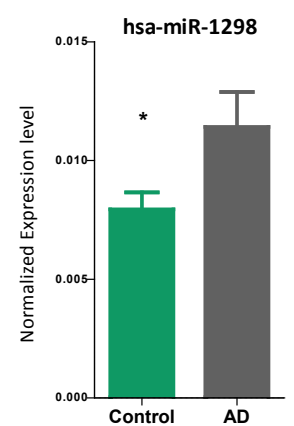




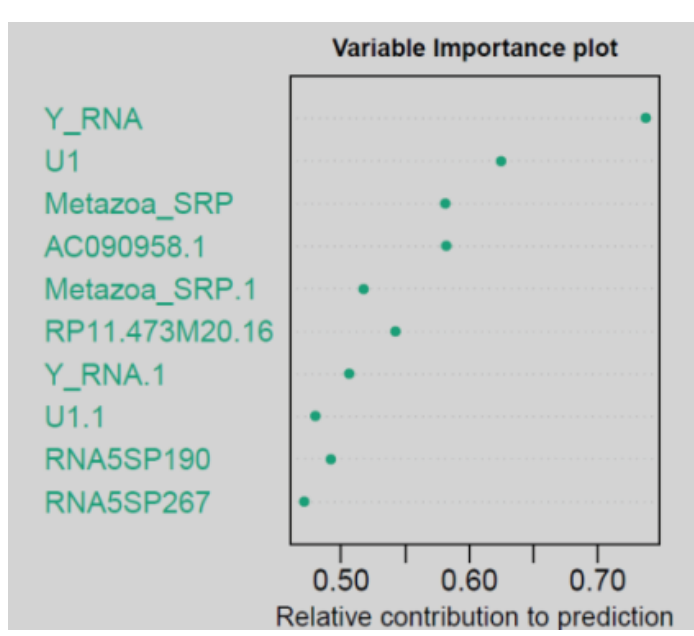

G

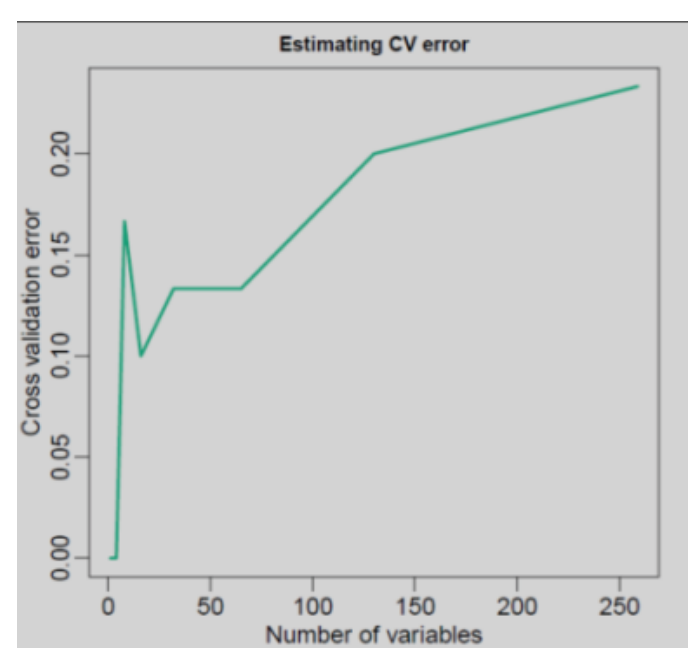

$\mathrm{H}$

miscRNA
Diagnosis Control Control Control Control Control

I

$\begin{array}{rrrrrrrrrrrr}\text { RF Combined } & 1 & 2 & 3 & 4 & 5 & 6 & 7 & 8 & 9 & 10 & 11 \\ \text { Diagnosis } & \text { Control Control Control Control Control Control } & \text { AD } & \text { AD } & \text { AD } & \text { AD } & \text { AD } \\ \text { Predicted Diagnosis } & \text { Control } & \text { Control } & \text { Control } & \text { Control } & \text { Control Control } & \text { AD } & \text { AD } & \text { AD } & \text { Control Control } \\ \text { CSF1072_A } & \text { CSF1191_A } & \text { CSF1263_A } & \text { CSF138_A } & \text { CSF1426_A } & \text { CSF146_A } & \text { CSF239_C } & \text { CSF250_C } & \text { CSF252_C } & \text { CSF263_C } & \text { CSF285_C }\end{array}$

J

\begin{tabular}{|c|c|c|c|c|c|c|c|c|c|c|c|}
\hline SVM Combined & 1 & 2 & 3 & 4 & 5 & 6 & 7 & 8 & 9 & 10 & 11 \\
\hline Diagnosis & Control & Control & Control & Control & Control & Control & $A D$ & $A D$ & $A D$ & $A D$ & $A D$ \\
\hline Predicted Diagnosis & Control & Control & Control & Control & $A D$ & Control & $A D$ & $A D$ & Control & $A D$ & $A D$ \\
\hline
\end{tabular}

\section{Figure 3.2.6.2 Machine learning approach to predict $A D$}

$\mathrm{F}$ : The top 10 other non-coding RNAs selected by the random forest algorithm as predictors of disease status in decreasing order of importance, G: Estimation of optimal number of other non-coding RNAs to be used for the prediction algorithm. On the horizontal axis are the number of features or other noncoding RNAs, and the vertical axis denotes the error rate estimated by cross-validation within the training set. H: Result of testing of the 25-other-non-coding-RNA model on the testing set, error rate $=2$ out of 10 , I: Result of testing the combined (MicroRNA and other non-coding RNA) 50-feature model on the testing set, error rate $=2$ out of 11 . J: Result of using an independent machine learning algorithm (Support vector machines) to test the combined (MicroRNA and other non-coding RNA) 50-feature model on the testing set, error rate $=2$ out of 11 . 

4. Discussion 



\subsection{Gene expression changes in age-associated memory impairment}

\subsubsection{Heterogeneity in aging}

The proportion of elderly people in the world population has risen considerably in the past few decades, and continues to increase [231]. This demographic trend was initially particularly pronounced in the Western world, but is starting to be seen as a worldwide phenomenon that includes the large population groups in the developing countries [232]. Aging not only increases susceptibility to cardiovascular, orthopedic, and malignant and other diseases, but is also the most important risk factor for diseases of the cognitive system such as Alzheimer's disease. This means that it is more crucial than ever to understand the biological mechanisms of aging, and to investigate ways to prevent and treat age-associated diseases.

Although aging affects populations as a whole, there is a marked variability in the extent to which individuals are susceptible to its detrimental effects. This variability can be seen between different species, between different individuals within a species and between different tissues or cell types within the organism [233]. Inter-individual variation in rates of aging can be attributed to a complex mixture of factors. From twin and family studies, it is clear that genetics plays an important role in determining individual susceptibility to age-related changes, as does maternally inherited mitochondrial DNA [233]. At the same time lifestyle factors, diet, exercise, and the cumulative effects of exposure to various other environmental stimuli also drive age-related changes, and the degree of the respective contributions is hard to quantify. Studying these environmental and epigenetic factors is especially important when trying to find mechanisms that are amenable to therapeutic or preventive intervention.

The process of aging affects multiple organ-systems in the human body. Some of these age-related changes like the graying of hair are relatively benign, while others have more serious consequences. One of the agerelated changes that contributes the most to functional decline in the elderly is the loss of cognitive function. It is well known that the age-related impairment specifically affects certain domains of cognition, such as the ability to learn and recall new information [234-236]. Studies in aging people show that learning and memory abilities of a group of aged individuals lie across a wide spectrum with some individuals retaining high performance in old age, while others decline at varying rates [237].

These observations have been reproduced in rodents, largely in rats, where it has been shown that aging is associated with increased variability on a number of tasks [238-240]. From the results in Fig 3.1.1 it is clear that a large inter-individual variability in age-associated memory decline can be seen in wild-type mice at the age of 16 months. Since the lifespan of a mouse is up to 24 months, the 16-month age corresponds to late middle age. The reasoning behind the choice of this age-group was that intra-group variability would peak at late-middle-age, and the group of mice would be likely to contain individuals with preserved learning and memory as well as those who showed early decline. By contrast, in a group of mice at an older age most of the animals would be expected to show impairment. In support of this, post-mortem analysis of the human frontal cortex has shown that the largest heterogeneity in gene expression occurs in the middle-tolate ages, with relatively less intra-group variability in the young, the middle-aged and the oldest patients [241]. As seen from Fig 3.1.2 the increased variability seen on the water maze task is not replicated in the fear conditioning and novel object recognition tasks. Since these tasks require different sets of cognitive 
skills and involve different brain regions (See Section 1.1.6 and 4.1.3) it is possible that they decline at different rates, and perhaps a different age group would show a wider variability in performance on these tasks.

Previous studies of the transcriptional changes in aging and age-related memory impairment have largely involved testing between 2 or more groups of animals at different ages [242-244]. Between-groups experimental designs are able to to identify the large groups of genes that are differentially expressed between young and old individuals. However they can not distinguish between benign age-associated changes and those that are associated specifically with the impairment of the function under study. The advantage of studying the spectrum of cognitive abilities using a single-group design is that the intragroup variability provides a means to separate the mechanisms associated with functional impairment from the background age-related changes. Additionally, it would perhaps allow a clearer demarcation between age-related changes that cause detrimental function and those that are part of a beneficial compensatory response. This provides a system for studying the underlying molecular changes that signal those subtle, cumulative insults that are associated with memory decline in aging.

\subsubsection{Distinguishing aging individuals with age-associated memory impairment}

Drawing inferences about the cognitive consequences of age-related molecular changes is not a straightforward process. As the aging process is associated with altered functioning, deterioration and potentially compensatory responses in many organ systems and cellular and molecular processes, there is a lot of room for confounding factors when making conclusions about the mechanisms driving agerelated cognitive decline. Some examples of these factors are the deterioration of the musculoskeletal and visual systems that could influence the interpretation of mouse behavior tests, and histopathological and molecular alterations that are associated with senescence but unrelated to cognition.

Although Fig 3.1.1 demonstrates increased variability in aged mice on the water maze task, it is not clear from these results whether the differences in performance are consistent between testing days. The water maze paradigm was ideal for demonstrating this, since it consists of several consecutive days of training and testing. A correlation analysis was therefore necessary in order to check whether the 'poor' or 'good' performance of the mice is more than an occasional phenomenon and to allow tracing of individual performance over time. The correlation tests in Fig 3.1.3 show that for all age groups, the level of performance remains relatively consistent over days. This analysis is also useful to distinguish good learners from poor learners for subsequent molecular analysis. Moreover, these results also distinguish inter-individual variability from intra-individual variability of inter-day fluctuations in performance. In our study, individuals were monitored simply be keeping them single housed for the duration of the experiments. A similar recently published study studying inter-individual variability in mice employed group housed mice with radio-frequency identification tags and antennae placed in the cage [245]. This study focused on exploratory behavior rather than learning or memory.

The spatial memory involved in the Morris water maze task is known to be primarily hippocampus-dependent [246] at the time points tested in this experiment. The fear conditioning task is known to involve both the amygdala and the hippocampus. While the amygdala is critical to the fear response [247], the contextual fear memory also involves the hippocampus [248]. 
In mice the recognition of novel objects depends on the rhinal cortex [249], but the novel object recognition task is a complex task that likely involves several other brain regions that encode and retrieve memory of the familiar object. Within the hippocampus, place cells are known to encode specific locations [250], and neuronal activity patterns that encode time and distance traveled by an animal [251]. The learning- and memory-associated activities involved in each of the tasks tested here require a combination of several different activity patterns.

Thus the demonstration that individual performance varies for different types of memory is not unexpected, and is further evidence for independent mechanisms in these types of learning.

\subsubsection{Comparison with previous rodent studies on age-associated memory impairment}

In order to dissect out the general mechanisms that accompany aging from those which specifically relate to age-related cognitive impairment, a series of studies was performed on aged rats, which show intragroup variability in the ability to learn spatial tasks [252]. In contrast to the work described in this thesis, where molecular correlations were drawn across a spectrum of behavior performance, the aged rats were divided into two groups - learning-impaired and learning-unimpaired [253-255]. By semi-quantititative immunohistochemistry, it was shown that the expression of the AMPA receptor subunit GluR1 and PSD-95 were increased in those rats that showed impairment with aging [255].

Studies in rats also show that animals that age successfully or retain their cognitive capabilities as they age do so by switching to an alternative mode of synaptic plasticity. While young animals use NMDA receptor dependent LTP and LTD, successful preservation of plasticity in aged animals is associated with a switch to NMDA-receptor- independent LTP and mGluR-dependent LTD [256].

The relationship between adult hippocampal neurogenesis and spatial learning in aged rats has also been investigated extensively, with somewhat contradictory results. Gene expression data from the dentate gyrus of these rats suggest that a decrease in expression of neurogenesis-related genes occurs uniformly with aging, and does not relate with learning impairment [257]. Studies of BrdU-positive cells (a marker for cell proliferation) in the hippocampus of aged rats showed that high BrdU-positivity in the hippocampal granule cell layer correlated with worse performance [258], or showed no correlation with performance [253]. Other studies with similar methodologies have shown that aged-unimpaired rats show higher levels of cell proliferation than aged-impaired rats [259].An isolated report suggesting impaired differentiation of newborn granule cells correlates with impaired learning [260] came under strong scrutiny, when a reevaluation of the correlations between the behavioral and immunofluorescence data showed the opposite to be true [261-263].

Interestingly, there are reports that markers of immature neurons are enhanced in patients with AD [264] and there is increased neurogenesis in the dentate gyrus and subventricular zone of a transgenic $A D$ mouse model [265]. 


\subsubsection{Ribosomes, translation and RNA-binding proteins in learning and memory}

The overlapping sets of genes coding for ribosomal proteins, RNA-binding proteins and the translational machinery show a correlation with memory performance in old age. Aging mice expressing high levels of these genes in the hippocampus perform better than average on the water maze task. From Fig 3.1.6.1 and 3.1.6.2 it is clear that the entire translation initiation pathway is expressed at high levels in these animals. The control of plasticity at the level of translation is a well known phenomenon and many components of these pathways have been studied in detail with respect to learning and memory

\subsubsection{RNA-binding proteins, RNA transport and activity-dependent local translation}

RNA-binding proteins (RBPs) are a group of proteins that form complexes with RNA in the cellular nucleus or cytoplasm. The nuclear RBPs mostly form complexes with pre-mRNA, called heterogeneous ribonucleoprotein particles but the cytoplasmic RBPs are more diverse and are known to carry out a variety of functions. Some of the functions that have been ascribed to RBPs include export of RNA from the nucleus into the cytoplasm, regulation of splicing and alternative splicing, RNA editing, and polyadenylation of mRNA transcripts, and constraining their translation [266]. Thus they are key regulators of postranscriptional processing of RNA. One particularly interesting function of RBPs with respect to neurons is their role controlling RNA localization, particularly in regulating mRNA transport to the site of local translation [266].

The RNA-binding protein FMR1, or the fragile $X$ mental retardation protein 1 gene was found in the gene set that correlated with good performance. FMR protein (FMRP) plays a role in synaptic plasticity and activity-dependent translation at the synapse and is key for mGluR-dependent LTD. FMRP is involved in activity-dependent mRNA transport in neurons [267-269]. This protein has also been shown to be a translation inhibitor, repressing activity-dependent translation [270], and to inhibit the translation of specific mRNAs at the synapse [271]. Further, there is strong evidence for a relationship between FMR1 and glutamate receptors. The translation of Fmr1 and the localization of the mRNA to dendrites is regulated by activation of group 1 metabotropic glutamate receptors (mGluRs) [269]. At the synapse, mGluR activation however, leads to degradation of the FMR protein, [272]. In mice where activity-dependent FMR translation is impaired, mGluR-mediated long-term depression (LTD) is enhanced. Stau2, and Hnrnpa2b1 are other genes on the list that are also known to be closely associated with the RNA granules that are transported to the dendrites as a part of local protein synthesis [273, 274].

\subsubsection{Insulin signaling, mTOR and translational regulation}

Eukaryotic Initiation Factor 2 (elF2) and polyA binding protein, twp of the genes whose expression correlated most highly with behavior performance in old age are key players in the initiation of mRNA translation. As shown in Fig 3.1.6.2 elF2 binds guanidine triphosphate and the initial methionine-tRNA before forming a complex with the other translation initiation factors elF1, elF2A and elF3. The polyA binding protein (PAPB), another important component of the translational machinery also appears to be regulated in the same direction as the elFs. 
Many of the genes coding for ribosomal proteins, as also the translation initiation factors are regulated by mTOR. There is a large body of evidence pointing to the involvement of the mammalian target of rapamycin (mTOR) in neuronal translation regulation [275]. mTOR couples the activity of neuronal surface receptors to the protein synthesis required for synaptic plasticity. The mTOR inhibitor rapamycin acts by disrupting the mTOR protein complex (MTORc) [276]. High levels of mTORc1 repress the elF4E-binding proteins (4E-BPs). Rapamycin-sensitive mTOR signaling is essential to protein synthesis-dependent synaptic plasticity [277]. Moreover, mTOR signaling is also essential to mGLuR-dependent neuronal LTD (distinct from N-methyl-D-aspartate receptor (NMDAR)-dependent LTD) [278].

In a rat model of accelerated aging, the mTOR inhibitor rapamycin allayed cognitive deficits and prevented the onset of structural brain abnormalities associated with senescence [279]. Inhibition of the mTOR complex by rapamycin was shown to increase lifespan in both genders and in wild type mice with different genetic backgrounds [280]. In human studies, there is preliminary evidence that treatment with mTOR inhibitors could lead to improvement in memory and concentration, among other psychiatric symptoms [281].

Although it has been established that synaptic activity causes mTOR to interact with and regulates translation initiation factors and $\mathrm{S} 6 \mathrm{~K}$, it does not universally upregulate translation at the activated synapse. Increased mTOR activity specifically downregulates the expression of proteins such as IRS2 (Insulin receptor substrate 2) and dendritic translation of voltage gated potassium channel 1.1(Kv1.1) [282]. Interestingly, comparing the expression of genes within the translation initiation pathway between good learners and poor learners (Fig 3.1.6.2) suggests that the expression of insulin and components in the insulin-signaling pathways may be important events in the process.

\subsubsection{Glutamate receptors, excitotoxicity and memory impairment}

From the results of the gene set enrichment analysis and the more detailed gene-level analysis in sections 3.1.5 and 3.1.6 it appears that high hippocampal expression of components of the glutamate signaling pathway correlates with poor memory performance in old age. These components include a number of postsynaptic glutamate receptor subunits, but overexpression at the gene expression level can not be directly interpreted as heightened glutamatergic signaling at the synapse. Moreover while these changes in glutamate receptor subunit expression certainly indicate a connection between glutamate signaling at the synapse and the maintenance of memory in old age, it is not clear whether high levels of glutamate receptors are a cause or a consequence of the pathology. Interestingly, the transcription of metabotropic glutamate receptors as well as some of the ionotropic glutamate receptors seem to change concurrently.

The relationship between excessive glutamatergic stimulation and neurodegeneration has been demonstrated in several neurological systems [283-285], and the term 'excitotoxicity' was coined to describe it [284]. Glutamate is the major excitatory neurotransmitter in the mammalian CNS, but prolonged excess glutamatergic stimulation causes neuronal apoptosis [286, 287]. This phenomenon has been shown to play a role in neurodegeneration not only in $A D[288,289]$ but also in models of Parkinson's disease [290], brain and spinal cord injury [291], multiple sclerosis [285] and amyotrophic lateral sclerosis [292]. The NMDA receptor blocker Memantine, one of the most commonly prescribed drugs to treat Alzheimer's disease is based on the attenuation of glutamatergic signaling [293, 294].

Thus increased glutamate stimulation has been shown to be linked especially with NMDA-type glutamate 
receptors. In our paradigm however, we see a concommitant upregulation of all types of glutamate receptors with the changes in metabotropic glutamate receptors (mGluRs) being most prominent.

\subsection{RNA in the study of Alzheimer's disease}

\subsubsection{Gene and microRNA expression in mouse models of brain amyloidosis}

Since the molecular mechanisms in human AD can only be studied post-mortem, a number of studies have been constructed in mouse models that overexpress amyloid-beta and in cell culture after addition of amyloidbeta. We used one of these models, the APP-PS1 double transgenic model of amyloid overexpression to study the mRNA and microRNA expression changes. The results in Fig 3.2.1 show a small significant change in microRNAs during early disease, and fewer changes at an older age group. A study similar to ours, performed in the APP23 mouse line, a single transgenic line that overexpresses mutant human APP also showed evidence of microRNA dysregulation [295]. In these mice, which develop amyloid plaques later than the double transgenic mice we used, hippocampal expression of 9 microRNAs was studied by qPCR at various ages. the authors reported a downregulation of nearly all tested microRNAs (mmu-miR-9, $-181 c,-409-3 p,-361,148 b,-21$,-let-7i, and -30c) at 7-8 months of age, and a return to a relatively normal microRNA profile at later ages.

Interestingly, 2 of the 6 microRNAs that we found to be downregulated in the APP-PS1 mice (mmu-miR-9 and mmu-miR-409c) overlap with the list previously generated [295] in the single transgenic mice. Further the timing of microRNA dysregulation with respect to plaque forming age is also similar. The peak in microRNA expression abnormalities appears to occur relatively early in the pathogenesis, just before the appearance of large, distinct plaques and behavioral manifestations. A previous microarray study in the cerebral cortex of another double transgenic APP-expressing strain also showed evidence of disturbance in microRNA levels, with 37 microRNAs being differentially expressed, including downregulation of mmu-miR-29a and $-106 \mathrm{~b}$ and upregulation of miR-34a [296]. This study also showed a peak in microRNA deregulation during the peak plaque-forming age, when soluble A-beta levels are highest, before large and extensive plaques developed.

Aside from the two microRNAs mentioned above, the list of differentially expressed microRNAs in the APP-PS1 double transgenic mice differs from the list obtained by the previous study. There is more than one possible reason for this discrepancy between the results. The mouse strain we use develops plaques and cognitive symptoms earlier than the APP23 strain, and it is possible that the ages used (4 months and 8 months) are too late to capture the early disruption in miRNA levels. The methodology used also differs between the 2 studies; our study is a genome-wide profiling, while Schonrock et al. use a targeted approach. The relatively stringent correction for multiple testing used in our NGS study could have caused a lowering of sensitivity to small changes in microRNA expression.

Aside from these studies, the other investigations into microRNA disruption caused by A-beta have been conducted in vitro. The microRNAs hsa-miR-106a, -520c [297], -101 [298, 299], members of the miR-20a family [300], and miR-655, -323-3p, -644, -153 [301] all reportedly target APP through binding sites on its $3^{\prime}$ UTR. MiR-124, the most abundantly expressed microRNA in human neuronal cells, has also been 
suggested to play a role in the regulation alternative splicing of APP mRNA. MicroRNAs may also indirectly regulate levels of amyloid by targeting the beta-secretase BACE. MicroRNAs hsa-miR-29a, $-29 \mathrm{~b}-1$, and -9 show an inverse correlation with BACE protein levels in AD patients, and in a cell culture overexpressing mutated APP, they were able to suppress the production of A-beta peptides [302]. Evidence from mouse models, however, disputes the link between miR29a/b and BACE [303]. APP and amyloid have also been shown to cause downstream effects on microRNA networks. Adding fibrillar A-beta42 to primary neurons was shown to cause alterations in a large number of microRNAs in both directions [295].

Although A-beta has a strong link with $A D$, human $A D$ is associated with several other pathomechanisms, which are not recapitulated by overexpression of APP or treatment with A-Beta [303]. Since the development of a mouse model that replicates the tau pathology and all the other characteristics of $A D$ in addition to the A-beta has proved difficult, microRNA studies on the AD-afflicted brain have thus far have largely been limited to postmortem analyses in humans. A qPCR array in different brain regions of AD patients at various Braak stages showed that the expression of several microRNAs such as hsa-miR-200c, -212 , $-26 a,-27 a,-30 c,-30 e 5 p,-34 a,-381,-422 a,-423,-9,-92$, ( $p$ value $<0.05$, no correction for multiple testing) [304] changed with advancing AD. Subsequently, microarray analysis of the AD parietal cortex showed 40 differentially expressed microRNAs, the top 10 being miR-30184, -617, -101, -06383, -10912, -20546, $-601,29 \mathrm{~b},-181 \mathrm{c}$ and -23974 (multiple testing correction used, FDR < 0.05) [305]. The most recent study of microRNA changes in the AD brain used an nCounter (Nanostring technologies) method to show the deregulation of 35 miRNAs in the hippocampus of patients with sporadic AD [306].

Other studies focusing on specific microRNAs have shown changes in expression of the miR-29a/b family [302, 307], miR-107 [308, 309], miR-9 and miR-128 [310]. Thus a large number of microRNAs have been reported as differentially expressed, but the overlap between these studies is relatively small (Fig 4.2.1). Within the set of overlapping miRNAs are hsa-miR-132, hsa-miR 425, hsa-miR-200, hsa-miR-27, hsamiR-9, hsa-miR-29a/b and miR-107, which have all been reported in more than one study as being altered in AD [311]. Interestingly, there is some disagreement between studies over the direction of dysregulation. While Lukiw et al. [310] report hsa-miR-9 and hsa-miR-128 to be upregulated in the hippocampus of patients with $A D$, these microRNAs have been reported as downregulated in the AD hippocampus in other studies $[304,306]$

A direct comparison of all the studies is somewhat difficult, because they have been conducted in different brain regions. Fig 4.2.1 shows an overview of the overlap between various human studies on miRNA changes in the $A D$ hippocampus. It can also be seen that there is little overlap between the microRNA changes in the human $A D$ hippocampus and those in the hippocampus of transgenic amyloid mouse models.

Thus using the currently available methods of investigation, no consensus has emerged about the role that microRNAs play in disease, and evidence from living patients or patients in early stages of AD is lacking. 
Human AD hippocampus studies

Cogswell et al. 2008 (11up, 7 down)

Lau, de Strooper et al. et al. 2013 (11up, 7 down)
APP mouse hippocampus studies

APP-PS1- Section 3.2.1 (6 up 10 down)

Schon rock et al. 2013, (1up, 8 down)
A

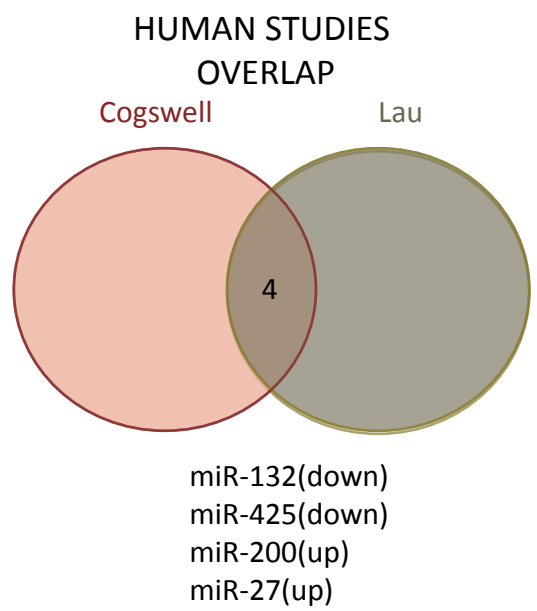

B MOUSE STUDIES OVERLAP

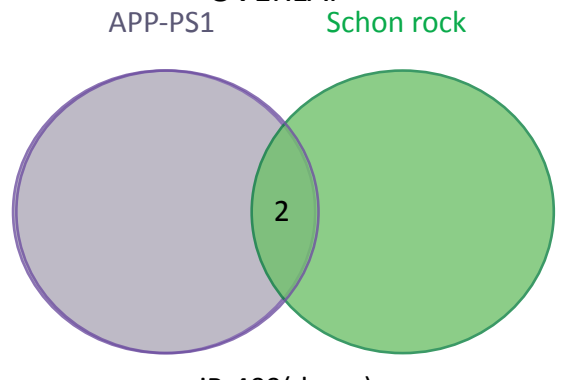

miR-409(down) miR-9(down)

\section{OVERLAP BETWEEN HUMAN AND MOUSE STUDIES}

C

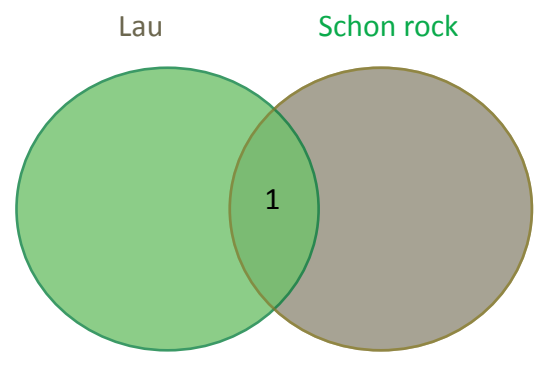

miR-409(down)

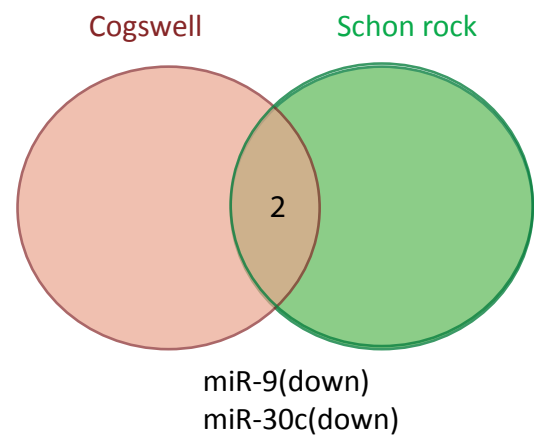

Figure 4.2.1 Comparison between previous human and mouse microRNA studies of AD

A: Overlap between differentially expressed microRNAs in 2 major genome-wide human studies of the human AD hippocampus, B: Overlap between differentially expressed microRNAs in the hippocampus of the APP-PS1 mouse model (Section 3.2.1) and the APP mouse model(Schonrock et al. 2010) C and D: Overlap between differentially expressed microRNAs in mouse and human studies. 


\subsubsection{Feasibility of RNA isolation from CSF}

As discussed in the section above, the methods for studying RNA profiles in the early stages of Alzheimer's disease are limited. Thus the possibility of studying brain-derived RNA-containing vesicles would provide a new insight into the pathomechanisms at all stages of disease. However, until recently it was not clear that RNA exists in a stable form outside cells. One of the earliest descriptions of RNA, published in the 1960s describes it as an "unstable intermediate for carrying information from genes to ribosomes"[312]. Since then, and after the currently used standard method for RNA isolation, by guanidium thiocyanatephenol-chloroform (TRIzol or TRI-reagent) [313, 314], was established for extraction of RNA from plant and animal tissues, the process of RNA isolation is still generally known to require great care to protect RNA from degradation by ubiquitously occurring RNases. It has been generally held that cellular RNA is protected from degradation by special intracellular conditions and while being isolated from the cell, it needs additional protection from RNases. This emphasis on the instability of RNA was perhaps responsible for the long-held assumption that non-degraded RNA can only be isolated from intact cells and tissue.

This assumption has recently been challenged. From Fig 3.2.2 as well as recently published literature (See section 1.2.4) it is now clear that RNA can be successfully isolated from cell-free biological fluids such as cell culture media, CSF and plasma. There are several mechanisms such as encapsulation in vesicles or lipoprotein complexes now known to protect RNA, especially microRNA, from degradation when it is circulating in extracellular fluid. While microRNAs in plasma have been shown to be especially resistant to endogenous RNAse degradation [315], from our observation and those of others [316, 317], it is evident that mRNAs can also be extracted from body fluids with a high degree of reproducibility.

The results we obtained regarding the RNA contents of the vesicular fractions of CSF were since confirmed in extracellular fluid from cell culture. A study comparing the RNA content of apoptotic bodies, microvesicles and exosomal vesicles isolated from 3 different cultured cell types established that the 3 kinds of vesicles have distinct RNA profiles. Apoptotic bodies have a high content of ribosomal RNA and their RNA profile resembles the cellular RNA profile, microvesicles contain little or no RNA, and exosomes are enriched in small RNA [318]. Though the highest number of microRNAs in our study are seen in the exosomes (around 150 ), this number is far lower than the number of microRNAs that can be detected from human brain cells. However, evidence from neuronal cultures (Fig 3.2.4) suggests that exosomes carry a repertoire of microRNAs that is comparable to that in the cells. Thus the relatively low number of microRNAs isolated in exosomes points to the need for a more sensitive technique to preserve, isolate and detect RNA from CSF. It is possible that the exosome-free fraction of CSF contains RNA bound to proteins or lipoproteins, that are not efficiently extracted by the method we used. Another possibility is that a part of the RNA is suffers degradation during the exosome isolation procedure. Around the time that this thesis was written an NGS study of CSF microRNA detected 486 microRNAs in total, unfractionated CSF [319].

\subsubsection{Choice of methods for RNA isolation from CSF}

However the isolation of RNA from extracellular fluids is complicated by the availability and widespread use of a large number of different RNA isolation methods and proprietary commercial reagents. The differences between these methods are at the levels of lysis reagent (guanidium thiocyanate-phenol-chloroform, 
detergents or a combination) and RNA precipitation (simple precipitation or elution after precipitation on a column). In the case of exosomal RNA and other circulating RNA, the choice of RNA isolation method appears to be of particular importance. A study comparing various RNA isolation methods used on exosomes from cultured cells showed that not only the quantity but also the size profile of the isolated RNA differed based on the methods used [320]. Other studies comparing methods for the isolation of circulating RNA reported that the methods that lead to larger total RNA yields are not necessarily those that yield the best detection of microRNAS [319], indicating that some of these methods may be selective for certain RNA populations. However, that the results of these comparative studies cannot be generalized, and sometimes conflict with one another. While one study showed that the Exiqon miRCURY Biofluids Kit as the bestperforming method to isolate RNA from circulating body fluids [321], a simultaneous one that used this kit [322] reported the inability to isolate and detect microRNAs from CSF that were detected by us using the TRI-Reagent.

All in all, it appears that reproducibility of the results depends quite heavily on the choice of RNA isolation methods. For this reason, we chose the TRI-Reagent method over commercial RNA isolation kits with undisclosed proprietary reagents, and used this method consistently for all RNA isolation from fluids, cells and tissue.

\subsubsection{RNA species in CSF exosomes}

Though exosomes are derived from cells, their RNA profile is distinct from the cellular profile. Based on our results and those from other published studies, exosomes appear to be enriched in RNA below $200 \mathrm{nt}$ in size and are rich in non-coding RNA.

\subsubsection{Small RNA enrichment in CSF exosomes}

The size profile of the RNA we isolated from CSF exosomes matches that described in the literature for RNA isolated from various cell types and biological fluids [323-326]. The repeated finding of a large peak in the small RNA region, and the virtual absence of RNA species above 200nt in length raises several questions, as does the absence of the ribosomal RNA subunits that dominate the cellular RNA profile. One possibility is that the observed profile represents degraded cellular RNA, with the larger RNAs being degraded and the smaller ones being more resistant to degradation. However, the consistent well-defined nature of the small RNA peak suggests otherwise. The other possibility is that the cells apply a machinery that targets specific small RNAs to exosomes.

A closer look at the size distribution of exosomal RNA reveals that this peak is not in the mature microRNA region (22-26 nt), but lies between 100-150 nt in exosomes from CSF, plasma, and cell culture. While exosomes clearly contain numerous microRNAS and mRNAs (Fig 3.2.3.2 and 3.2.3.3), the other RNA contents of the exosomes remain to be explored. Next-generation sequencing (NGS), a high throughput method which can be used to reconstruct RNA sequences before mapping them to the genome could thus prove highly valuable for this purpose. In this context, one particular advantage of NGS over arraybased technology is that we are not limited to the known and well-annotated species such as mRNAs and 
microRNAs but can potentially study the lesser known non-coding RNAs.

The first study to sequence the contents of exosomes was performed in cultured immune dendritic cells [327]. This study presumed that the peak consisted of tRNAs and excluded it from their sequencing protocol, only sequencing RNA species below 70nt in length. However, tRNAs are commonly known to be 70-95nt in length, and would not fully explain the observed RNA profile. A similar study in exosomes from prioninfected neuronal cells, also selectively sequenced the RNAs below 40nt in length, labeling the higher peak as tRNA [328].

A study of RNA in exosomes derived from saliva [329] and one of microparticles circulating in plasma sequenced the entire 20-200nt region, but mapped and reported only the microRNA contents [330]. Between the publication of the results of the first sequencing of exosomal contents in 2012 and the writing of this thesis, there have also been several qPCR- and array-based investigations published into RNA contents of the exosome, focusing mostly on the microRNAs and mRNAs [329]. It has been shown in at least one cell type that microRNAs are relatively depleted in exosomes compared to cells [328], and the bioanalyzer profile of exosomal RNA suggests the abundant presence of other RNA species below 200nt. Some possibilities that could lie in this region are 5S ribosomal RNA, tRNA, pri-microRNAs and a host of other non-coding RNAs about which little is known. Several non-coding RNAs below $75 \mathrm{nt}$ in length have been found such as Y RNAs, vault RNAs, specific t-RNA repeat regions, and other repeat regions [327]. There is also evidence that microvesicles secreted from glioblastoma cells contain retrotransposon sequences and other repetitive elements, with some of them such as Alu-1 and L1 being highly enriched in the vesicles over the cells [331]. It remains to be seen if exosomes from non-malignant cells also contain retrotransposon elements, but the possibility raises interesting questions about the role that extracellular vesicles play in intercellular information transfer. Thus there is still much to be studied about the exosomal RNA content beyond the microRNA and protein-coding transcripts.

However, the mapping of these non-coding RNAs to the genome after sequencing poses a significant challenge, with few established methods to perform this task. Little is known about the physiological function of many of these RNA types and the annotation databases for other non-coding RNA microRNA are relatively new and still not as comprehensive as those for mRNA or miRNA. The nature of the repeat sequences also makes their alignment to the genome technically more difficult.

\subsubsection{MicroRNA contents of CSF exosomes}

Our results represent the first sequencing study of exosomes from cerebrospinal fluid. RNA and microRNA has previously been identified in the CSF by qPCR [304, 322], microarray [332] in smaller scale studies, where all fractions of CSF RNA (cell debris-, vesicle- and lipoprotein-associated RNA) were pooled together. Around the time that the work described in this thesis was completed, the first NGS profiling of plasma and cerebrospinal fluid was published [319]. The number of microRNAs we detected in CSF exosomes was comparable with the number detected by this profiling study of whole CSF. However, the microRNA profile we detected in exosomes was not very similar to the microRNA profile detected in whole CSF. For example, the most abundant microRNA in CSF exosomes was hsa-miR-204, while the top miRNA in whole CSF was hsa-miR-486 [319]. Interestingly, hsa-miR-486 is the most abundant microRNA in plasma exosomes. These results suggest that the CSF exosomes contain a distinct set of microRNAs. 
The finding that exosomes are slightly depleted in microRNA as compared to their cells of origin replicates published observations in immune dendritic cells [327]. Similarly, the selective enrichment and depletion of certain microRNAs in exosomes has also been confirmed recently in a study of exosomes from a neuronal cell line [328]. While it is easy to imagine that certain cellular microRNAs may not be exported into exosomes, the presence of microRNAs in exosomes that are not detectable in cells is hard to explain. This finding is thus most likely attributable to the detection limits of the method used.

\subsubsection{Other non-coding RNA in CSF exosomes}

One of the better known long noncoding RNA highly expressed in the CSF exosomes is Neat1 or NuclearEnriched abundant transcript1. Neat1 RNA is essential for the formation of specialized subcellular structures called paraspeckles [333]. Paraspeckles are dynamic transcription-dependent granules that contain ribonucleoprotein and RNA [334]. Neat1 is thought to play a role in preventing inappropriate translation and regulating nuclear export of RNA containing inverted repeated Alu elements. The presence of Neat1 in exosomes, which have no known connection with the nucleus is thus interesting. Neat1 and other paraspeckle-related genes were also found to be strikingly upregulated in the hippocampus of aged mice [335] (unpublished).

Among the smaller non-coding RNAs, in the 40-200 nt regions, the most abundant transcripts belong to the Y RNA family. Y RNAs, which are RNA polymerase III transcripts have been shown to be highly enriched in extracellular vesicles from cultured cells [327]. They bind to the Ro ribonucleoprotein and have been studied mostly in the context of systemic lupus erythematosus.

In one of the samples (Sample 2), the most abundant transcripts are from the U RNA family of small nuclear RNAs. The U RNAs, a family of RNAs associated with the spliceosomal complex, and bind closely to small nuclear ribonucleoproteins. Interestingly, the U1 ribonucleoproteins have been recently shown to play a role in AD, perhaps through dysregulation of RNA splicing [336]. Thus the most abundant known noncoding RNAs from both the smaller and larger fractions of CSF exosomal RNA are associated with nuclear complexes, raising the question of how they are trafficked from the nucleus into exosomes.

\subsubsection{Exosomal RNA as an indicator of intracellular changes}

The overall finding that the exosomal RNA profile does not replicate that of the cell that it originates from is interesting because it demonstrates that the RNA secretion into exosomes is not a passive process, but rather a non-random selective one. Thus the excretion of RNA by the cell into exosomes might serve a functional purpose. However, it is not yet clear whether this purpose is the excretion of unwanted RNA intended for degradation or intercellular communication by RNA transport or both.

The results in Fig 3.2.4 indicate that the profile of microRNAs exported in exosomes changes during cellular response to insults. The distinct unsupervised separation between exosomes from untreated cells and exosome from A-beta-treated cells suggests that at least under carefully controlled in vitro conditions, 
exosomal RNA can be used to indicate the status of the neurons they originate from. The situation in a biological fluid like CSF or plasma is likely to be complicated by the fact that exosomes isolated from these fluid are likely to originate from a multitude of sources, with no currently available means to determine the individual contribution of each cell type to the exosome population.

The treatment of primary neurons with A-beta is a method notorious for its high variability from culture to culture and small differences in A-beta preparation methods are known to affect the results unpredictably. Further, the number of replicates used here is admittedly too small to allow extrapolation about the general effects of A-beta on neuronal or exosomal microRNA profiles. Yet, the list of differentially expressed neuronal microRNAs obtained after A-beta treatment under the conditions we used shows some overlap with a previous study of A-beta induced deregulation of microRNAs in hippocampal neuron cultures.

The opposite-direction shifts in the proportion of small non-coding RNA and long RNA in cells and exosomes, is interesting and appears to suggest that cells under stress by A-beta toxicity excrete more mRNA and long noncoding RNA into exosomes. Confirming these shifts and further investigating the selective secretion of certain RNA classes into exosomes would require a time course profiling of RNA in cells and exosomes after A-beta perturbation. While these experiments could potentially provide further insights into exosomecell relationships, they are not within the scope of the currently described work.

\subsubsection{Potential for use of CSF RNA as a biomarker for AD}

The random forest machine learning algorithm we used not only gives an estimate of prediction accuracy but also ranks variables based on their relative contribution to differentiating between groups. Based on the measure of variable importance used by the algorithm, hsa-miR-34c appears to be the microRNA with the highest power in differentiating between $A D$ patients and controls. At the level of the individual miRNA, miR-34c is also significantly higher in AD patients than controls. When the effect of age on the levels of this microRNA was examined, miR-34c showed a trend towards increase with age, but no significant difference between young and old groups. These results fit well with the published literature. MiR-34c has been shown before to be increased in the hippocampus of patients with AD [226]. A recent examination of microRNA levels in the hippocampus of patients with $A D$, showed that this increase was specific to the early stages of $A D$ [322]. Further evidence for the involvement of $34 \mathrm{c}$ in $A D$ and age-associated memory impairment comes from a mouse study showing that inhibition of $34 \mathrm{c}$ can rescue amyloid-induced as well as aginginduced learning deficits [226].

Since age is the biggest risk factor for $A D$, the age-related changes in miRNA expression are hard to separate completely from those associated with disease. In view of the published literature with regard to this particular microRNA, these results can be interpreted as meaning that $A D$ is associated with a large increase in miR-34c on a background of a smaller increase with aging. An alternative possibility is that $34 \mathrm{c}$ marks accelerated aging in the brain, and signifies an enhanced risk for AD. In either case, the results validate the potential of CSF exosomal microRNA as a method to study molecular pathophysiology in the living $A D$ brain.

Among the other microRNAs that seemed to differentiate between groups included miR-146b, which has been shown before to be downregulated in the human AD hippocampus [304] and miR-181a-3p. Thus, 
although more than one of these microRNAs is significantly different between groups, none of them is individually able to accurately predict disease status. Interestingly, some of the top predictive microRNAs were not significantly differentially expressed between groups, and the most significantly differentially expressed microRNA (miR-598) was not among the most predictive microRNAs. It is conceivable that a combination of up- and down-regulation patterns of a set of microRNAs together indicate an underlying pathological process. In such a situation, a learning algorithm is clearly more suited to the task of developing a 'disease signature' than manually selecting microRNAs based on differential expression between groups. 
Conclusions 



\subsubsection{RNA in age-associated memory impairment}

The work described in section 2.1 aimed to identify the molecular mechanisms that distinguish healthy cognitive aging from age-associated memory impairment. Based on these experiments we concluded that - As in humans, aging is associated with a decrease in performance on learning and memory tasks in mice. This decline does not occur at a uniform rate, but varies greatly from individual to individual. Thus there is high intra-group variability in age-associated memory decline.

- $\quad$ The water maze spatial learning task provides a useful model to study this heterogeneity in aging wild-type mice and can be used to distinguish good learners from poor learners within a group.

- $\quad$ The expression levels of a large number of genes correlate with water maze performance in aging mice. Analyzing these data by the GSEA technique defines sets of genes in the pathways associated with ribosomes and translation to be correlated with good performance. Conversely, the high expression of genes in the glutamate signaling pathway were found to be associated with poor performance.

- $\quad$ Comparing the levels of gene expression in the 3 best-performing individuals with the 3 worstperforming individuals confirmed that genes in the pathway associated with translation initiation are expressed at higher-than-average levels in the aging mice with good memory skills. Similarly, the 3 worstperforming mice expressed glutamate receptors and other components of the glutamate signaling pathway at higher-than average levels.

\subsubsection{CSF exosomal RNA in the study of AD}

The experiments described in section 2.2 were designed to establish and test a novel method of studying the molecular mechanisms in the brain of live patients with Alzheimer's disease. Extracellular vesicles from cerebrospinal fluid were isolated and used as sources of RNA to provide an insight into the cells they originated from.

- $\quad$ This work shows that CSF contains stable RNA species that can be reproducibly isolated and quantified, and that when using the methods described, the majority of RNA detected in CSF is in exosomal vesicles or is associated with them.

- Using next-generation sequencing to study the RNA contents of CSF exosomes revealed that a small fraction of the RNA was microRNA, another small fraction consisted of mRNA and a large proportion of the RNA was other non-coding RNA.

- The list of RNA transcripts in exosomes overlaps the list of transcripts in the neurons from which it originates, with certain RNA and microRNA species being enriched in the exosome. Perturbation of the cell by amyloid-beta treatment is reflected clearly by a change in the exosomal RNA content, but the exosomal RNA profile is distinct from the cellular RNA profile.

- Increase in CSF exosomal levels of microRNA 34c were detected by an unbiased screen of exosomal RNA to be associated with Alzheimer's disease. Interestingly, this microRNA has been linked to $A D$ by both animal and human studies before. This finding shows that studying CSF could be a method to detect more coding and non-coding RNAs involved in AD.

- $\quad$ CSF exosomal RNA can be used to differentiate patients with Alzheimer's disease from control subjects with a reasonable degree of accuracy, given the relatively small number of samples used in this study. Studying the CSF RNA from a larger number of patients would help provide an insight into its potential as a disease biomarker. 

References 

1. Salas C, Broglio C, Rodriguez F: Evolution of forebrain and spatial cognition in vertebrates: conservation across diversity. Brain, behavior and evolution 2003, 62(2):72-82.

2. Lui JH, Hansen DV, Kriegstein AR: Development and evolution of the human neocortex. Cell 2011, 146(1):18-36.

3. Miller EK, Freedman DJ, Wallis JD: The prefrontal cortex: categories, concepts and cognition. Philosophical transactions of the Royal Society of London Series B, Biological sciences 2002, 357(1424):1123-1136.

4. Goldman-Rakic PS: Topography of cognition: parallel distributed networks in primate association cortex. Annual review of neuroscience 1988, 11:137-156.

5. Funahashi S: Prefrontal cortex and working memory processes. Neuroscience 2006, 139(1):251261.

6. Lebedev MA, Messinger A, Kralik JD, Wise SP: Representation of attended versus remembered locations in prefrontal cortex. PLoS biology 2004, 2(11):e365.

7. Bush G, Luu P, Posner Ml: Cognitive and emotional influences in anterior cingulate cortex. Trends in cognitive sciences 2000, 4(6):215-222.

8. Weible AP, Rowland DC, Monaghan CK, Wolfgang NT, Kentros CG: Neural correlates of long-term object memory in the mouse anterior cingulate cortex. The Journal of neuroscience : the official journal of the Society for Neuroscience 2012, 32(16):5598-5608.

9. Weible AP, Rowland DC, Pang R, Kentros C: Neural correlates of novel object and novel location recognition behavior in the mouse anterior cingulate cortex. Journal of neurophysiology 2009, 102(4):20552068.

10. Frankland PW, Bontempi B, Talton LE, Kaczmarek L, Silva AJ: The involvement of the anterior cingulate cortex in remote contextual fear memory. Science 2004, 304(5672):881-883.

11. Scoville WB, Milner B: Loss of recent memory after bilateral hippocampal lesions. Journal of neurology, neurosurgery, and psychiatry 1957, 20(1):11-21.

12. Moser E, Moser MB, Andersen P: Spatial learning impairment parallels the magnitude of dorsal hippocampal lesions, but is hardly present following ventral lesions. The Journal of neuroscience : the official journal of the Society for Neuroscience 1993, 13(9):3916-3925.

13. Mahut $\mathrm{H}$, Zola-Morgan S, Moss M: Hippocampal resections impair associative learning and recognition memory in the monkey. The Journal of neuroscience : the official journal of the Society for Neuroscience 1982, 2(9):1214-1220.

14. Sutherland RJ, O'Brien J, Lehmann H: Absence of systems consolidation of fear memories after dorsal, ventral, or complete hippocampal damage. Hippocampus 2008, 18(7):710-718.

15. Abbott LF, Nelson SB: Synaptic plasticity: taming the beast. Nature neuroscience 2000, 3 Suppl:1178-1183.

16. Martin SJ, Grimwood PD, Morris RG: Synaptic plasticity and memory: an evaluation of the hypothesis. Annual review of neuroscience 2000, 23:649-711.

17. Aggleton JP: A description of the amygdalo-hippocampal interconnections in the macaque monkey. Experimental brain research Experimentelle Hirnforschung Experimentation cerebrale 1986, 64(3):515526.

18. Green JD, Adey WR: Electrophysiological studies of hippocampal connections and excitability. Electroencephalography and clinical neurophysiology 1956, 8(2):245-263.

19. Laroche S, Davis S, Jay TM: Plasticity at hippocampal to prefrontal cortex synapses: dual roles in working memory and consolidation. Hippocampus 2000, 10(4):438-446.

20. Heath RG, Harper JW: Ascending projections of the cerebellar fastigial nucleus to the hippocampus, 
amygdala, and other temporal lobe sites: evoked potential and histological studies in monkeys and cats. Experimental neurology 1974, 45(2):268-287.

21. Vago DR, Kesner RP: Disruption of the direct perforant path input to the CA1 subregion of the dorsal hippocampus interferes with spatial working memory and novelty detection. Behavioural brain research 2008, 189(2):273-283.

22. Kumar A: Long-Term Potentiation at CA3-CA1 Hippocampal Synapses with Special Emphasis on Aging, Disease, and Stress. Frontiers in aging neuroscience 2011, 3:7.

23. Pothuizen HH, Zhang WN, Jongen-Relo AL, Feldon J, Yee BK: Dissociation of function between the dorsal and the ventral hippocampus in spatial learning abilities of the rat: a within-subject, withintask comparison of reference and working spatial memory. The European journal of neuroscience 2004, 19(3):705-712.

24. Fanselow MS, Dong HW: Are the dorsal and ventral hippocampus functionally distinct structures? Neuron 2010, 65(1):7-19.

25. Hyman BT, Van Hoesen GW, Damasio AR, Barnes CL: Alzheimer's disease: cell-specific pathology isolates the hippocampal formation. Science 1984, 225(4667):1168-1170.

26. Van Hoesen GW, Hyman BT: Hippocampal formation: anatomy and the patterns of pathology in Alzheimer's disease. Progress in brain research 1990, 83:445-457.

27. Hyman BT, Van Hoesen GW, Damasio AR: Memory-related neural systems in Alzheimer's disease: an anatomic study. Neurology 1990, 40(11):1721-1730.

28. Altman J: Autoradiographic investigation of cell proliferation in the brains of rats and cats. The Anatomical record 1963, 145:573-591.

29. Eriksson PS, Perfilieva E, Bjork-Eriksson T, Alborn AM, Nordborg C, Peterson DA, Gage FH: Neurogenesis in the adult human hippocampus. Nature medicine 1998, 4(11):1313-1317.

30. Deng W, Aimone JB, Gage FH: New neurons and new memories: how does adult hippocampal neurogenesis affect learning and memory? Nature reviews Neuroscience 2010, 11(5):339-350.

31. Marin-Burgin A, Schinder AF: Requirement of adult-born neurons for hippocampus-dependent learning. Behavioural brain research 2012, 227(2):391-399.

32. Hughes JR: Post-tetanic potentiation. Physiological reviews 1958, 38(1):91-113.

33. Douglas RM, Goddard GV: Long-term potentiation of the perforant path-granule cell synapse in the rat hippocampus. Brain research 1975, 86(2):205-215.

34. Bliss TV, Gardner-Medwin AR: Long-lasting potentiation of synaptic transmission in the dentate area of the unanaestetized rabbit following stimulation of the perforant path. The Journal of physiology 1973, 232(2):357-374.

35. Massey PV, Bashir ZI: Long-term depression: multiple forms and implications for brain function. Trends in neurosciences 2007, 30(4):176-184.

36. Abraham WC: How long will long-term potentiation last? Philosophical transactions of the Royal Society of London Series B, Biological sciences 2003, 358(1432):735-744.

37. Flexner JB, Flexner LB, Stellar E: Memory in mice as affected by intracerebral puromycin. Science 1963, 141(3575):57-59.

38. Frey U, Krug M, Reymann KG, Matthies H: Anisomycin, an inhibitor of protein synthesis, blocks late phases of LTP phenomena in the hippocampal CA1 region in vitro. Brain research 1988, 452(1-2):57-65.

39. Frey U, Frey S, Schollmeier F, Krug M: Influence of actinomycin D, a RNA synthesis inhibitor, on long-term potentiation in rat hippocampal neurons in vivo and in vitro. The Journal of physiology 1996, 490 ( Pt 3):703-711.

40. Lynch MA: Long-term potentiation and memory. Physiological reviews 2004, 84(1):87-136. 
41. Silva AJ, Kogan JH, Frankland PW, Kida S: CREB and memory. Annual review of neuroscience 1998, 21:127-148.

42. Frey U, Morris RG: Synaptic tagging and long-term potentiation. Nature 1997, 385(6616):533-536.

43. Bodian D: A Suggestive Relationship of Nerve Cell Rna with Specific Synaptic Sites. Proceedings of the National Academy of Sciences of the United States of America 1965, 53:418-425.

44. Sutton MA, Schuman EM: Dendritic protein synthesis, synaptic plasticity, and memory. Cell 2006, 127(1):49-58.

45. Kang $\mathrm{H}$, Schuman EM: A requirement for local protein synthesis in neurotrophin-induced hippocampal synaptic plasticity. Science 1996, 273(5280):1402-1406.

46. Milanesi G, Brody EN, Grau O, Geiduschek EP: Transcriptions of the bacteriophage T4 template in vitro: separation of "delayed early" from "immediate early" transcription. Proceedings of the National Academy of Sciences of the United States of America 1970, 66(1):181-188.

47. Chen CY, Forman LW, Faller DV: Calcium-dependent immediate-early gene induction in lymphocytes is negatively regulated by p21Ha-ras. Molecular and cellular biology 1996, 16(11):6582-6592.

48. Saadane N, Alpert L, Chalifour LE: Expression of immediate early genes, GATA-4, and Nkx-2.5 in adrenergic-induced cardiac hypertrophy and during regression in adult mice. British journal of pharmacology 1999, 127(5):1165-1176.

49. Cole AJ, Saffen DW, Baraban JM, Worley PF: Rapid increase of an immediate early gene messenger RNA in hippocampal neurons by synaptic NMDA receptor activation. Nature 1989, 340(6233):474-476.

50. Qian Z, Gilbert ME, Colicos MA, Kandel ER, Kuhl D: Tissue-plasminogen activator is induced as an immediate-early gene during seizure, kindling and long-term potentiation. Nature 1993, 361(6411):453457.

51. Bramham CR, Worley PF, Moore MJ, Guzowski JF: The immediate early gene arc/arg3.1: regulation, mechanisms, and function. The Journal of neuroscience : the official journal of the Society for Neuroscience 2008, 28(46):11760-11767.

52. Jones MW, Errington ML, French PJ, Fine A, Bliss TV, Garel S, Charnay P, Bozon B, Laroche S, Davis S: A requirement for the immediate early gene Zif268 in the expression of late LTP and long-term memories. Nature neuroscience 2001, 4(3):289-296.

53. Tischmeyer W, Grimm R: Activation of immediate early genes and memory formation. Cellular and molecular life sciences : CMLS 1999, 55(4):564-574.

54. von Hertzen LS, Giese KP: Memory reconsolidation engages only a subset of immediate-early genes induced during consolidation. The Journal of neuroscience : the official journal of the Society for Neuroscience 2005, 25(8):1935-1942.

55. Impey S, Smith DM, Obrietan K, Donahue R, Wade C, Storm DR: Stimulation of cAMP response element (CRE)-mediated transcription during contextual learning. Nature neuroscience 1998, 1(7):595601.

56. Xu X, Zhan M, Duan W, Prabhu V, Brenneman R, Wood W, Firman J, Li H, Zhang P, Ibe C et al: Gene expression atlas of the mouse central nervous system: impact and interactions of age, energy intake and gender. Genome biology 2007, 8(11):R234.

57. Berchtold NC, Cribbs DH, Coleman PD, Rogers J, Head E, Kim R, Beach T, Miller C, Troncoso J, Trojanowski JQ et al: Gene expression changes in the course of normal brain aging are sexually dimorphic. Proceedings of the National Academy of Sciences of the United States of America 2008, 105(40):1560515610.

58. Lu T, Pan Y, Kao SY, Li C, Kohane I, Chan J, Yankner BA: Gene regulation and DNA damage in the ageing human brain. Nature 2004, 429(6994):883-891. 
59. Erraji-Benchekroun L, Underwood MD, Arango V, Galfalvy H, Pavlidis P, Smyrniotopoulos P, Mann $\mathrm{JJ}$, Sibille E: Molecular aging in human prefrontal cortex is selective and continuous throughout adult life. Biological psychiatry 2005, 57(5):549-558.

60. Glorioso C, Oh S, Douillard GG, Sibille E: Brain molecular aging, promotion of neurological disease and modulation by sirtuin 5 longevity gene polymorphism. Neurobiology of disease 2011, 41(2):279-290.

61. Avramopoulos D, Szymanski M, Wang R, Bassett S: Gene expression reveals overlap between normal aging and Alzheimer's disease genes. Neurobiology of aging 2011, 32(12):2319 e2327-2334.

62. Miller JA, Oldham MC, Geschwind DH: A systems level analysis of transcriptional changes in Alzheimer's disease and normal aging. The Journal of neuroscience : the official journal of the Society for Neuroscience 2008, 28(6):1410-1420.

63. Peleg S, Sananbenesi F, Zovoilis A, Burkhardt S, Bahari-Javan S, Agis-Balboa RC, Cota P, Wittnam $\mathrm{JL}$, Gogol-Doering A, Opitz $\mathrm{L}$ et al: Altered histone acetylation is associated with age-dependent memory impairment in mice. Science 2010, 328(5979):753-756.

64. Burger C, Lopez MC, Baker HV, Mandel RJ, Muzyczka N: Genome-wide analysis of aging and learning-related genes in the hippocampal dentate gyrus. Neurobiology of learning and memory 2008 , 89(4):379-396.

65. Jing Q, Huang S, Guth S, Zarubin T, Motoyama A, Chen J, Di Padova F, Lin SC, Gram H, Han J: Involvement of microRNA in AU-rich element-mediated mRNA instability. Cell 2005, 120(5):623-634.

66. Lee RC, Feinbaum RL, Ambros V: The C. elegans heterochronic gene lin-4 encodes small RNAs with antisense complementarity to lin-14. Cell 1993, 75(5):843-854.

67. Wienholds E, Koudijs MJ, van Eeden FJ, Cuppen E, Plasterk RH: The microRNA-producing enzyme Dicer1 is essential for zebrafish development. Nature genetics 2003, 35(3):217-218.

68. de Pontual L, Yao E, Callier P, Faivre L, Drouin V, Cariou S, Van Haeringen A, Genevieve D, Goldenberg A, Oufadem $M$ et al: Germline deletion of the miR-17 approximately 92 cluster causes skeletal and growth defects in humans. Nature genetics 2011, 43(10):1026-1030.

69. Esquela-Kerscher A, Slack FJ: Oncomirs - microRNAs with a role in cancer. Nature reviews Cancer 2006, 6(4):259-269.

70. Schratt G: microRNAs at the synapse. Nature reviews Neuroscience 2009, 10(12):842-849.

71. Kozomara A, Griffiths-Jones S: miRBase: integrating microRNA annotation and deep-sequencing data. Nucleic acids research 2011, 39(Database issue):D152-157.

72. Rajasethupathy P, Fiumara F, Sheridan R, Betel D, Puthanveettil SV, Russo JJ, Sander C, Tuschl T, Kandel E: Characterization of small RNAs in Aplysia reveals a role for miR-124 in constraining synaptic plasticity through CREB. Neuron 2009, 63(6):803-817.

73. Gao J, Wang WY, Mao YW, Graff J, Guan JS, Pan L, Mak G, Kim D, Su SC, Tsai LH: A novel pathway regulates memory and plasticity via SIRT1 and miR-134. Nature 2010, 466(7310):1105-1109.

74. Pigazzi M, Manara E, Baron E, Basso G: miR-34b targets cyclic AMP-responsive element binding protein in acute myeloid leukemia. Cancer research 2009, 69(6):2471-2478.

75. Wayman GA, Davare M, Ando H, Fortin D, Varlamova O, Cheng HY, Marks D, Obrietan K, Soderling TR, Goodman RH et al: An activity-regulated microRNA controls dendritic plasticity by downregulating p250GAP. Proceedings of the National Academy of Sciences of the United States of America 2008, 105(26):9093-9098.

76. Impey S, Davare M, Lesiak A, Fortin D, Ando H, Varlamova O, Obrietan K, Soderling TR, Goodman $\mathrm{RH}$, Wayman GA: An activity-induced microRNA controls dendritic spine formation by regulating Rac1-PAK signaling. Molecular and cellular neurosciences 2010, 43(1):146-156.

77. Siegel G, Obernosterer G, Fiore R, Oehmen M, Bicker S, Christensen M, Khudayberdiev S, 
Leuschner PF, Busch CJ, Kane C et al: A functional screen implicates microRNA-138-dependent regulation of the depalmitoylation enzyme APT1 in dendritic spine morphogenesis. Nature cell biology 2009, 11(6):705716.

78. Wu J, Xie X: Comparative sequence analysis reveals an intricate network among REST, CREB and miRNA in mediating neuronal gene expression. Genome biology 2006, 7(9):R85.

79. Laneve P, Gioia U, Andriotto A, Moretti F, Bozzoni I, Caffarelli E: A minicircuitry involving REST and CREB controls miR-9-2 expression during human neuronal differentiation. Nucleic acids research 2010, 38(20):6895-6905.

80. Kim J, Krichevsky A, Grad Y, Hayes GD, Kosik KS, Church GM, Ruvkun G: Identification of many microRNAs that copurify with polyribosomes in mammalian neurons. Proceedings of the National Academy of Sciences of the United States of America 2004, 101(1):360-365.

81. Barbee SA, Estes PS, Cziko AM, Hillebrand J, Luedeman RA, Coller JM, Johnson N, Howlett IC, Geng C, Ueda R et al: Staufen- and FMRP-containing neuronal RNPs are structurally and functionally related to somatic $P$ bodies. Neuron 2006, 52(6):997-1009.

82. Lugli G, Larson J, Martone ME, Jones Y, Smalheiser NR: Dicer and elF2c are enriched at postsynaptic densities in adult mouse brain and are modified by neuronal activity in a calpain-dependent manner. Journal of neurochemistry 2005, 94(4):896-905.

83. Zhang Y, Ueno Y, Liu XS, Buller B, Wang X, Chopp M, Zhang ZG: The MicroRNA-17-92 cluster enhances axonal outgrowth in embryonic cortical neurons. The Journal of neuroscience : the official journal of the Society for Neuroscience 2013, 33(16):6885-6894.

84. Dajas-Bailador F, Bonev B, Garcez P, Stanley P, Guillemot F, Papalopulu N: microRNA-9 regulates axon extension and branching by targeting Map1b in mouse cortical neurons. Nature neuroscience 2012.

85. Zovoilis A, Agbemenyah HY, Agis-Balboa RC, Stilling RM, Edbauer D, Rao P, Farinelli L, Delalle I, Schmitt A, Falkai $P$ et al: microRNA-34c is a novel target to treat dementias. The EMBO journal 2011, 30(20):4299-4308.

86. Schipper HM, Maes OC, Chertkow HM, Wang E: MicroRNA expression in Alzheimer blood mononuclear cells. Gene regulation and systems biology 2007, 1:263-274.

87. Li X, Khanna A, Li N, Wang E: Circulatory miR34a as an RNAbased, noninvasive biomarker for brain aging. Aging 2011, 3(10):985-1002.

88. Khanna A, Muthusamy S, Liang R, Sarojini H, Wang E: Gain of survival signaling by down-regulation of three key miRNAs in brain of calorie-restricted mice. Aging 2011, 3(3):223-236.

89. Liu N, Landreh M, Cao K, Abe M, Hendriks GJ, Kennerdell JR, Zhu Y, Wang LS, Bonini NM: The microRNA miR-34 modulates ageing and neurodegeneration in Drosophila. Nature 2012, 482(7386):519523.

90. Bernstein E, Kim SY, Carmell MA, Murchison EP, Alcorn H, Li MZ, Mills AA, Elledge SJ, Anderson KV, Hannon GJ: Dicer is essential for mouse development. Nature genetics 2003, 35(3):215-217.

91. Kanellopoulou C, Muljo SA, Kung AL, Ganesan S, Drapkin R, Jenuwein T, Livingston DM, Rajewsky $\mathrm{K}$ : Dicer-deficient mouse embryonic stem cells are defective in differentiation and centromeric silencing. Genes \& development 2005, 19(4):489-501.

92. Miska EA, Alvarez-Saavedra E, Abbott AL, Lau NC, Hellman AB, McGonagle SM, Bartel DP, Ambros VR, Horvitz HR: Most Caenorhabditis elegans microRNAs are individually not essential for development or viability. PLoS genetics 2007, 3(12):e215.

93. Remenyi J, van den Bosch MW, Palygin O, Mistry RB, McKenzie C, Macdonald A, Hutvagner G, Arthur JS, Frenguelli BG, Pankratov Y: miR-132/212 knockout mice reveal roles for these miRNAs in regulating cortical synaptic transmission and plasticity. PloS one 2013, 8(4):e62509. 
94. van Rooij E, Quiat D, Johnson BA, Sutherland LB, Qi X, Richardson JA, Kelm RJ, Jr., Olson EN: A family of microRNAs encoded by myosin genes governs myosin expression and muscle performance. Developmental cell 2009, 17(5):662-673.

95. Concepcion CP, Han YC, Mu P, Bonetti C, Yao E, D'Andrea A, Vidigal JA, Maughan WP, Ogrodowski $P$, Ventura A: Intact p53-dependent responses in miR-34-deficient mice. PLoS genetics 2012, 8(7):e1002797.

96. Wu Cl, Shen $\mathrm{Y}$, Tang T: Evolution under canalization and the dual roles of microRNAs: a hypothesis. Genome research 2009, 19(5):734-743.

97. Baek D, Villen J, Shin C, Camargo FD, Gygi SP, Bartel DP: The impact of microRNAs on protein output. Nature 2008, 455(7209):64-71.

98. Selbach M, Schwanhausser B, Thierfelder N, Fang Z, Khanin R, Rajewsky N: Widespread changes in protein synthesis induced by microRNAs. Nature 2008, 455(7209):58-63.

99. Yang Z, Dong D, Zhang Z, Crabbe MJ, Wang L, Zhong Y: Preferential regulation of stably expressed genes in the human genome suggests a widespread expression buffering role of microRNAs. BMC genomics 2012, 13 Suppl 7:S14.

100. Morris R: Developments of a water-maze procedure for studying spatial learning in the rat. Journal of neuroscience methods 1984, 11(1):47-60.

101. Morris RG, Garrud P, Rawlins JN, O'Keefe J: Place navigation impaired in rats with hippocampal lesions. Nature 1982, 297(5868):681-683.

102. LeDoux JE: Emotion circuits in the brain. Annual review of neuroscience 2000, 23:155-184.

103. Phillips RG, LeDoux JE: Differential contribution of amygdala and hippocampus to cued and contextual fear conditioning. Behavioral neuroscience 1992, 106(2):274-285.

104. Antunes M, Biala G: The novel object recognition memory: neurobiology, test procedure, and its modifications. Cognitive processing 2012, 13(2):93-110.

105. Martinez M, HernandezAl, Martinez N: N-Acetylcysteine delays age-associated memory impairment in mice: role in synaptic mitochondria. Brain research 2000, 855(1):100-106.

106. Flood JF, Roberts E: Dehydroepiandrosterone sulfate improves memory in aging mice. Brain research 1988, 448(1):178-181.

107. Lander ES, Linton LM, Birren B, Nusbaum C, Zody MC, Baldwin J, Devon K, Dewar K, Doyle M, FitzHugh W et al: Initial sequencing and analysis of the human genome. Nature 2001, 409(6822):860-921. 108. Alwine JC, Kemp DJ, Stark GR: Method for detection of specific RNAs in agarose gels by transfer to diazobenzyloxymethyl-paper and hybridization with DNA probes. Proceedings of the National Academy of Sciences of the United States of America 1977, 74(12):5350-5354.

109. Streit S, Michalski CW, Erkan M, Kleeff J, Friess H: Northern blot analysis for detection and quantification of RNA in pancreatic cancer cells and tissues. Nature protocols 2009, 4(1):37-43.

110. Brunner AM, Yakovlev IA, Strauss SH: Validating internal controls for quantitative plant gene expression studies. BMC plant biology 2004, 4:14.

111. Dittmer DP: Transcription profile of Kaposi's sarcoma-associated herpesvirus in primary Kaposi's sarcoma lesions as determined by real-time PCR arrays. Cancer research 2003, 63(9):2010-2015.

112. Juan D, Alexe G, Antes T, Liu H, Madabhushi A, Delisi C, Ganesan S, Bhanot G, Liou LS: Identification of a microRNA panel for clear-cell kidney cancer. Urology 2010, 75(4):835-841.

113. Hurd PJ, Nelson CJ: Advantages of next-generation sequencing versus the microarray in epigenetic research. Briefings in functional genomics \& proteomics 2009, 8(3):174-183.

114. ten Bosch JR, Grody WW: Keeping up with the next generation: massively parallel sequencing in clinical diagnostics. The Journal of molecular diagnostics : JMD 2008, 10(6):484-492. 
115. Poliseno L, Salmena L, Zhang J, Carver B, Haveman WJ, Pandolfi PP: A coding-independent function of gene and pseudogene mRNAs regulates tumour biology. Nature 2010, 465(7301):1033-1038.

116. Memczak S, Jens M, Elefsinioti A, Torti F, Krueger J, Rybak A, Maier L, Mackowiak SD, Gregersen $\mathrm{LH}$, Munschauer $\mathrm{M}$ et al: Circular RNAs are a large class of animal RNAs with regulatory potency. Nature 2013, 495(7441):333-338.

117. Landfield PW: Hippocampal neurobiological mechanisms of age-related memory dysfunction. Neurobiology of aging 1988, 9(5-6):571-579.

118. Francis PT, Palmer AM, Snape M, Wilcock GK: The cholinergic hypothesis of Alzheimer's disease: a review of progress. Journal of neurology, neurosurgery, and psychiatry 1999, 66(2):137-147.

119. Drugs for Alzheimer's disease: best avoided. No therapeutic advantage. Prescrire international 2012, 21(128): 150.

120. Goate A, Chartier-Harlin MC, Mullan M, Brown J, Crawford F, Fidani L, Giuffra L, Haynes A, Irving $\mathrm{N}$, James $\mathrm{L}$ et al: Segregation of a missense mutation in the amyloid precursor protein gene with familial Alzheimer's disease. Nature 1991, 349(6311):704-706.

121. Sherrington R, Rogaev El, Liang Y, Rogaeva EA, Levesque G, Ikeda M, Chi H, Lin C, Li G, Holman $\mathrm{K}$ et al: Cloning of a gene bearing missense mutations in early-onset familial Alzheimer's disease. Nature 1995, 375(6534):754-760.

122. Goedert M, Spillantini MG, Crowther RA: Tau proteins and neurofibrillary degeneration. Brain Pathol 1991, 1(4):279-286.

123. Rizzu P, Van Swieten JC, Joosse M, Hasegawa M, Stevens M, Tibben A, Niermeijer MF, Hillebrand M, Ravid R, Oostra BA et al: High prevalence of mutations in the microtubule-associated protein tau in a population study of frontotemporal dementia in the Netherlands. American journal of human genetics 1999 , 64(2):414-421.

124. Roberson ED, Halabisky B, Yoo JW, Yao J, Chin J, Yan F, Wu T, Hamto P, Devidze N, Yu GQ et al: Amyloid-beta/Fyn-induced synaptic, network, and cognitive impairments depend on tau levels in multiple mouse models of Alzheimer's disease. The Journal of neuroscience : the official journal of the Society for Neuroscience 2011, 31(2):700-711.

125. Kane MD, Lipinski WJ, Callahan MJ, Bian F, Durham RA, Schwarz RD, Roher AE, Walker LC: Evidence for seeding of beta -amyloid by intracerebral infusion of Alzheimer brain extracts in beta -amyloid precursor protein-transgenic mice. The Journal of neuroscience : the official journal of the Society for Neuroscience 2000, 20(10):3606-3611.

126. Eisele YS, Obermuller U, Heilbronner G, Baumann F, Kaeser SA, Wolburg H, Walker LC, Staufenbiel M, Heikenwalder M, Jucker M: Peripherally applied Abeta-containing inoculates induce cerebral betaamyloidosis. Science 2010, 330(6006):980-982.

127. Liu L, Drouet V, Wu JW, Witter MP, Small SA, Clelland C, Duff K: Trans-synaptic spread of tau pathology in vivo. PloS one 2012, 7(2):e31302.

128. de Calignon A, Polydoro M, Suarez-Calvet M, William C, Adamowicz DH, Kopeikina KJ, Pitstick R, Sahara N, Ashe KH, Carlson GA et al: Propagation of tau pathology in a model of early Alzheimer's disease. Neuron 2012, 73(4):685-697.

129. Vingtdeux V, Sergeant N, Buee L: Potential contribution of exosomes to the prion-like propagation of lesions in Alzheimer's disease. Frontiers in physiology 2012, 3:229.

130. Aguzzi A, Rajendran L: The transcellular spread of cytosolic amyloids, prions, and prionoids. Neuron 2009, 64(6):783-790.

131. Costanzo M, Zurzolo C: The cell biology of prion-like spread of protein aggregates: mechanisms and implication in neurodegeneration. The Biochemical journal 2013, 452(1):1-17. 
132. Evans DA, Funkenstein HH, Albert MS, Scherr PA, Cook NR, Chown MJ, Hebert LE, Hennekens $\mathrm{CH}$, Taylor JO: Prevalence of Alzheimer's disease in a community population of older persons. Higher than previously reported. JAMA : the journal of the American Medical Association 1989, 262(18):2551-2556.

133. Markesbery WR: Oxidative stress hypothesis in Alzheimer's disease. Free radical biology \& medicine 1997, 23(1):134-147.

134. Squitti R: Metals in Alzheimer's disease: a systemic perspective. Front Biosci (Landmark Ed) 2012, 17:451-472.

135. Singh I, Sagare AP, Coma M, Perlmutter D, Gelein R, Bell RD, Deane RJ, Zhong E, Parisi M, Ciszewski $J$ et al: Low levels of copper disrupt brain amyloid-beta homeostasis by altering its production and clearance. Proceedings of the National Academy of Sciences of the United States of America 2013, 110(36):14771-14776.

136. Palmer AM, Procter AW, Stratmann GC, Bowen DM: Excitatory amino acid-releasing and cholinergic neurones in Alzheimer's disease. Neuroscience letters 1986, 66(2):199-204.

137. Gureje O, Ogunniyi A, Baiyewu O, Price B, Unverzagt FW, Evans RM, Smith-Gamble V, Lane KA, Gao S, Hall KS et al: APOE epsilon4 is not associated with Alzheimer's disease in elderly Nigerians. Annals of neurology 2006, 59(1):182-185.

138. Osuntokun BO, Sahota A, Ogunniyi AO, Gureje O, Baiyewu O, Adeyinka A, Oluwole SO, Komolafe $\mathrm{O}$, Hall KS, Unverzagt FW et al: Lack of an association between apolipoprotein E epsilon 4 and Alzheimer's disease in elderly Nigerians. Annals of neurology 1995, 38(3):463-465.

139. Doody RS, Pavlik V, Massman P, Rountree S, Darby E, Chan W: Predicting progression of Alzheimer's disease. Alzheimer's research \& therapy 2010, 2(1):2.

140. Dawson SJ, Rueda OM, Aparicio S, Caldas C: A new genome-driven integrated classification of breast cancer and its implications. The EMBO journal 2013, 32(5):617-628.

141. Pericak-Vance MA, Bebout JL, Gaskell PC, Jr., Yamaoka LH, Hung WY, Alberts MJ, Walker AP, Bartlett RJ, Haynes CA, Welsh KA et al: Linkage studies in familial Alzheimer disease: evidence for chromosome 19 linkage. American journal of human genetics 1991, 48(6):1034-1050.

142. Backman L, Jones S, Berger AK, Laukka EJ, Small BJ: Multiple cognitive deficits during the transition to Alzheimer's disease. Journal of internal medicine 2004, 256(3):195-204.

143. Mudher A, Lovestone S: Alzheimer's disease-do tauists and baptists finally shake hands? Trends in neurosciences 2002, 25(1):22-26.

144. Hebert LE, Scherr PA, Beckett LA, Albert MS, Pilgrim DM, Chown MJ, Funkenstein HH, Evans DA: Age-specific incidence of Alzheimer's disease in a community population. JAMA : the journal of the American Medical Association 1995, 273(17):1354-1359.

145. Buckner RL: Memory and executive function in aging and AD: multiple factors that cause decline and reserve factors that compensate. Neuron 2004, 44(1):195-208.

146. Koivisto K, Reinikainen KJ, Hanninen T, Vanhanen M, Helkala EL, Mykkanen L, Laakso M, Pyorala K, Riekkinen PJ, Sr.: Prevalence of age-associated memory impairment in a randomly selected population from eastern Finland. Neurology 1995, 45(4):741-747.

147. Hanninen T, Hallikainen M, Koivisto K, Helkala EL, Reinikainen KJ, Soininen H, Mykkanen L, Laakso M, Pyorala K, Riekkinen PJ, Sr.: A follow-up study of age-associated memory impairment: neuropsychological predictors of dementia. Journal of the American Geriatrics Society 1995, 43(9):10071015.

148. West MJ, Coleman PD, Flood DG, Troncoso JC: Differences in the pattern of hippocampal neuronal loss in normal ageing and Alzheimer's disease. Lancet 1994, 344(8925):769-772. 
149. Small SA, Schobel SA, Buxton RB, Witter MP, Barnes CA: A pathophysiological framework of hippocampal dysfunction in ageing and disease. Nature reviews Neuroscience 2011, 12(10):585-601.

150. Wang J, Dickson DW, Trojanowski JQ, Lee VM: The levels of soluble versus insoluble brain Abeta distinguish Alzheimer's disease from normal and pathologic aging. Experimental neurology 1999, 158(2):328-337.

151. Forstl H, Hentschel F, Sattel H, Geiger-Kabisch C, Besthorn C, Czech C, Monning U, Beyreuther $\mathrm{K}$ : Age-associated memory impairment and early Alzheimer's disease. Only time will tell the difference. Arzneimittel-Forschung 1995, 45(3A):394-397.

152. Caselli RJ, Dueck AC, Osborne D, Sabbagh MN, Connor DJ, Ahern GL, Baxter LC, Rapcsak SZ, Shi J, Woodruff BK et al: Longitudinal modeling of age-related memory decline and the APOE epsilon4 effect. The New England journal of medicine 2009, 361(3):255-263.

153. Palumbo B, Parnetti L, Nocentini G, Cardinali L, Brancorsini S, Riccardi C, Senin U: Apolipoprotein-E genotype in normal aging, age-associated memory impairment, Alzheimer's disease and vascular dementia patients. Neuroscience letters 1997, 231(1):59-61.

154. Bartres-Faz D, Junque C, Lopez-Alomar A, Valveny N, Moral P, Casamayor R, Salido A, Bel C, Clemente IC: Neuropsychological and genetic differences between age-associated memory impairment and mild cognitive impairment entities. Journal of the American Geriatrics Society 2001, 49(7):985-990.

155. Morrison JH, Hof PR: Life and death of neurons in the aging brain. Science 1997, 278(5337):412419.

156. Morrison JH, Hof PR: Selective vulnerability of corticocortical and hippocampal circuits in aging and Alzheimer's disease. Progress in brain research 2002, 136:467-486.

157. Gazzaley A, Cooney JW, Rissman J, D’Esposito M: Top-down suppression deficit underlies working memory impairment in normal aging. Nature neuroscience 2005, 8(10):1298-1300.

158. Miller SL, Celone K, DePeau K, Diamond E, Dickerson BC, Rentz D, Pihlajamaki M, Sperling RA: Age-related memory impairment associated with loss of parietal deactivation but preserved hippocampal activation. Proceedings of the National Academy of Sciences of the United States of America 2008, 105(6):2181-2186.

159. Gazzaley A, D'Esposito M: Top-down modulation and normal aging. Annals of the New York Academy of Sciences 2007, 1097:67-83.

160. Pavlopoulos E, Jones S, Kosmidis S, Close M, Kim C, Kovalerchik O, Small SA, Kandel ER: Molecular mechanism for age-related memory loss: the histone-binding protein RbAp48. Science translational medicine 2013, 5(200):200ra115.

161. Butterfield DA, Howard B, Yatin S, Koppal T, Drake J, Hensley K, Aksenov M, Aksenova M, Subramaniam R, Varadarajan $S$ et al: Elevated oxidative stress in models of normal brain aging and Alzheimer's disease. Life sciences 1999, 65(18-19):1883-1892.

162. Perrin RJ, Fagan AM, Holtzman DM: Multimodal techniques for diagnosis and prognosis of Alzheimer's disease. Nature 2009, 461(7266):916-922.

163. Jack CR, Jr., Knopman DS, Jagust WJ, Petersen RC, Weiner MW, Aisen PS, Shaw LM, Vemuri P, Wiste HJ, Weigand SD et al: Tracking pathophysiological processes in Alzheimer's disease: an updated hypothetical model of dynamic biomarkers. Lancet neurology 2013, 12(2):207-216.

164. Hock C, Golombowski S, Muller-Spahn F, Naser W, Beyreuther K, Monning U, Schenk D, VigoPelfrey C, Bush AM, Moir R et al: Cerebrospinal fluid levels of amyloid precursor protein and amyloid betapeptide in Alzheimer's disease and major depression - inverse correlation with dementia severity. European neurology 1998, 39(2):111-118.

165. De Meyer G, Shapiro F, Vanderstichele H, Vanmechelen E, Engelborghs S, De Deyn PP, Coart E, 
Hansson $\mathrm{O}$, Minthon L, Zetterberg $\mathrm{H}$ et al: Diagnosis-independent Alzheimer disease biomarker signature in cognitively normal elderly people. Archives of neurology 2010, 67(8):949-956.

166. Galasko D, Chang L, Motter R, Clark CM, Kaye J, Knopman D, Thomas R, Kholodenko D, Schenk D, Lieberburg I et al: High cerebrospinal fluid tau and low amyloid beta42 levels in the clinical diagnosis of Alzheimer disease and relation to apolipoprotein E genotype. Archives of neurology 1998, 55(7):937-945.

167. Marksteiner J, Hinterhuber H, Humpel C: Cerebrospinal fluid biomarkers for diagnosis of Alzheimer's disease: beta-amyloid(1-42), tau, phospho-tau-181 and total protein. Drugs Today (Barc) 2007, 43(6):423431.

168. Mehta PD, Pirttila T, Mehta SP, Sersen EA, Aisen PS, Wisniewski HM: Plasma and cerebrospinal fluid levels of amyloid beta proteins 1-40 and 1-42 in Alzheimer disease. Archives of neurology 2000, 57(1):100-105.

169. Andreasen N, Minthon L, Clarberg A, Davidsson P, Gottfries J, Vanmechelen E, Vanderstichele H, Winblad B, Blennow K: Sensitivity, specificity, and stability of CSF-tau in AD in a community-based patient sample. Neurology 1999, 53(7):1488-1494.

170. Andreasen N, Vanmechelen E, Van de Voorde A, Davidsson P, Hesse C, Tarvonen S, Raiha I, Sourander L, Winblad B, Blennow K: Cerebrospinal fluid tau protein as a biochemical marker for Alzheimer's disease: a community based follow up study. Journal of neurology, neurosurgery, and psychiatry 1998, 64(3):298-305.

171. Verwey NA, van der Flier WM, Blennow K, Clark C, Sokolow S, De Deyn PP, Galasko D, Hampel H, Hartmann T, Kapaki E et al: A worldwide multicentre comparison of assays for cerebrospinal fluid biomarkers in Alzheimer's disease. Annals of clinical biochemistry 2009, 46(Pt 3):235-240.

172. Bibl M, Esselmann H, Otto M, Lewczuk P, Cepek L, Ruther E, Kornhuber J, Wiltfang J: Cerebrospinal fluid amyloid beta peptide patterns in Alzheimer's disease patients and nondemented controls depend on sample pretreatment: indication of carrier-mediated epitope masking of amyloid beta peptides. Electrophoresis 2004, 25(17):2912-2918.

173. Bjerke M, Portelius E, Minthon L, Wallin A, Anckarsater H, Anckarsater R, Andreasen N, Zetterberg $\mathrm{H}$, Andreasson $\mathrm{U}$, Blennow $\mathrm{K}$ : Confounding factors influencing amyloid Beta concentration in cerebrospinal fluid. International journal of Alzheimer's disease 2010, 2010.

174. Dubois B, Feldman HH, Jacova C, Dekosky ST, Barberger-Gateau P, Cummings J, Delacourte A, Galasko D, Gauthier S, Jicha $G$ et al: Research criteria for the diagnosis of Alzheimer's disease: revising the NINCDS-ADRDA criteria. Lancet neurology 2007, 6(8):734-746.

175. Jack CR, Jr., Petersen RC, Xu YC, O'Brien PC, Smith GE, Ivnik RJ, Boeve BF, Waring SC, Tangalos EG, Kokmen E: Prediction of AD with MRI-based hippocampal volume in mild cognitive impairment. Neurology 1999, 52(7):1397-1403.

176. de Souza LC, Chupin M, Bertoux M, Lehericy S, Dubois B, Lamari F, Le Ber I, Bottlaender M, Colliot O, Sarazin M: Is hippocampal volume a good marker to differentiate Alzheimer's disease from frontotemporal dementia? Journal of Alzheimer's disease : JAD 2013, 36(1):57-66.

177. Carpenter AP, Jr., Pontecorvo MJ, Hefti FF, Skovronsky DM: The use of the exploratory IND in the evaluation and development of 18F-PET radiopharmaceuticals for amyloid imaging in the brain: a review of one company's experience. Q J Nucl Med Mol Imaging 2009, 53(4):387-393.

178. Dougall NJ, Bruggink S, Ebmeier KP: Systematic review of the diagnostic accuracy of 99mTcHMPAO-SPECT in dementia. The American journal of geriatric psychiatry : official journal of the American Association for Geriatric Psychiatry 2004, 12(6):554-570.

179. Koronyo-Hamaoui M, Koronyo Y, Ljubimov AV, Miller CA, Ko MK, Black KL, Schwartz M, Farkas DL: Identification of amyloid plaques in retinas from Alzheimer's patients and noninvasive in vivo optical 
imaging of retinal plaques in a mouse model. Neurolmage 2011, 54 Suppl 1:S204-217.

180. Wesson DW, Wilson DA, Nixon RA: Should olfactory dysfunction be used as a biomarker of Alzheimer's disease? Expert review of neurotherapeutics 2010, 10(5):633-635.

181. Trushina E, Dutta T, Persson XM, Mielke MM, Petersen RC: Identification of altered metabolic pathways in plasma and CSF in mild cognitive impairment and Alzheimer's disease using metabolomics. PloS one 2013, 8(5):e63644.

182. Valadi H, Ekstrom K, Bossios A, Sjostrand M, Lee JJ, Lotvall JO: Exosome-mediated transfer of mRNAs and microRNAs is a novel mechanism of genetic exchange between cells. Nature cell biology 2007, 9(6):654-659.

183. Kogure T, Lin WL, Yan IK, Braconi C, Patel T: Intercellular nanovesicle-mediated microRNA transfer: a mechanism of environmental modulation of hepatocellular cancer cell growth. Hepatology 2011, 54(4):1237-1248.

184. Montecalvo A, Larregina AT, Shufesky WJ, Stolz DB, Sullivan ML, Karlsson JM, Baty CJ, Gibson GA, Erdos G, Wang Z et al: Mechanism of transfer of functional microRNAs between mouse dendritic cells via exosomes. Blood 2012, 119(3):756-766.

185. Skog J, Wurdinger T, van Rijn S, Meijer DH, Gainche L, Sena-Esteves M, Curry WT, Jr., Carter BS, Krichevsky AM, Breakefield XO: Glioblastoma microvesicles transport RNA and proteins that promote tumour growth and provide diagnostic biomarkers. Nature cell biology 2008, 10(12):1470-1476.

186. Peinado H, Aleckovic M, Lavotshkin S, Matei I, Costa-Silva B, Moreno-Bueno G, Hergueta-Redondo M, Williams C, Garcia-Santos G, Ghajar C et al: Melanoma exosomes educate bone marrow progenitor cells toward a pro-metastatic phenotype through MET. Nature medicine 2012, 18(6):883-891.

187. Zhang Y, Liu D, Chen X, Li J, Li L, Bian Z, Sun F, Lu J, Yin Y, Cai X et al: Secreted monocytic miR150 enhances targeted endothelial cell migration. Molecular cell 2010, 39(1):133-144.

188. Yuan A, Farber EL, Rapoport AL, Tejada D, Deniskin R, Akhmedov NB, Farber DB: Transfer of microRNAs by embryonic stem cell microvesicles. PloS one 2009, 4(3):e4722.

189. Roberts CT, Jr., Kurre P: Vesicle trafficking and RNA transfer add complexity and connectivity to cell-cell communication. Cancer research 2013, 73(11):3200-3205.

190. Ratajczak J, Wysoczynski M, Hayek F, Janowska-Wieczorek A, Ratajczak MZ: Membrane-derived microvesicles: important and underappreciated mediators of cell-to-cell communication. Leukemia 2006, 20(9):1487-1495.

191. Faure J, Lachenal G, Court M, Hirrlinger J, Chatellard-Causse C, Blot B, Grange J, Schoehn G, Goldberg Y, Boyer $V$ et al: Exosomes are released by cultured cortical neurones. Molecular and cellular neurosciences 2006, 31(4):642-648.

192. Lachenal G, Pernet-Gallay K, Chivet M, Hemming FJ, Belly A, Bodon G, Blot B, Haase G, Goldberg $\mathrm{Y}$, Sadoul R: Release of exosomes from differentiated neurons and its regulation by synaptic glutamatergic activity. Molecular and cellular neurosciences 2011, 46(2):409-418.

193. Bakhti M, Winter C, Simons M: Inhibition of myelin membrane sheath formation by oligodendrocytederived exosome-like vesicles. The Journal of biological chemistry 2011, 286(1):787-796.

194. Fruhbeis C, Frohlich D, Kuo WP, Amphornrat J, Thilemann S, Saab AS, Kirchhoff F, Mobius W, Goebbels S, Nave KA et al: Neurotransmitter-triggered transfer of exosomes mediates oligodendrocyteneuron communication. PLoS biology 2013, 11(7):e1001604.

195. Antonucci F, Turola E, Riganti L, Caleo M, Gabrielli M, Perrotta C, Novellino L, Clementi E, Giussani $P$, Viani $P$ et al: Microvesicles released from microglia stimulate synaptic activity via enhanced sphingolipid metabolism. The EMBO journal 2012, 31(5):1231-1240.

196. Morel L, Regan M, Higashimori H, Ng SK, Esau C, Vidensky S, Rothstein J, Yang Y: Neuronal 
exosomal miRNA-dependent translational regulation of astroglial glutamate transporter GLT1. The Journal of biological chemistry 2013, 288(10):7105-7116.

197. Taylor AR, Robinson MB, Gifondorwa DJ, Tytell M, Milligan CE: Regulation of heat shock protein 70 release in astrocytes: role of signaling kinases. Developmental neurobiology 2007, 67(13):1815-1829.

198. Tytell M: Release of heat shock proteins (Hsps) and the effects of extracellular Hsps on neural cells and tissues. International journal of hyperthermia : the official journal of European Society for Hyperthermic Oncology, North American Hyperthermia Group 2005, 21(5):445-455.

199. Korkut C, Li Y, Koles K, Brewer C, Ashley J, Yoshihara M, Budnik V: Regulation of postsynaptic retrograde signaling by presynaptic exosome release. Neuron 2013, 77(6):1039-1046.

200. Ratajczak J, Miekus K, Kucia M, Zhang J, Reca R, Dvorak P, Ratajczak MZ: Embryonic stem cellderived microvesicles reprogram hematopoietic progenitors: evidence for horizontal transfer of mRNA and protein delivery. Leukemia 2006, 20(5):847-856.

201. Alvarez-Erviti L, Seow Y, Yin H, Betts C, Lakhal S, Wood MJ: Delivery of siRNA to the mouse brain by systemic injection of targeted exosomes. Nature biotechnology 2011, 29(4):341-345.

202. Grapp M, Wrede A, Schweizer M, Huwel S, Galla HJ, Snaidero N, Simons M, Buckers J, Low PS, Urlaub $\mathrm{H}$ et al: Choroid plexus transcytosis and exosome shuttling deliver folate into brain parenchyma. Nature communications 2013, 4:2123.

203. Rajendran L, Honsho M, Zahn TR, Keller P, Geiger KD, Verkade P, Simons K: Alzheimer's disease beta-amyloid peptides are released in association with exosomes. Proceedings of the National Academy of Sciences of the United States of America 2006, 103(30):11172-11177.

204. Saman S, Kim W, Raya M, Visnick Y, Miro S, Jackson B, McKee AC, Alvarez VE, Lee NC, Hall GF: Exosome-associated tau is secreted in tauopathy models and is selectively phosphorylated in cerebrospinal fluid in early Alzheimer disease. The Journal of biological chemistry 2012, 287(6):3842-3849.

205. Al-Nedawi K, Meehan B, Micallef J, Lhotak V, May L, Guha A, Rak J: Intercellular transfer of the oncogenic receptor EGFRvIII by microvesicles derived from tumour cells. Nature cell biology 2008, 10(5):619-624.

206. Li JY, Englund E, Holton JL, Soulet D, Hagell P, Lees AJ, Lashley T, Quinn NP, Rehncrona S, Bjorklund A et al: Lewy bodies in grafted neurons in subjects with Parkinson's disease suggest host-to-graft disease propagation. Nature medicine 2008, 14(5):501-503.

207. Kordower JH, Chu Y, Hauser RA, Freeman TB, Olanow CW: Lewy body-like pathology in long-term embryonic nigral transplants in Parkinson's disease. Nature medicine 2008, 14(5):504-506.

208. Schneider A, Simons M: Exosomes: vesicular carriers for intercellular communication in neurodegenerative disorders. Cell and tissue research 2013, 352(1):33-47.

209. Bellingham SA, Guo BB, Coleman BM, Hill AF: Exosomes: vehicles for the transfer of toxic proteins associated with neurodegenerative diseases? Frontiers in physiology 2012, 3:124.

210. Fevrier B, Vilette D, Laude H, Raposo G: Exosomes: a bubble ride for prions? Traffic 2005, 6(1):1017.

211. Ennaceur A, Meliani K: A new one-trial test for neurobiological studies of memory in rats. III. Spatial vs. non-spatial working memory. Behavioural brain research 1992, 51(1):83-92.

212. Langmead B, Salzberg SL: Fast gapped-read alignment with Bowtie 2. Nature methods 2012, 9(4):357-359.

213. Altschul SF, Gish W, Miller W, Myers EW, Lipman DJ: Basic local alignment search tool. Journal of molecular biology 1990, 215(3):403-410.

214. Anders S, Huber W: Differential expression analysis for sequence count data. Genome biology 2010, 11(10):R106. 
215. Supek F, Bosnjak M, Skunca N, Smuc T: REVIGO summarizes and visualizes long lists of gene ontology terms. PloS one 2011, 6(7):e21800.

216. Myers KM, Davis M: Mechanisms of fear extinction. Molecular psychiatry 2007, 12(2):120-150.

217. Subramanian A, Tamayo P, Mootha VK, Mukherjee S, Ebert BL, Gillette MA, Paulovich A, Pomeroy SL, Golub TR, Lander ES et al: Gene set enrichment analysis: a knowledge-based approach for interpreting genome-wide expression profiles. Proceedings of the National Academy of Sciences of the United States of America 2005, 102(43):15545-15550.

218. Wang X, Cairns MJ: Gene set enrichment analysis of RNA-Seq data: integrating differential expression and splicing. BMC bioinformatics 2013, 14 Suppl 5:S16.

219. The Gene Ontology project in 2008. Nucleic acids research 2008, 36(Database issue):D440-444.

220. Kanehisa M: A database for post-genome analysis. Trends in genetics : TIG 1997, 13(9):375-376.

221. Lu M, Shi B, Wang J, Cao Q, Cui Q: TAM: a method for enrichment and depletion analysis of a microRNA category in a list of microRNAs. BMC bioinformatics 2010, 11:419.

222. Radde R, Bolmont T, Kaeser SA, Coomaraswamy J, Lindau D, Stoltze L, Calhoun ME, Jaggi F, Wolburg $\mathrm{H}$, Gengler $\mathrm{S}$ et al: Abeta42-driven cerebral amyloidosis in transgenic mice reveals early and robust pathology. EMBO reports 2006, 7(9):940-946.

223. Govindarajan N, Rao P, Burkhardt S, Sananbenesi F, Schluter OM, Bradke F, Lu J, Fischer A: Reducing HDAC6 ameliorates cognitive deficits in a mouse model for Alzheimer's disease. EMBO molecular medicine 2013, 5(1):52-63.

224. Ermini FV, GrathwohI S, Radde R, Yamaguchi M, Staufenbiel M, Palmer TD, Jucker M: Neurogenesis and alterations of neural stem cells in mouse models of cerebral amyloidosis. The American journal of pathology 2008, 172(6):1520-1528.

225. Wegenast-Braun BM, Fulgencio Maisch A, Eicke D, Radde R, Herzig MC, Staufenbiel M, Jucker $M$, Calhoun ME: Independent effects of intra- and extracellular Abeta on learning-related gene expression. The American journal of pathology 2009, 175(1):271-282.

226. Zovoilis A, Agbemenyah HY, Agis-Balboa RC, Stilling RM, Edbauer D, Rao P, Farinelli L, Delalle I, Schmitt A, Falkai $P$ et al: microRNA-34c is a novel target to treat dementias. The EMBO journal 2011, 30(20):4299-4308.

227. Fisher $\mathrm{Y}$, Nemirovsky A, Baron R, Monsonego A: T cells specifically targeted to amyloid plaques enhance plaque clearance in a mouse model of Alzheimer's disease. PloS one 2010, 5(5):e10830.

228. Scholtzova H, Wadghiri YZ, Douadi M, Sigurdsson EM, Li YS, Quartermain D, Banerjee P, Wisniewski T: Memantine leads to behavioral improvement and amyloid reduction in Alzheimer's-diseasemodel transgenic mice shown as by micromagnetic resonance imaging. Journal of neuroscience research 2008, 86(12):2784-2791.

229. Kim J, Jiang H, Park S, Eltorai AE, Stewart FR, Yoon H, Basak JM, Finn MB, Holtzman DM: Haploinsufficiency of human APOE reduces amyloid deposition in a mouse model of amyloid-beta amyloidosis. The Journal of neuroscience : the official journal of the Society for Neuroscience 2011, 31(49):18007-18012.

230. Montarolo F, Parolisi R, Hoxha E, Boda E, Tempia F: Early enriched environment exposure protects 
spatial memory and accelerates amyloid plaque formation in APP(Swe)/PS1(L166P) mice. PloS one 2013, 8(7):e69381.

231. World Population Prospects: The 2012 Revision, Highlights and Advance Tables. United Nations, Department of Economic and Social Affairs, Population Division 2013.

232. Raftery AE, Li N, Sevcikova H, Gerland P, Heilig GK: Bayesian probabilistic population projections for all countries. Proceedings of the National Academy of Sciences of the United States of America 2012, 109(35):13915-13921.

233. Montesanto A, Dato S, Bellizzi D, Rose G, Passarino G: Epidemiological, genetic and epigenetic aspects of the research on healthy ageing and longevity. Immunity \& ageing : I \& A 2012, 9(1):6.

234. Nilsson LG: Memory function in normal aging. Acta neurologica Scandinavica Supplementum 2003, 179:7-13.

235. Hedden T, Gabrieli JD: Insights into the ageing mind: a view from cognitive neuroscience. Nature reviews Neuroscience 2004, 5(2):87-96.

236. Morse CK: Does variability increase with age? An archival study of cognitive measures. Psychology and aging 1993, 8(2):156-164.

237. Koivisto K, Reinikainen KJ, Hanninen T, Vanhanen M, Helkala EL, Mykkanen L, Laakso M, Pyorala K, Riekkinen PJ, Sr.: Prevalence of age-associated memory impairment in a randomly selected population from eastern Finland. Neurology 1995, 45(4):741-747.

238. Gallagher M, Nicolle MM: Animal models of normal aging: relationship between cognitive decline and markers in hippocampal circuitry. Behavioural brain research 1993, 57(2):155-162.

239. Aitken $\mathrm{DH}$, Meaney $\mathrm{MJ}$ : Temporally graded, age-related impairments in spatial memory in the rat. Neurobiology of aging 1989, 10(3):273-276.

240. Gage FH, Kelly PA, Bjorklund A: Regional changes in brain glucose metabolism reflect cognitive impairments in aged rats. The Journal of neuroscience : the official journal of the Society for Neuroscience 1984, 4(11):2856-2865.

241. Lu T, Pan Y, Kao SY, Li C, Kohane I, Chan J, Yankner BA: Gene regulation and DNA damage in the ageing human brain. Nature 2004, 429(6994):883-891.

242. Jiang $\mathrm{CH}$, Tsien JZ, Schultz PG, Hu Y: The effects of aging on gene expression in the hypothalamus and cortex of mice. Proceedings of the National Academy of Sciences of the United States of America 2001, 98(4):1930-1934.

243. Lee CK, Klopp RG, Weindruch R, Prolla TA: Gene expression profile of aging and its retardation by caloric restriction. Science 1999, 285(5432):1390-1393.

244. Peleg S, Sananbenesi F, Zovoilis A, Burkhardt S, Bahari-Javan S, Agis-Balboa RC, Cota P, Wittnam $\mathrm{JL}$, Gogol-Doering A, Opitz L et al: Altered histone acetylation is associated with age-dependent memory impairment in mice. Science 2010, 328(5979):753-756.

245. Freund J, Brandmaier AM, Lewejohann L, Kirste I, Kritzler M, Kruger A, Sachser N, Lindenberger U, Kempermann G: Emergence of individuality in genetically identical mice. Science 2013, 340(6133):756759. 
246. Logue SF, Paylor R, Wehner JM: Hippocampal lesions cause learning deficits in inbred mice in the Morris water maze and conditioned-fear task. Behavioral neuroscience 1997, 111(1):104-113.

247. LeDoux JE: Emotion circuits in the brain. Annual review of neuroscience 2000, 23:155-184.

248. Phillips RG, LeDoux JE: Differential contribution of amygdala and hippocampus to cued and contextual fear conditioning. Behavioral neuroscience 1992, 106(2):274-285.

249. Mumby DG, Pinel JP: Rhinal cortex lesions and object recognition in rats. Behavioral neuroscience 1994, 108(1):11-18.

250. O'Keefe J, Dostrovsky J: The hippocampus as a spatial map. Preliminary evidence from unit activity in the freely-moving rat. Brain research 1971, 34(1):171-175.

251. Kraus BJ, Robinson RJ, 2nd, White JA, Eichenbaum H, Hasselmo ME: Hippocampal "time cells": time versus path integration. Neuron 2013, 78(6):1090-1101.

252. Gallagher M, Burwell R, Burchinal M: Severity of spatial learning impairment in aging: development of a learning index for performance in the Morris water maze. Behavioral neuroscience 1993, 107(4):618626.

253. Merrill DA, Karim R, Darraq M, Chiba AA, Tuszynski MH: Hippocampal cell genesis does not correlate with spatial learning ability in aged rats. The Journal of comparative neurology 2003, 459(2):201207.

254. Le Jeune H, Cecyre D, Rowe W, Meaney MJ, Quirion R: Ionotropic glutamate receptor subtypes in the aged memory-impaired and unimpaired Long-Evans rat. Neuroscience 1996, 74(2):349-363.

255. Nyffeler M, Zhang WN, Feldon J, Knuesel I: Differential expression of PSD proteins in age-related spatial learning impairments. Neurobiology of aging 2007, 28(1):143-155.

256. Menard C, Quirion R: Group 1 metabotropic glutamate receptor function and its regulation of learning and memory in the aging brain. Frontiers in pharmacology 2012, 3:182.

257. Burger C, Lopez MC, Baker HV, Mandel RJ, Muzyczka N: Genome-wide analysis of aging and learning-related genes in the hippocampal dentate gyrus. Neurobiology of learning and memory 2008, 89(4):379-396.

258. Bizon JL, Lee HJ, Gallagher M: Neurogenesis in a rat model of age-related cognitive decline. Aging cell 2004, 3(4):227-234.

259. Drapeau E, Mayo W, Aurousseau C, Le Moal M, Piazza PV, Abrous DN: Spatial memory performances of aged rats in the water maze predict levels of hippocampal neurogenesis. Proceedings of the National Academy of Sciences of the United States of America 2003, 100(24):14385-14390.

260. Nyffeler M, Yee BK, Feldon J, Knuesel I: Abnormal differentiation of newborn granule cells in agerelated working memory impairments. Neurobiology of aging 2010, 31(11):1956-1974.

261. Stranahan AM: Putting age-associated changes in neurogenesis in their place. Neurobiology of aging 2010, 31(12):2179-2180.

262. Lazic SE: Relating hippocampal neurogenesis to behavior: the dangers of ignoring confounding variables. Neurobiology of aging 2010, 31(12):2169-2171; discussion 2172-2165.

263. Shamy JL, Baxter MG: Statistical considerations in the evaluation of potential contributions of 
individual neurobiological differences to cognitive performance. Commentary on Lazic (2010) "Relating hippocampal neurogenesis to behavior: the dangers of ignoring confounding variables". Neurobiology of aging 2010, 31(12):2176-2178.

264. Jin K, Peel AL, Mao XO, Xie L, Cottrell BA, Henshall DC, Greenberg DA: Increased hippocampal neurogenesis in Alzheimer's disease. Proceedings of the National Academy of Sciences of the United States of America 2004, 101(1):343-347.

265. Jin K, Galvan V, Xie L, Mao XO, Gorostiza OF, Bredesen DE, Greenberg DA: Enhanced neurogenesis in Alzheimer's disease transgenic (PDGF-APPSw,Ind) mice. Proceedings of the National Academy of Sciences of the United States of America 2004, 101(36):13363-13367.

266. Glisovic T, Bachorik JL, Yong J, Dreyfuss G: RNA-binding proteins and post-transcriptional gene regulation. FEBS letters 2008, 582(14):1977-1986.

267. Huber KM, Gallagher SM, Warren ST, Bear MF: Altered synaptic plasticity in a mouse model of fragile $X$ mental retardation. Proceedings of the National Academy of Sciences of the United States of America 2002, 99(11):7746-7750.

268. Antar LN, Dictenberg JB, Plociniak M, Afroz R, Bassell GJ: Localization of FMRP-associated mRNA granules and requirement of microtubules for activity-dependent trafficking in hippocampal neurons. Genes, brain, and behavior 2005, 4(6):350-359.

269. Antar LN, Afroz R, Dictenberg JB, Carroll RC, Bassell GJ: Metabotropic glutamate receptor activation regulates fragile $x$ mental retardation protein and FMR1 mRNA localization differentially in dendrites and at synapses. The Journal of neuroscience : the official journal of the Society for Neuroscience 2004, 24(11):2648-2655.

270. Napoli I, Mercaldo V, Boyl PP, Eleuteri B, Zalfa F, De Rubeis S, Di Marino D, Mohr E, Massimi M, Falconi $\mathrm{M}$ et al: The fragile $\mathrm{X}$ syndrome protein represses activity-dependent translation through CYFIP1, a new 4E-BP. Cell 2008, 134(6):1042-1054.

271. Zalfa F, Giorgi M, Primerano B, Moro A, Di Penta A, Reis S, Oostra B, Bagni C: The fragile X syndrome protein FMRP associates with $\mathrm{BC} 1 \mathrm{RNA}$ and regulates the translation of specific mRNAs at synapses. Cell 2003, 112(3):317-327.

272. Ronesi JA, Huber KM: Metabotropic glutamate receptors and fragile $x$ mental retardation protein: partners in translational regulation at the synapse. Science signaling 2008, 1(5):pe6.

273. Lebeau G, Miller LC, Tartas M, McAdam R, Laplante I, Badeaux F, DesGroseillers L, Sossin WS, Lacaille JC: Staufen 2 regulates $m$ GluR long-term depression and Map1b mRNA distribution in hippocampal neurons. Learn Mem 2011, 18(5):314-326.

274. Miki T, Takano K, Yoneda Y: The role of mammalian Staufen on mRNA traffic: a view from its nucleocytoplasmic shuttling function. Cell structure and function 2005, 30(2):51-56.

275. Hoeffer CA, Klann E: mTOR signaling: at the crossroads of plasticity, memory and disease. Trends in neurosciences 2010, 33(2):67-75.

276. Klann E, Dever TE: Biochemical mechanisms for translational regulation in synaptic plasticity. Nature reviews Neuroscience 2004, 5(12):931-942.

277. Tang SJ, Reis G, Kang H, Gingras AC, Sonenberg N, Schuman EM: A rapamycin-sensitive signaling 
pathway contributes to long-term synaptic plasticity in the hippocampus. Proceedings of the National Academy of Sciences of the United States of America 2002, 99(1):467-472.

278. Huber KM, Roder JC, Bear MF: Chemical induction of mGluR5- and protein synthesis--dependent long-term depression in hippocampal area CA1. Journal of neurophysiology 2001, 86(1):321-325.

279. Kolosova NG, Vitovtov AO, Muraleva NA, Akulov AE, Stefanova NA, Blagosklonny MV: Rapamycin suppresses brain aging in senescence-accelerated OXYS rats. Aging 2013, 5(6):474-484.

280. Harrison DE, Strong R, Sharp ZD, Nelson JF, Astle CM, Flurkey K, Nadon NL, Wilkinson JE, Frenkel $\mathrm{K}$, Carter CS et al: Rapamycin fed late in life extends lifespan in genetically heterogeneous mice. Nature 2009, 460(7253):392-395.

281. Lang UE, Heger J, Willbring M, Domula M, Matschke K, Tugtekin SM: Immunosuppression using the mammalian target of rapamycin (mTOR) inhibitor everolimus: pilot study shows significant cognitive and affective improvement. Transplantation proceedings 2009, 41(10):4285-4288.

282. Pirola L, Bonnafous S, Johnston AM, Chaussade C, Portis F, Van Obberghen E: Phosphoinositide 3-kinase-mediated reduction of insulin receptor substrate-1/2 protein expression via different mechanisms contributes to the insulin-induced desensitization of its signaling pathways in L6 muscle cells. The Journal of biological chemistry 2003, 278(18):15641-15651.

283. Lucas DR, Newhouse JP: The toxic effect of sodium L-glutamate on the inner layers of the retina. AMA archives of ophthalmology 1957, 58(2):193-201.

284. Olney JW: Brain lesions, obesity, and other disturbances in mice treated with monosodium glutamate. Science 1969, 164(3880):719-721.

285. Pitt D, Werner P, Raine CS: Glutamate excitotoxicity in a model of multiple sclerosis. Nature medicine 2000, 6(1):67-70.

286. Camacho A, Massieu L: Role of glutamate transporters in the clearance and release of glutamate during ischemia and its relation to neuronal death. Archives of medical research 2006, 37(1):11-18.

287. Ankarcrona M, Dypbukt JM, Bonfoco E, Zhivotovsky B, Orrenius S, Lipton SA, Nicotera P: Glutamate-induced neuronal death: a succession of necrosis or apoptosis depending on mitochondrial function. Neuron 1995, 15(4):961-973.

288. Hynd MR, Scott HL, Dodd PR: Glutamate-mediated excitotoxicity and neurodegeneration in Alzheimer's disease. Neurochemistry international 2004, 45(5):583-595.

289. Olney JW, Wozniak DF, Farber NB: Excitotoxic neurodegeneration in Alzheimer disease. New hypothesis and new therapeutic strategies. Archives of neurology 1997, 54(10):1234-1240.

290. Rodriguez MC, Obeso JA, Olanow CW: Subthalamic nucleus-mediated excitotoxicity in Parkinson's disease: a target for neuroprotection. Annals of neurology 1998, 44(3 Suppl 1):S175-188.

291. Hulsebosch CE, Hains BC, Crown ED, Carlton SM: Mechanisms of chronic central neuropathic pain after spinal cord injury. Brain research reviews 2009, 60(1):202-213.

292. Leigh PN, Meldrum BS: Excitotoxicity in ALS. Neurology 1996, 47(6 Suppl 4):S221-227.

293. Lipton SA: Paradigm shift in neuroprotection by NMDA receptor blockade: memantine and beyond. Nature reviews Drug discovery 2006, 5(2):160-170. 
294. Lipton SA: The molecular basis of memantine action in Alzheimer's disease and other neurologic disorders: low-affinity, uncompetitive antagonism. Current Alzheimer research 2005, 2(2):155-165.

295. Schonrock N, Ke YD, Humphreys D, Staufenbiel M, Ittner LM, Preiss T, Gotz J: Neuronal microRNA deregulation in response to Alzheimer's disease amyloid-beta. PloS one 2010, 5(6):e11070.

296. Wang X, Liu P, Zhu H, Xu Y, Ma C, Dai X, Huang L, Liu Y, Zhang L, Qin C: miR-34a, a microRNA up-regulated in a double transgenic mouse model of Alzheimer's disease, inhibits bcl2 translation. Brain research bulletin 2009, 80(4-5):268-273.

297. Patel N, Hoang D, Miller N, Ansaloni S, Huang Q, Rogers JT, Lee JC, Saunders AJ: MicroRNAs can regulate human APP levels. Molecular neurodegeneration 2008, 3:10.

298. Long JM, Lahiri DK: MicroRNA-101 downregulates Alzheimer's amyloid-beta precursor protein levels in human cell cultures and is differentially expressed. Biochemical and biophysical research communications 2011, 404(4):889-895.

299. Vilardo E, Barbato C, Ciotti M, Cogoni C, Ruberti F: MicroRNA-101 regulates amyloid precursor protein expression in hippocampal neurons. The Journal of biological chemistry 2010, 285(24):1834418351.

300. Hebert SS, Horre K, Nicolai L, Bergmans B, Papadopoulou AS, Delacourte A, De Strooper B: MicroRNA regulation of Alzheimer's Amyloid precursor protein expression. Neurobiology of disease 2009, 33(3):422-428.

301. Delay C, Calon F, Mathews P, Hebert SS: Alzheimer-specific variants in the 3'UTR of Amyloid precursor protein affect microRNA function. Molecular neurodegeneration 2011, 6:70.

302. Hebert SS, Horre K, Nicolai L, Papadopoulou AS, Mandemakers W, Silahtaroglu AN, Kauppinen S, Delacourte A, De Strooper B: Loss of microRNA cluster miR-29a/b-1 in sporadic Alzheimer's disease correlates with increased BACE1/beta-secretase expression. Proceedings of the National Academy of Sciences of the United States of America 2008, 105(17):6415-6420.

303. Schonrock N, Matamales M, Ittner LM, Gotz J: MicroRNA networks surrounding APP and amyloidbeta metabolism--implications for Alzheimer's disease. Experimental neurology 2012, 235(2):447-454.

304. Cogswell JP, Ward J, Taylor IA, Waters M, Shi Y, Cannon B, Kelnar K, Kemppainen J, Brown $D$, Chen $C$ et al: Identification of miRNA changes in Alzheimer's disease brain and CSF yields putative biomarkers and insights into disease pathways. Journal of Alzheimer's disease : JAD 2008, 14(1):27-41.

305. Nunez-Iglesias J, Liu CC, Morgan TE, Finch CE, Zhou XJ: Joint genome-wide profiling of miRNA and mRNA expression in Alzheimer's disease cortex reveals altered miRNA regulation. PloS one 2010, $5(2): \mathrm{e} 8898$.

306. Lau P, Bossers K, Janky R, Salta E, Frigerio CS, Barbash S, Rothman R, Sierksma AS, Thathiah A, Greenberg D et al: Alteration of the microRNA network during the progression of Alzheimer's disease. EMBO molecular medicine 2013, 5(10):1613-1634.

307. Shioya M, Obayashi S, Tabunoki H, Arima K, Saito Y, Ishida T, Satoh J: Aberrant microRNA expression in the brains of neurodegenerative diseases: miR-29a decreased in Alzheimer disease brains targets neurone navigator 3. Neuropathology and applied neurobiology 2010, 36(4):320-330.

308. Wang WX, Rajeev BW, Stromberg AJ, Ren N, Tang G, Huang Q, Rigoutsos I, Nelson PT: The 
expression of microRNA miR-107 decreases early in Alzheimer's disease and may accelerate disease progression through regulation of beta-site amyloid precursor protein-cleaving enzyme 1 . The Journal of neuroscience : the official journal of the Society for Neuroscience 2008, 28(5):1213-1223.

309. Nelson PT, Wang WX: MiR-107 is reduced in Alzheimer's disease brain neocortex: validation study. Journal of Alzheimer's disease : JAD 2010, 21(1):75-79.

310. Lukiw WJ: Micro-RNA speciation in fetal, adult and Alzheimer's disease hippocampus. Neuroreport 2007, 18(3):297-300.

311. Delay C, Mandemakers W, Hebert SS: MicroRNAs in Alzheimer's disease. Neurobiology of disease 2012, 46(2):285-290.

312. Brenner S, Jacob F, Meselson M: An unstable intermediate carrying information from genes to ribosomes for protein synthesis. Nature 1961, 190:576-581.

313. Chomczynski P, Sacchi N: Single-step method of RNA isolation by acid guanidinium thiocyanatephenol-chloroform extraction. Analytical biochemistry 1987, 162(1):156-159.

314. Puissant C, Houdebine LM: An improvement of the single-step method of RNA isolation by acid guanidinium thiocyanate-phenol-chloroform extraction. BioTechniques 1990, 8(2):148-149.

315. Mitchell PS, Parkin RK, Kroh EM, Fritz BR, Wyman SK, Pogosova-Agadjanyan EL, Peterson A, Noteboom J, O'Briant KC, Allen A et al: Circulating microRNAs as stable blood-based markers for cancer detection. Proceedings of the National Academy of Sciences of the United States of America 2008, 105(30):10513-10518.

316. Noerholm M, Balaj L, Limperg T, Salehi A, Zhu LD, Hochberg FH, Breakefield XO, Carter BS, Skog $\mathrm{J}$ : RNA expression patterns in serum microvesicles from patients with glioblastoma multiforme and controls. BMC cancer 2012, 12:22.

317. Chen WW, Balaj L, Liau LM, Samuels ML, Kotsopoulos SK, Maguire CA, Loguidice L, Soto H, Garrett M, Zhu LD et al: BEAMing and Droplet Digital PCR Analysis of Mutant IDH1 mRNA in Glioma Patient Serum and Cerebrospinal Fluid Extracellular Vesicles. Molecular therapy Nucleic acids 2013, 2:e109.

318. Crescitelli R, Lasser C, Szabo TG, Kittel A, Eldh M, Dianzani I, Buzas El, Lotvall J: Distinct RNA profiles in subpopulations of extracellular vesicles: apoptotic bodies, microvesicles and exosomes. Journal of extracellular vesicles 2013, 2.

319. Burgos KL, Javaherian A, Bomprezzi R, Ghaffari L, Rhodes S, Courtright A, Tembe W, Kim S, Metpally R, Van Keuren-Jensen K: Identification of extracellular miRNA in human cerebrospinal fluid by next-generation sequencing. RNA 2013, 19(5):712-722.

320. Eldh M, Lotvall J, Malmhall C, Ekstrom K: Importance of RNA isolation methods for analysis of exosomal RNA: evaluation of different methods. Molecular immunology 2012, 50(4):278-286.

321. McAlexander MA, Phillips MJ, Witwer KW: Comparison of Methods for miRNA Extraction from Plasma and Quantitative Recovery of RNA from Cerebrospinal Fluid. Frontiers in genetics 2013, 4:83.

322. Muller M, Kuiperij HB, Claassen JA, Kusters B, Verbeek MM: MicroRNAs in Alzheimer's disease: differential expression in hippocampus and cell-free cerebrospinal fluid. Neurobiology of aging 2014, 35(1):152-158.

323. Taylor DD, Gercel-Taylor C: MicroRNA signatures of tumor-derived exosomes as diagnostic 
biomarkers of ovarian cancer. Gynecologic oncology 2008, 110(1):13-21.

324. Valadi H, Ekstrom K, Bossios A, Sjostrand M, Lee JJ, Lotvall JO: Exosome-mediated transfer of mRNAs and microRNAs is a novel mechanism of genetic exchange between cells. Nature cell biology 2007, 9(6):654-659.

325. Lasser C, Alikhani VS, Ekstrom K, Eldh M, Paredes PT, Bossios A, Sjostrand M, Gabrielsson S, Lotvall J, Valadi H: Human saliva, plasma and breast milk exosomes contain RNA: uptake by macrophages. Journal of translational medicine 2011, 9:9.

326. Palanisamy V, Sharma S, Deshpande A, Zhou H, Gimzewski J, Wong DT: Nanostructural and transcriptomic analyses of human saliva derived exosomes. PloS one 2010, 5(1):e8577.

327. Nolte-'t Hoen EN, Buermans HP, Waasdorp M, Stoorvogel W, Wauben MH, t Hoen PA: Deep sequencing of RNA from immune cell-derived vesicles uncovers the selective incorporation of small noncoding RNA biotypes with potential regulatory functions. Nucleic acids research 2012, 40(18):9272-9285.

328. Bellingham SA, Coleman BM, Hill AF: Small RNA deep sequencing reveals a distinct miRNA signature released in exosomes from prion-infected neuronal cells. Nucleic acids research 2012, 40(21):10937-10949.

329. Ogawa Y, Taketomi Y, Murakami M, Tsujimoto M, Yanoshita R: Small RNA transcriptomes of two types of exosomes in human whole saliva determined by next generation sequencing. Biological \& pharmaceutical bulletin 2013, 36(1):66-75.

330. Diehl P, Fricke A, Sander L, Stamm J, Bassler N, Htun N, Ziemann M, Helbing T, El-Osta A, Jowett JB et al: Microparticles: major transport vehicles for distinct microRNAs in circulation. Cardiovascular research 2012, 93(4):633-644.

331. Balaj L, Lessard R, Dai L, Cho YJ, Pomeroy SL, Breakefield XO, Skog J: Tumour microvesicles contain retrotransposon elements and amplified oncogene sequences. Nature communications 2011, 2:180.

332. Alexandrov PN, Dua P, Hill JM, Bhattacharjee S, Zhao Y, Lukiw WJ: microRNA (miRNA) speciation in Alzheimer's disease (AD) cerebrospinal fluid (CSF) and extracellular fluid (ECF). International journal of biochemistry and molecular biology 2012, 3(4):365-373.

333. Clemson CM, Hutchinson JN, Sara SA, Ensminger AW, Fox AH, Chess A, Lawrence JB: An architectural role for a nuclear noncoding RNA: NEAT1 RNA is essential for the structure of paraspeckles. Molecular cell 2009, 33(6):717-726.

334. Bond CS, Fox AH: Paraspeckles: nuclear bodies built on long noncoding RNA. The Journal of cell biology 2009, 186(5):637-644.

335. Stilling RM; 2013.

336. Bai B, Hales CM, Chen PC, Gozal Y, Dammer EB, Fritz JJ, Wang X, Xia Q, Duong DM, Street C et al: U1 small nuclear ribonucleoprotein complex and RNA splicing alterations in Alzheimer's disease. Proceedings of the National Academy of Sciences of the United States of America 2013, 110(41):1656216567. 
References 



\section{Appendix 1: Notes on Gene set enrichment analysis}

Gene set enrichment analysis (GSEA), is a tool that was developed to help study the biological significance to the large amounts of gene expression data generated by genone-wide studies such as microarrays [1]. This approach relies on prior biological knowledge to search for sets of genes whose expression is increased or decreased in the given gene list. Some examples of the 'sets' of genes used in GSEA are functionally related genes within Gene Ontology (GO) terms, genes in a pathway such as a KEGG pathway, genes within a defined chromosomal proximity, and genes with common transcription factor or miRNA binding sites. The main steps involved in GSEA are as follows : A list of genes weighted or ranked by expression change(fold change or $\mathrm{P}$ value) is generated based on user input. An enrichment score is calculated for the gene sets of interest and statistical significance is estimated and adjusted for multiple testing. Thus the analysis is focused on assigning significance values to a related group of genes rather than to individual transcripts.

One advantage of GSEA over a conventional differential expression analysis with a $p$-value cutoff is that GSEA has the ability to identify subtle or low-volume changes that occur across a set of related genes. This is particularly useful in identifying key regulatory networks in a situation where several genes in a pathway or functional category show small changes, but only a handful of them pass the arbitratry significance threshold.

In the work described in this thesis, as well as in other published studies, the criteria used to select the input list of genes are not very stringent and the resulting list is often large. This list may include all genes which change significantly with an unadjusted $p$ value, or even the entire list of expressed genes, weighted by fold change or $P$ value. The correction for multiple testing is then performed at the level of the gene set, rather than individual genes. The standard implementation of GSEA, available from the Broad Institute was used in this work, but many other methods to implement GSEA and to use custom gene sets for analysis also exist. Since its forst description in 2005 [1], GSEA has been used in a large number of studies and is a widely accepted method of analyzing genome-wide expression data.

1. Subramanian A, Tamayo P, Mootha VK, Mukherjee S, Ebert BL, Gillette MA, Paulovich A, Pomeroy SL, Golub TR, Lander ES et al: Gene set enrichment analysis: a knowledge-based approach for interpreting genome-wide expression profiles. Proceedings of the National Academy of Sciences of the United States of America 2005, 102(43):15545-15550. 



\section{Appendix 2: Notes on the Random Forest machine learning algorithm and the Gini Index}

The Random Forest algorithm is a machine learning method for classification of data (1). It works by classifying the samples using a large number of random decision trees, with one or more features used to perform the split at each node. This algorithm was chosen for the task because of its high accuracy and low likelihood of 'overfitting'. Further, this method provides an inbuilt means, through bootstrapping to conduct internal cross-validation and estimate error rates (out-of-bag error rates) and to calculate the relative importance of the variables used.

Note on interpretation of Decrease in Gini Index

The 'Decrease in Gini Index' is the method of choice for rapid estimation of the relative importance of variables or their contribution to effect being studied in a random forest algorithm (2). The Gini Index is a widely used statistical measure of inequality (3). In each tree of the constructed forest, when the given variable is used to split samples, the Gini Index or inequality in the resulting groups is measured. An important or useful variable would lead to an accurate splitting and thus the new group formed would be more homogenous. This would lead to a decrease in Gini index, which is measured by the algorithm and averaged over all the trees.

1. Breiman, Leo (2001). "Random Forests". Machine Learning 45 (1): 5-32. 1.

2. A. Liaw and M. Wiener (2002). Classification and Regression by randomForest. R News 2(3), 18--22

3. Gini, C. (1912). "Italian: Variabilità e mutabilità" 'Variability and Mutability', C. Cuppini, Bologna, 156 pages. Reprinted in Memorie di metodologica statistica (Ed. Pizetti E, Salvemini, T). Rome: Libreria Eredi Virgilio Veschi (1955) 